\section{Pacific Northwest}

National Laboratory

Operated by Battelle for the

U.S. Department of Energy

\title{
A Study of Past, Present, and Future Radiation Dose Pathways from Hanford Site Effluents
}

\author{
E. J. Antonio \\ K. Rhoads \\ L. H. Staven
}

March 2002

Prepared for the U.S. Department of Energy under Contract DE-AC06-76RL01830 


\title{
DISCLAIMER
}

This report was prepared as an account of work sponsored by an agency of the United States Government. Neither the United States Government nor any agency thereof, nor Battelle Memorial Institute, nor any of their employees, makes any warranty, express or implied, or assumes any legal liability or responsibility for the accuracy, completeness, or usefulness of any information, apparatus, product, or process disclosed, or represents that its use would not infringe privately owned rights. Reference herein to any specific commercial product, process, or service by trade name, trademark, manufacturer, or otherwise does not necessarily constitute or imply its endorsement, recommendation, or favoring by the United States Government or any agency thereof, or Battelle Memorial Institute. The views and opinions of authors expressed herein do not necessarily state or reflect those of the United States Government or any agency thereof.

\author{
PACIFIC NORTHWEST NATIONAL LABORATORY \\ operated by \\ BATTELLE \\ for the \\ UNITED STATES DEPARTMENT OF ENERGY \\ under Contract DE-ACO6-76RL0183O
}

Printed in the United States of America

\author{
Available to DOE and DOE contractors from the \\ Office of Scientific and Technical Information, \\ P.O. Box 62, Oak Ridge, TN 37831-0062; \\ ph: (865) 576-8401 \\ fax: (865) 576-5728 \\ email: reports@adonis.osti.gov
}

\footnotetext{
Available to the public from the National Technical Information Service, U.S. Department of Commerce, 5285 Port Royal Rd., Springfield, VA 22161 ph: (800) 553-6847 fax: (703) 605-6900

email: orders@ntis.fedworld.gov

online ordering: http://www.ntis.gov/ordering.htm
}

$\Delta$ This document was printed on recycled paper. 


\title{
A Study of Past, Present, and Future Radiation Dose Pathways from Hanford Site Effluents
}

\author{
E. J. Antonio \\ K. Rhoads \\ L. H. Staven
}

March 2002

Prepared for

the U.S. Department of Energy

under Contract DE-AC06-76RL01830

Pacific Northwest National Laboratory

Richland, Washington 99352 


\section{Summary}

This report also discusses current Washington State and Federal radiological water quality standards as well as air emissions standards and methods used at the Hanford Site to demonstrate compliance with these standards. Historically, the maximally exposed individual (MEI) has been a hypothetical farmer situated at the site perimeter who is postulated to maximize exposures from Hanford effluents released to the atmosphere or to the Columbia River. Three MEI locations have historically been used: a) Ringold, to maximize the impact from the 200 Area atmospheric releases, b) Sagemoor, to maximize impacts from 300 Area atmospheric releases, and c) Riverview, to maximize impacts from radionuclides released to the Columbia River. The report presents detailed pathway analyses for 1993-2001. The future location of the MEI will be affected by changes in the Hanford mission and activities (from past nuclear material production to environmental cleanup), as well as by new research projects, the leasing of property within the Hanford site boundary for private uses, and release of Hanford property for unrestricted use.

Recently, there has been an effort to evaluate radiological doses to onsite persons and to those who do not work for the U.S. Department of Energy or one of its contractors. The report describes methods for evaluating doses to an onsite MEI and discusses circumstances that may result in a need for additional air and external radiation surveillance. 


\section{Contents}

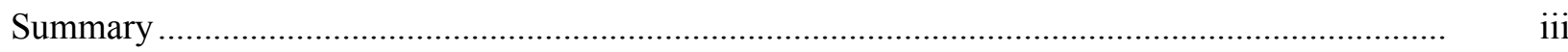

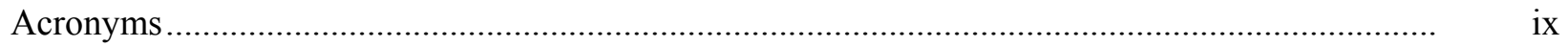

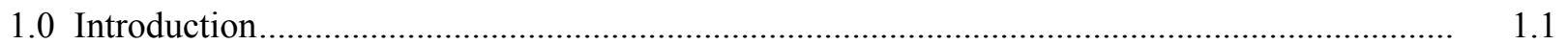

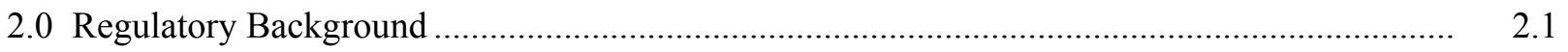

2.1 DOE Requirements under "Radiation Protection of the Public and Environment"

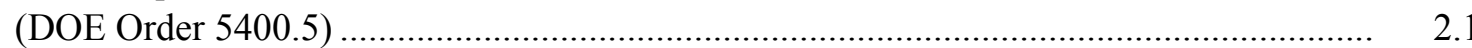

2.2 Federal and State Water Quality Standards .................................................................. 2.2

2.3 Federal and State Radionuclide Air Emissions Standards ...............................................

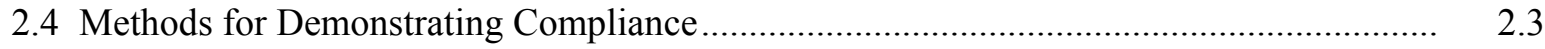

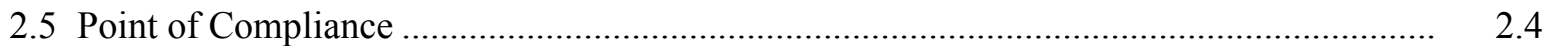

2.6 Permitting New or Modified Sources of Radionuclide Emissions ..................................... 2.4

3.0 Results of Recent Dose Assessments for Members of the Public From Hanford Site

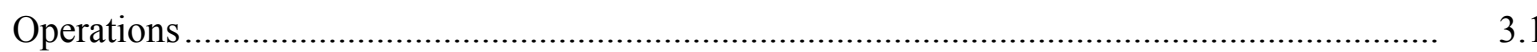

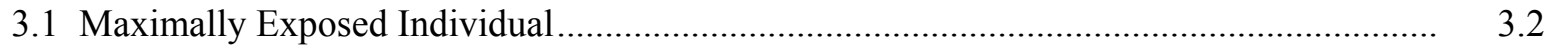

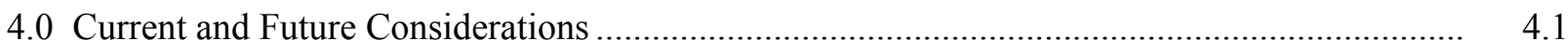

4.1 Changing Hanford Site Missions and Activities......................................................... 4.1

4.2 Environmental Restoration Activities................................................................................

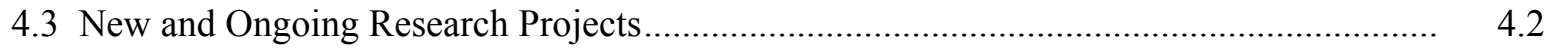

4.4 Leasing and Privatization of Hanford Site Facilities and Activities ................................. 4.2

4.5 Release of Hanford Lands for Public Use....................................................................

4.6 Community Growth and Development .......................................................................

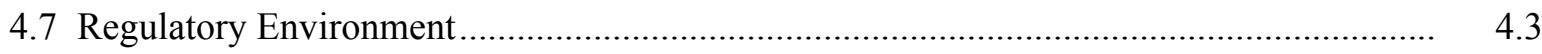


5.0 Potential Consequences for Exposure, Dose, and Risk Assessment..................................... 5.1

5.1 Evaluation of Dose to the Offsite Resident......................................................................

5.2 Methods for Evaluating Dose to an Onsite Maximally Exposed Individual ..................... $\quad 5.2$

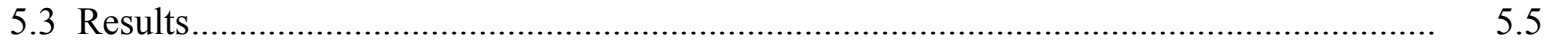

6.0 Proposed Changes to Methods for Evaluating Exposure, Dose, and Risk ............................ 6.1

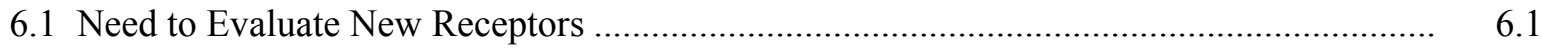

6.2 Need for Additional Radiological Monitoring - Air or External ................................... 6.1

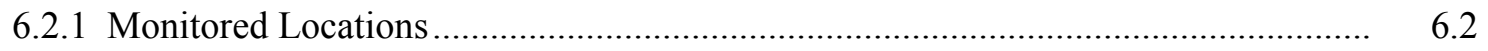

6.2.2 Monitored Radionuclides ............................................................................ 6.2

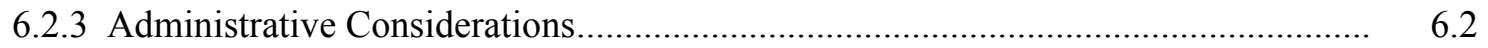

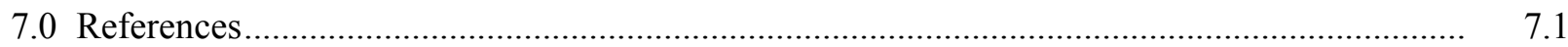

Appendix A - Parameters Used in Dose Calculations ….................................................... A.1

Appendix B - Doses to Maximally Exposed Individual, 1993-2000 _......................................... B. 1

Appendix C - Radionuclide Release Quantities, 1993-2000 ............................................... C.1

Appendix D - Standard Assumptions Used for Hanford Site Dose Assessments ............................ D.1

Appendix E - Comparison of Onsite Worker to Offsite Resident Doses for 200 W Area............... E.1

Appendix F - Comparison of Onsite Worker to Offsite Resident Doses for All Areas ................... F. F.1

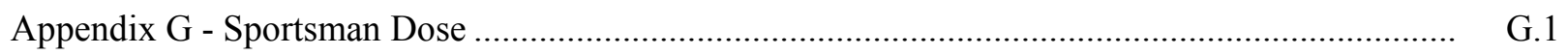




\section{Figures}

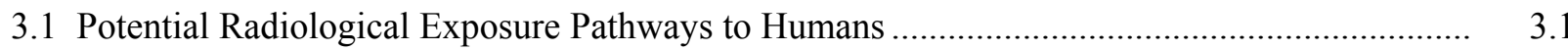

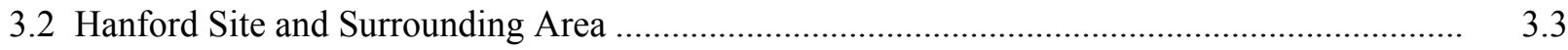

3.3 Calculated Radiological Doses to the MEI, $1.5 \mathrm{~km}$ East of the 300 Area, 1993 through 1999, 2000 at Riverview - Line at 5 mrem is annual variation in natural background

5.1 Stack Release Points and Onsite Receptors in 300 Area

\section{Tables}

3.1 Potential Radiological Doses to Hypothetical Maximally Exposed Individual

5.1 Distances and Directions From Various Release Points to Potential Onsite Workers and Offsite Residents Being Evaluated for Potential MEI Status for Annual Compliance Reporting Purposes

5.2 200-West Area, Dose per Unit Release for Onsite Worker at LIGO and Offsite Resident

5.3 Simulation Results of Radionuclides in CAP88 Library and Locations for which the Dose Rates per Unit Release to Onsite Worker Exceeds Dose to Offsite Resident. 


\section{Acronyms}

$\begin{array}{ll}\text { ALE } & \text { Fitzner-Eberhardt Arid Lands Ecology Reserve } \\ \text { DOE } & \text { U.S. Department of Energy } \\ \text { EDE } & \text { effective dose equivalent } \\ \text { EPA } & \text { U.S. Environmental Protection Agency } \\ \text { LIGO } & \text { Laser Interferometer Gravitational Wave Observatory } \\ \text { MEI } & \text { maximally exposed individual } \\ \text { NESHAP } & \text { National Emission Standards for Hazardous Air Pollutants } \\ \text { NPDES } & \text { National Pollutant Discharge Elimination System } \\ \text { NRC } & \text { U.S. Nuclear Regulatory Commission } \\ \text { PTE } & \text { potential to emit } \\ \text { TEDE } & \text { total effective dose equivalent } \\ \text { TLD } & \text { thermoluminescent dosimeter } \\ \text { WAC } & \text { Washington Administrative Code } \\ \text { WADOE } & \text { Washington State Department of Ecology } \\ \text { WADOH } & \text { Washington State Department of Health } \\ \text { WSU } & \text { Washington State University }\end{array}$

\section{Useful Conversions}

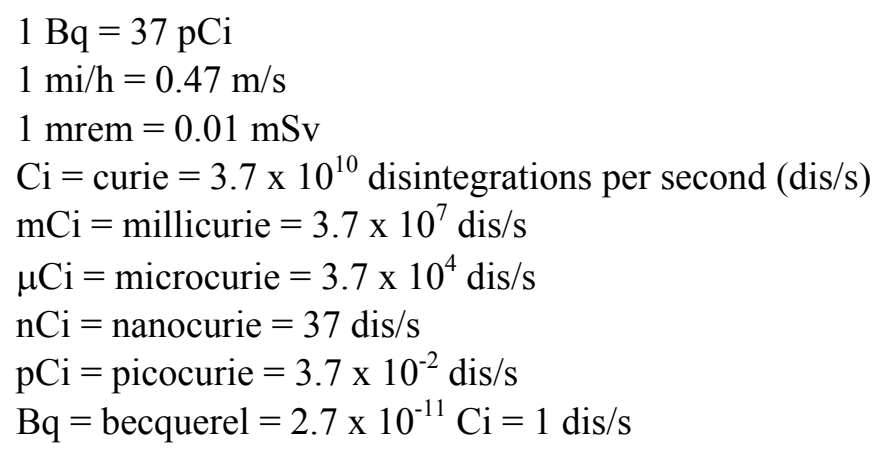




\subsection{Introduction}

This study was conducted by staff of the Pacific Northwest National Laboratory ${ }^{(a)}$ for the U.S. Department of Energy (DOE) to determine to what extent additional dose assessments should be made to comply with radiation protection standards established by the U.S. Environmental Protection Agency (EPA), Washington State Department of Health (WADOH), and DOE Orders. Members of the general public are now working on previously restricted portions of the Hanford Site in Washington State. In the recent past, compliance assessments consisted of determination of the offsite maximally exposed individual (offsite MEI) total effective dose equivalent (TEDE, hereafter referred to as "dose") via airborne pathways for compliance with EPA regulations, and total dose to the maximally exposed offsite individual from all pathways for determination of compliance with DOE Orders. In the following sections, the regulatory climate is presented as well as recent calculations of offsite and onsite MEI dose for the year 2000. Recommendations for future assessments are also included. Future activities that may require re-assessment of MEI locations or environmental surveillance are also identified.

(a) The Pacific Northwest National Laboratory is operated by Battelle Memorial Institute for the U.S. Department of Energy under Contract De-AC06-76RLO 1830. 


\subsection{Regulatory Background}

Limits established by DOE as well as standards of other Federal and state agencies regulate radioactive materials in effluents from DOE facilities. These limits and standards establish the maximum radiation dose received by a member of the public from DOE operations. The primary limits are described in DOE Order 5400.5; however, other regulations also apply to public radiation exposures by specific routes or pathways. Regulations that govern radiological dose to members of the public are summarized in the following sections.

\subsection{DOE Requirements under "Radiation Protection of the Public and Environment" (DOE Order 5400.5)}

U.S. Department of Energy facilities are required to demonstrate compliance with DOE Order 5400.5, which establishes radiation dose limits for members of the public from DOE operations. This Order also requires compliance with EPA regulations for exposure of members of the public to airborne radioactive effluents from DOE facilities, and specifies limits consistent with EPA regulations for public exposures via the drinking water pathway. Pertinent sections of the DOE Order are as follows:

- Section (Introduction) 10.d.(8), Definitions

"Public Dose means the dose received by member(s) of the public from exposure to radiation and to the radioactive material released by a DOE facility or operation, whether the exposure is within a DOE site boundary or offsite."

"Members of the Public means persons who are not occupationally associated with the DOE facility or operations, i.e., persons whose assigned occupational duties do not require them to enter the DOE site."

- Section II.1.a., Public Dose Limit - All Exposure Modes, All DOE Sources of Radiation

"Except as provided by II.1.a (4)(Exceptions), the exposure of members of the public to radiation sources as a consequence of all routine DOE activities shall not cause, in a year, an EDE (effective dose equipment) greater than 100 mrem."

- Section II.1.b., Public Dose Limit - Air Emissions Only, All DOE Sources of Radionuclides -

"To the extent required by Clean Air Act (CAA), the exposure of members of the public to radioactive materials released to the atmosphere as a consequence of routine DOE activities shall not cause members of the public to receive in a year, an EDE greater than $10 \mathrm{mrem} . "$

- Section II.1.d., Public Dose Limit - Drinking Water Pathway Only, All DOE Sources of Radionuclides 
"It is the policy of DOE to provide a level of protection for persons consuming water from a public drinking water supply operated by the DOE, either directly or through a DOE contractor, that is equivalent to that provided to the public by the public community drinking water standards of $40 \mathrm{CFR}$ Part 141. These systems shall not cause persons consuming the water to receive an effective dose equivalent greater than $4 \mathrm{mrem}(0.04 \mathrm{mSv})$ in a year. Combined radium-226 and radium-228 shall not exceed $5 \times 10^{-9} \mathrm{microCi} / \mathrm{ml}$ and gross alpha activity (including radium-226 but excluding radon and uranium) shall not exceed $1.5 \times 10^{-8} \mathrm{microCi} / \mathrm{ml} . "$

- Section II.6., Demonstration of Compliance with the Dose Limits

"Compliance with the dose limits of this Order shall be demonstrated by documentation of an appropriate combination of measurements and calculations to evaluate potential doses and the results of the evaluations."

\subsection{Federal and State Water Quality Standards}

U.S. Department of Energy facilities are also required to limit radionuclide concentrations in liquid effluents below levels that would cause public drinking water systems to exceed standards specified in 40 CFR Part 141. Currently, this standard applies to effluents discharged to the Columbia River because there are no DOE-operated public drinking water systems that utilize groundwater beneath the Hanford Site. However, Hanford has established waste-acceptance criteria (Fluor Hanford 2001) that limit radionuclide content and establish waste-disposal practices intended to protect groundwater to the same degree as public drinking water systems. These requirements are implemented through DOE Order 435.1, "Radioactive Waste Management," and site-specific performance assessments for radioactive waste disposal facilities. Corresponding Washington State regulations appear in Washington Administrative Code (WAC) 173-200, "Water Quality Standards for Ground Waters of the State of Washington," and in WAC 246-290, "Public Water Supplies." These WACs parallel Federal drinking water standards set forth in 40 CFR Part 141.

Liquid effluents are discharged from facilities in all areas of the Hanford Site. Effluents that normally or potentially contain radionuclides include cooling water, steam condensates, process condensates, and wastewater from laboratories and chemical sewers. In the 400 Area, the Fast Flux Test Facility is cooled with liquid sodium, using no water to cool the reactor or to moderate neutrons in the reactor core. Therefore, there are no radioactive liquid effluents released from the 400 Area. In December 1994, the 300 Area Treated Effluent Disposal Facility began receiving and treating wastewater. The treated liquid effluent is monitored and discharged through an outfall to the Columbia River under a National Pollutant Discharge Elimination System (NPDES) permit. Therefore, since 1995 no radioactive liquid discharges from the 300 Area have been reported.

The 200 Areas discharge liquid effluents containing tritium to the ground, which goes to the stateapproved land disposal site. DOE has, as a policy, ceased releasing liquid effluents directly to the ground. Liquid effluents are currently treated to meet acceptance criteria and then sent to the state-approved land disposal site. 
Liquid effluents from the 100-Areas seep into the Columbia River, the sources of which include secondary cooling water used at K-Basins and shoreline seepage of groundwater that has passed near the retired 1301-N and 1325 cribs in the 100-N Area. Radionuclides released to the river from the 100-Area activities include tritium, ${ }^{90} \mathrm{Sr},{ }^{238} \mathrm{Pu},{ }^{235 / 240} \mathrm{Pu}$, and ${ }^{241} \mathrm{Am}$. These radionuclies are remnants of past disposal activities.

\subsection{Federal and State Radionuclide Air Emissions Standards}

U.S. Department of Energy facilities are also required to demonstrate compliance with the Clean Air Act National Emission Standards for Hazardous Air Pollutants (NESHAP) for radionuclides, as published in the 1989 amendments to 40 CFR Part 61, Subpart H, "National Emission Standards for Emissions of Radionuclides Other Than Radon From Department of Energy Facilities." The corresponding Washington State regulations appear in WAC 246-247, "Radiation Protection - Air Emissions," and WAC 173-480, “Ambient Air Quality Standards and Emission Limits for Radionuclides."

Radionuclide air emissions standards set by both the Federal EPA and Washington State regulations consist of calculated annual dose limits for an individual who could receive the maximum exposure to the emissions. As currently implemented, the Federal EPA standard in 40 CFR Part 61, Subpart H, is the most restrictive of the Federal and State standards with regard to radionuclide air emissions from DOE facilities. The standard, set forth in Section 61.92 of Subpart H, is as follows: "Emissions of radionuclides to the ambient air from Department of Energy facilities shall not exceed those amounts that would cause any member of the public to receive in any year an effective dose equivalent of $10 \mathrm{mrem} / \mathrm{yr}$." Washington State regulations either impose standards that are less restrictive or incorporate the Federal standard by reference.

The EPA regulations in 40 CFR Part 61, Subpart H, are designed to address routine emissions and do not specify standards for doses from short-term or high-level releases. However, WAC 246-247-040 (6) applies this standard to certain non-routine emissions as follows: "All emissions of radionuclides, including those due to emergency conditions resulting from startup, shutdown, maintenance activities, or process upsets are subject to the standards of this section and, therefore, subject to the enforcement actions of WAC 246-247-100."

\subsection{Methods for Demonstrating Compliance}

The December 15, 1989, revision to the Clean Air Act (40 CFR Part 61, Subpart H) requires DOE facilities to estimate the dose to a member of the public for radionuclides released from all potential sources of airborne radionuclides. The DOE and the EPA have interpreted the regulation to include diffuse and unmonitored sources as well as monitored point sources. The EPA has not specified or approved methods to estimate emissions from diffuse sources, and standardization is difficult because of the wide variety of such sources at DOE sites. Estimates of potential diffuse source emissions at Hanford were developed using environmental surveillance measurements of airborne radionuclides at the site perimeter. 
Regulatory requirements for determining compliance with the radionuclide air emissions standards likewise refer to methods specified in the Federal EPA standard. The methods specified in 40 CFR 61.92(a) include: “... EPA approved sampling procedures, computer models CAP-88 or AIRDOS-PC, or other procedures for which EPA has granted prior approval. DOE facilities for which the maximally exposed individual lives within 3 kilometers of all sources of emissions in the facility, may use EPA's COMPLY model and associated procedures for determining dose for purposes of compliance."

WAC 246-247 adopts by reference the approved methods specified in 40 CFR Part 61, Subparts H and I. WAC 173-480 refers to methods specified in WAC 402-80, which was subsequently recodified as WAC 246-247, or to "other methods that department of social and health services has determined to be suitable."

\subsection{Point of Compliance}

For U.S. Department of Energy facilities, the point of compliance has historically been a location at or near the site boundary that could be occupied for a substantial fraction of a year, and where an individual would receive the maximum combined dose from all facilities at the site. The point of compliance was based on 40 CFR Part 61, Subpart H, Section 61.94(a), which provides that "compliance with this standard shall be determined by calculating the highest effective dose equivalent to any member of the public at any offsite point where there is a residence, school, business or office." Washington State regulations are somewhat less specific in defining the point of compliance, but historically have been applied in a manner consistent with the Federal EPA regulation.

The regulatory agencies' definitions of what constitutes a DOE "facility" are also related to the point at which compliance is assessed. For purposes of the Federal EPA regulation, as defined in Section 61.91(b), "Facility means all buildings, structures and operations on one contiguous site." The Washington State definition in WAC 246-247-030 (11) is somewhat more restrictive: " 'Facility' means all buildings, structures, plants, processes, and operations on one contiguous site under control of the same owner or operator." Within each facility, there may be a number of "emission units," which are defined in Section 030(10) as "... any single location that emits or has the potential to emit airborne radioactive material. This may be a point source, nonpoint source, or source of fugitive emissions."

\subsection{Permitting New or Modified Sources of Radionuclide Emissions}

For purposes of permitting new (or modified) sources of radionuclide emissions, DOE sites are required by the EPA and by Washington State regulations to estimate doses to an MEI for the particular facility or "emission unit" under consideration. These dose estimates are performed to establish emission monitoring requirements for the facility, rather than to demonstrate compliance with annual dose standards. This evaluation also differs from the annual compliance calculations in that the MEI location is where the individual receives maximum dose from radionuclides released at the specific facility, rather than from all sources within the site boundary. The radionuclide release estimates, referred to as the "potential to emit" (PTE), are conservatively based on projected future emissions from the facility, assuming normal operations, but in the absence of any emission control devices. If the dose calculated for a PTE source at the facility is greater than $1 \%$ of the annual dose limit (or $0.1 \mathrm{mrem} / \mathrm{year}$ ), the facility 
is required to implement a continuous monitoring system that meets quality assurance standards specified in the 40 CFR Part 61 regulations. Emission monitoring requirements are less restrictive for facilities that fall below $0.1 \mathrm{mrem} / \mathrm{year}$ for the PTE. 


\subsection{Results of Recent Dose Assessments for Members of the Public From Hanford Site Operations}

Each year, the Pacific Northwest National Laboratory receives effluent release data from operating facilities within site boundaries. The data for both liquid effluents and atmospheric releases, are entered into the computer model GENII (Napier et al. 1988) to assess potential impacts to a hypothetical MEI. The MEI is a hypothetical person who resides at a single location and has a life style that maximizes exposure to the radionuclides released from Hanford facilities, e.g., historically, the MEI has been a resident farmer who is located near the Hanford Site boundary, but clearly offsite, and whose exposure pathways (see Figure 3.1) include direct exposure via water (swimming, boating, and fishing), soil

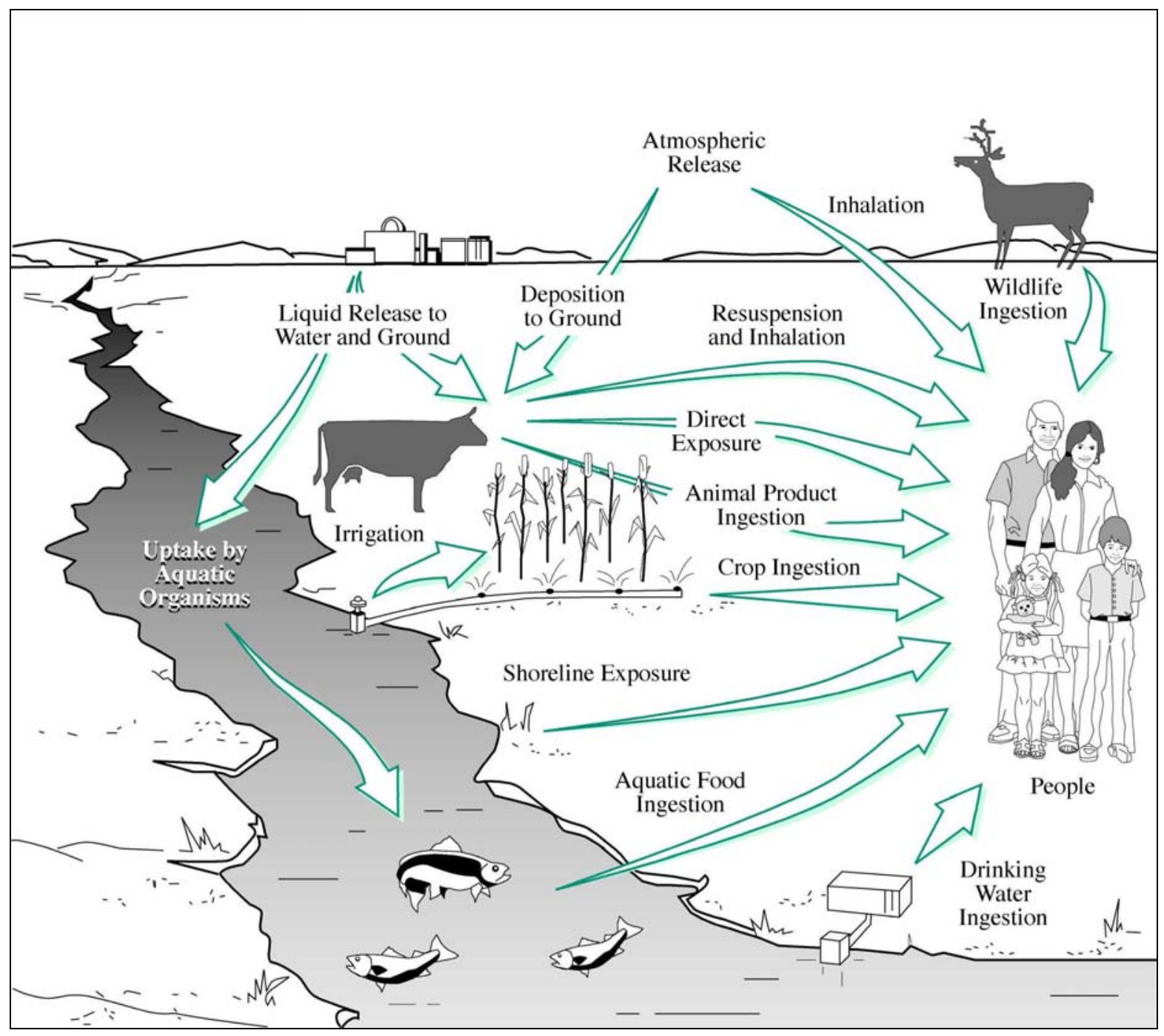

Figure 3.1. Potential Radiological Exposure Pathways to Humans 
(surface and buried sources), air submersion, inhalation, and ingestion. That location for recent annual release estimates has been across the Columbia River from the 300 Area in the Sagemoor area of Franklin County (see Figure 3.2).

Over the last 20 years, the Hanford mission has changed from nuclear material production to environmental research, waste management, and decommissioning and remediation of facilities and wastes from former production operations. As a result of the change in mission, site operations are being consolidated into fewer facilities, and areas of the site previously controlled by DOE are being released for other uses. This would allow members of the public access to Hanford Site areas that were previously restricted. Access to the Hanford Site for non-DOE-related employment necessitates review of pathway analysis for radiological dose to members of the public who may routinely occupy locations within the site boundary.

This section presents MEI doses calculated over the last 8 years, which have been reported in annual Hanford Site Environmental Reports (e.g., Poston et al. 2000). Parameters used in determining MEI doses are presented in Appendix A.

\subsection{Maximally Exposed Individual}

Historically, the MEI was situated either near the Ringold area, because of dose contributions from effluents released to the atmosphere from the 200 Area plateau, or in the Riverview area, because of dose contributions from the river pathway. In 1993, increases in atmospheric releases from 300 Area monitored stacks warranted the evaluation of a new MEI location, $1.5 \mathrm{~km}$ east of the 300 Area, across the Columbia River. The MEI has remained in this new location (at Sagemoor) for 7 years. A conservative assumption was made that the diet of the MEI residing across from the 300 Area consisted totally of foods purchased from the Riverview area, which could contain radionuclides present in both liquid and gaseous effluents from Hanford. The added contribution of the radionuclides in the Riverview irrigation water maximized the calculated dose from all air and water pathways. In 2000, the MEI location moved from the Sagemoor area to the Riverview area, indicating an increased contribution to dose from the water pathway, relative to the air pathway (Figure 3.3).

Radiological doses to the MEI ranged from a low of $0.007 \mathrm{mrem} / \mathrm{y}$ to a maximum of $0.01 \mathrm{mrem} / \mathrm{y}$ calculated in 1997 and 2000. Table 3.1 summarizes calculated doses to the hypothetical MEI from Hanford effluents. Figure 3.3 is graphical representation of the data. A graphical representation of dose to the MEI by specific pathway is shown in Appendix B. Atmospheric release data are tabulated in Appendix C.

The variation in the calculated MEI doses is minimal when compared to the variation in natural background radiation levels. Thermoluminescent dosimeter (TLD) surveillance data show variations around 5 mrem per year are possible at a given location. The TLD data coincides with the National Council on Radiation Protection and Measurements Report 94 (1987) that terrestrial radiation can vary 15 to $25 \%$ based solely on soil moisture. 


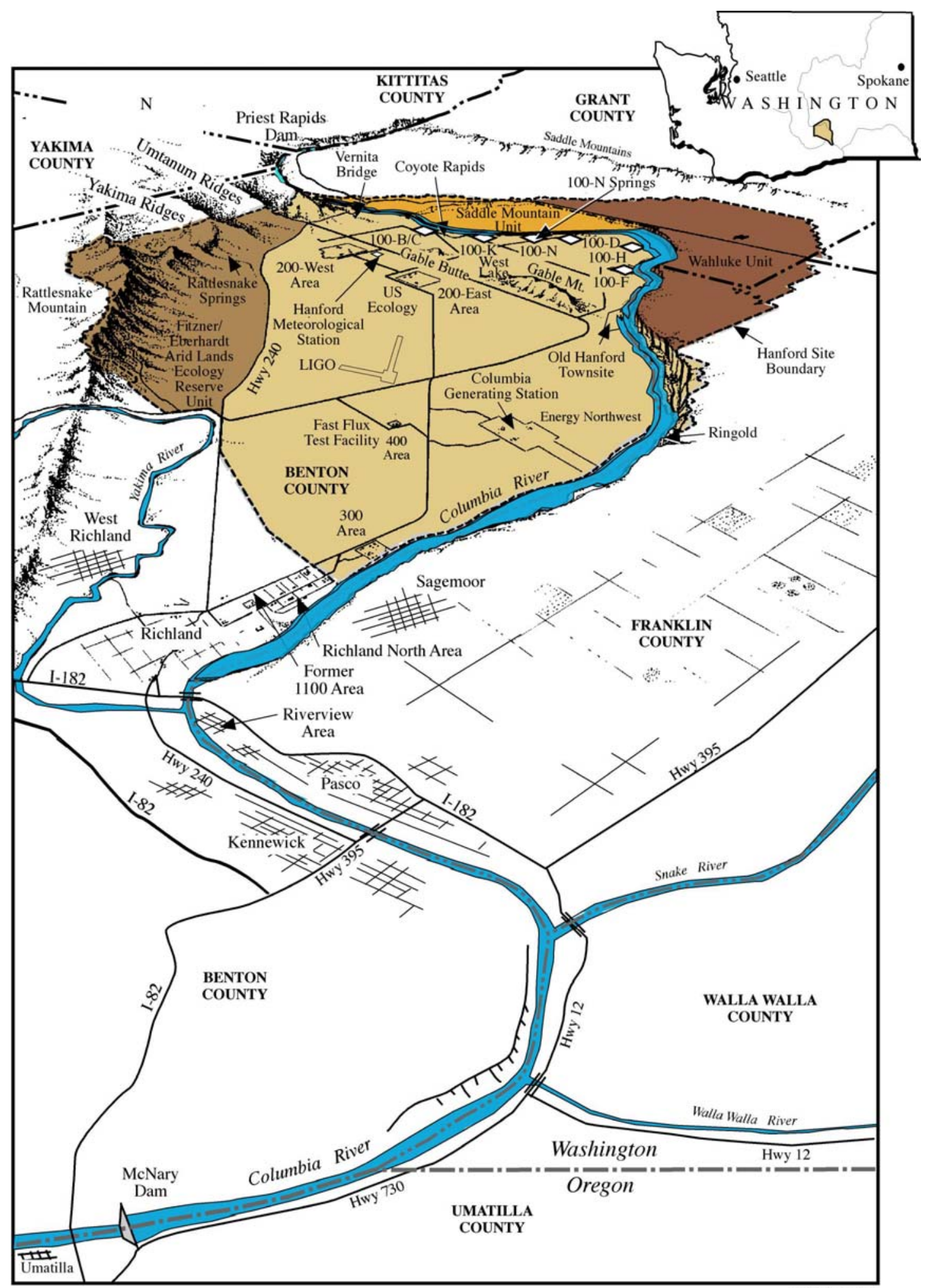

G02010082.1

Figure 3.2. Hanford Site and Surrounding Area 
Table 3.1. Potential Radiological Doses to Hypothetical Maximally Exposed Individual

\begin{tabular}{|c|c|c|c|c|c|c|}
\hline \multirow{2}{*}{$\begin{array}{c}\text { Year } \\
\text { (Location) }\end{array}$} & \multirow[b]{2}{*}{ Effluent } & \multicolumn{5}{|c|}{ Operating Area Contribution to Dose (mrem) } \\
\hline & & 100 Area & 200 Area & 300 Area & 400 Area & Total $^{(\mathbf{a})}$ \\
\hline \multirow{2}{*}{$\begin{array}{c}1993 \\
\text { (Sagemoor) }\end{array}$} & Air & $8 \mathrm{E}-06$ & $1 \mathrm{E}-03$ & $1 \mathrm{E}-02$ & $4 \mathrm{E}-05$ & \multirow{2}{*}{0.02} \\
\hline & Water & $8 \mathrm{E}-04$ & $1 \mathrm{E}-02$ & $2 \mathrm{E}-04$ & $--^{(b)}$ & \\
\hline \multirow{2}{*}{$\begin{array}{c}1994 \\
\text { (Sagemoor) }\end{array}$} & Air & $2 \mathrm{E}-05$ & $2 \mathrm{E}-03$ & $1 \mathrm{E}-02$ & $2 \mathrm{E}-05$ & \multirow[t]{2}{*}{0.05} \\
\hline & Water & 7 E-04 & 3 E-02 & $1 \mathrm{E}-05$ & --- & \\
\hline \multirow{2}{*}{$\begin{array}{c}1995 \\
\text { (Sagemoor) }\end{array}$} & Air & $1 \mathrm{E}-04$ & $1 \mathrm{E}-03$ & $5 \mathrm{E}-03$ & $1 \mathrm{E}-05$ & \multirow[t]{2}{*}{0.02} \\
\hline & Water & $1 \mathrm{E}-03$ & $2 \mathrm{E}-01$ & --- & --- & \\
\hline \multirow{2}{*}{$\begin{array}{c}1996 \\
\text { (Sagemoor) }\end{array}$} & Air & $1 \mathrm{E}-05$ & $8 \mathrm{E}-04$ & $4 \mathrm{E}-03$ & 4 E-05 & \multirow[t]{2}{*}{0.007} \\
\hline & Water & $6 \mathrm{E}-04$ & $2 \mathrm{E}-03$ & --- & --- & \\
\hline \multirow{2}{*}{$\begin{array}{c}1997 \\
\text { (Sagemoor) }\end{array}$} & Air & $3 \mathrm{E}-06$ & $1 \mathrm{E}-04$ & $3 \mathrm{E}-03$ & 8 E-05 & \multirow[t]{2}{*}{0.01} \\
\hline & Water & 6 E-04 & $7 \mathrm{E}-03$ & --- & --- & \\
\hline \multirow{2}{*}{$\begin{array}{c}1998 \\
\text { (Sagemoor) }\end{array}$} & Air & $7 \mathrm{E}-06$ & $2 \mathrm{E}-04$ & $1 \mathrm{E}-02$ & $1 \mathrm{E}-04$ & \multirow[t]{2}{*}{0.02} \\
\hline & Water & $2 \mathrm{E}-03$ & $6 \mathrm{E}-03$ & --- & --- & \\
\hline \multirow{2}{*}{$\begin{array}{c}1999 \\
\text { (Sagemoor) }\end{array}$} & Air & $2.5 \mathrm{E}-06$ & $3.0 \mathrm{E}-04$ & $5.5 \mathrm{E}-03$ & $1.5 \mathrm{E}-05$ & \multirow[t]{2}{*}{0.008} \\
\hline & Water & $3.1 \mathrm{E}-04$ & $1.8 \mathrm{E}-03$ & --- & --- & \\
\hline \multirow{2}{*}{$\begin{array}{c}2000 \\
\text { (Riverview) }\end{array}$} & Air & $2.2 \mathrm{E}-06$ & $7.3 \mathrm{E}-04$ & $1.4 \mathrm{E}-03$ & $6.4 \mathrm{E}-06$ & \multirow[t]{2}{*}{0.01} \\
\hline & Water & $1.5 \mathrm{E}-03$ & $1.0 \mathrm{E}-02$ & --- & --- & \\
\hline
\end{tabular}

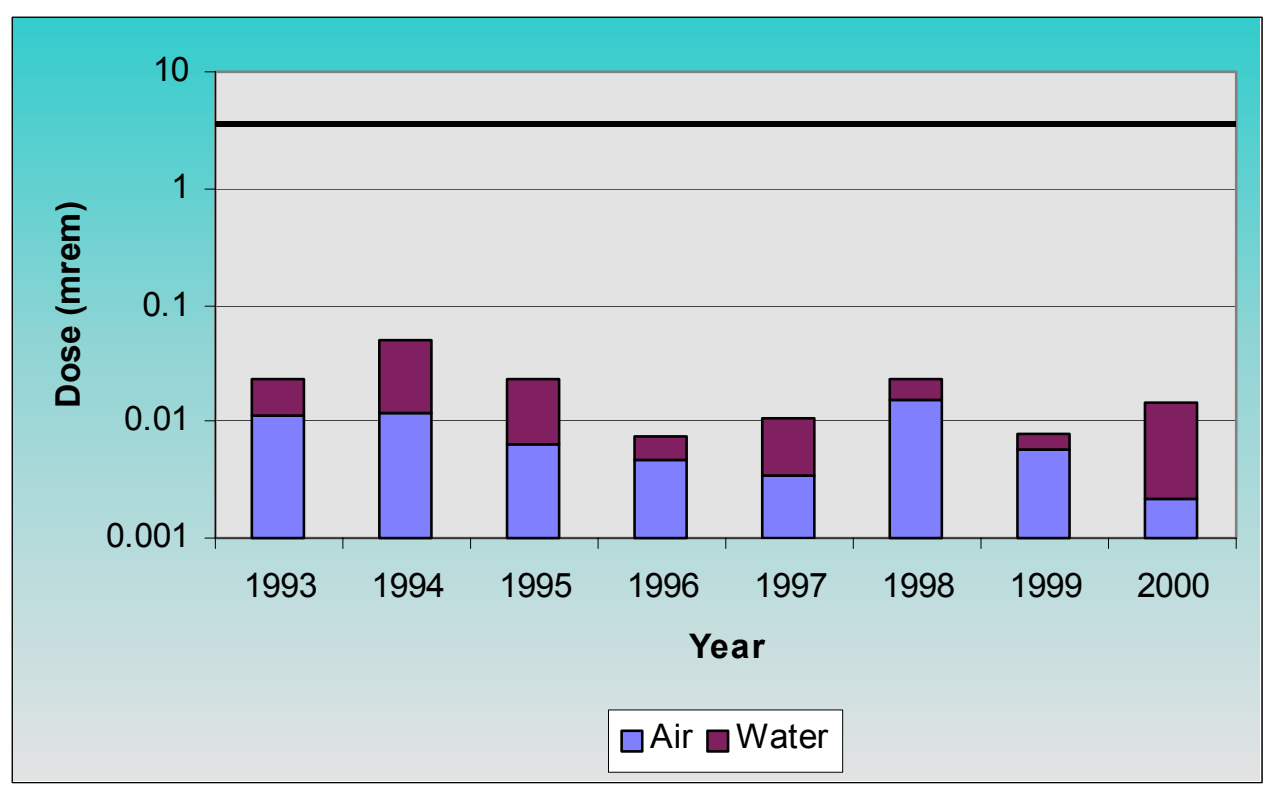

Figure 3.3. Calculated Radiological Doses to the MEI, $1.5 \mathrm{~km}$ East of the 300 Area from 1993 through 1999, and 2000 at Riverview - Line at 5 mrem is annual variation in natural background. 


\subsection{Current and Future Considerations}

Locations for evaluating doses to members of the public are currently being re-evaluated to determine whether the changing scope of DOE activities at Hanford and release of portions of the site or its facilities for other uses might require changes in assumptions or receptor locations. In addition, changes to the surrounding community may influence the location of individuals receiving maximum exposure to Hanford Site effluents. Ongoing revision of regulatory requirements and new interpretations of current regulations by agencies could also affect the methods used at the site for demonstrating compliance with those regulations.

\subsection{Changing Hanford Site Missions and Activities}

As noted previously, strategic nuclear materials production operations at Hanford, the site's major historic mission, have been shut down since 1987. Operation of the production reactors and chemical separation facilities accounted for the majority of radionuclide emissions to both water and air during the previous years. The site is now engaged in deactivating and cleaning up facilities and the environmental contamination resulting from previous activities. Continuing historic missions at the site include waste management as well as energy-related and environmental research. Fundamental changes in the type and scope of activities conducted at the site may result in public access to areas that have been restricted in the past. The patterns of radioactive material emissions from onsite facilities would also be expected to vary over time as the clean-up progresses and as the emphasis of ongoing waste management and research activities changes. For example, the shift in location of Hanford's maximally exposed offsite individual from the historic locations at Ringold or Riverview to a location across from the 300 Area was because of reduced emissions from the inactive production facilities in the 100 and 200 Areas and initiation of new research projects in the 300 Area.

\subsection{Environmental Restoration Activities}

Clean-up of inactive nuclear material production facilities and environmental restoration activities have been prioritized to focus on the Columbia River corridor, and to transfer most nuclear material management operations to the Hanford central plateau (the 200 Areas). Decontamination and decommissioning of the production reactors and fuel storage basins in the 100 Areas along the river is proceeding, and the remaining spent nuclear fuel in 100-K Area basins is being stabilized for storage in the 200 Areas. Some of the production reactor fuel fabrication facilities and research laboratories in the 300 Area are also being deactivated. Production reactor fuel components and some other nuclear materials stored in the 300 Area are being shipped offsite or removed to the 200 Areas for storage or disposal. Eventually, most of the nuclear material reprocessing facilities in the 200 Areas will be deactivated, as well, and the central plateau will be dedicated to long-term waste management and radioactive materials storage. In the interim, activities in the 200 Areas will continue to focus on stabilization and management of legacy wastes and other materials from the production facilities. A major effort that could significantly increase future atmospheric emissions is the retrieval and treatment of Hanford's high-level radioactive waste, stored in underground tanks. Currently, DOE plans to vitrify 
and store this waste in the 200 Areas until the repository for permanent disposal of high-level waste is completed. Emissions from treatment of the waste could increase the MEI dose from Hanford's air emissions by factors of 10 or more (DOE and WADOE 1996). As the environmental restoration and material stabilization operations proceed, emissions from the site could be expected to change, depending on the nature, duration, and location of ongoing activities.

\subsection{New and Ongoing Research Projects}

Ongoing research activities at Hanford are generally conducted in the 300 Area and the North Richland complex, with a few activities remaining in the 200 Areas or other parts of the site. Some new activities, such as production of medical and research isotopes, have also been proposed for the 400 Area, which houses facilities originally constructed to support research and development of nuclear breeder reactor technology. If the Fast Flux Test Facility is restarted to produce medical isotopes, both long-lived and short-lived radioactive effluents will be released to the atmosphere. These research facilities are located relatively nearer to offsite populations than the production facilities in the 100 and 200 Areas, which had historically been the major sources of public exposure to airborne radioactive effluents. The nature of ongoing research is also subject to change over the short term as new projects are initiated and ongoing projects are completed. Because of these factors, emissions from research facilities have the potential to influence the overall dose from Hanford Site activities to a greater degree than in the past.

\subsection{Leasing and Privatization of Hanford Site Facilities and Activities}

As Hanford facilities are deactivated and cleaned up, those that have no immediate use for DOE missions may be offered to private commercial operations or other government agencies for use in light industrial and research activities. Currently, a commercial metal products fabrication company occupies part of the 313 Building in the northern end of the 300 Area, and Washington State University leases laboratory space in buildings on the western side of the 300 Area. In addition, larger areas of the Hanford Site are currently occupied by non-DOE businesses or agencies under long-term leases or permits for purposes not directly related to DOE operations. A commercial low-level nuclear waste disposal facility is located immediately southwest of the 200-E Area, and a commercial nuclear power plant occupies an area along the eastern border of the Hanford Site on the Columbia River. The National Science Foundation constructed the Laser Interferometer Gravitational Wave Observatory (LIGO), a research facility in the southeastern part of the site near the 400 Area. Although these operations are located within the existing Hanford Site boundary, they are not staffed by DOE or its contractors' employees. The status of the staff at these facilities relative to radiation standards for members of the public is currently under discussion with the cognizant regulatory agencies.

\subsection{Release of Hanford Lands for Public Use}

In recent years, the shut-down of production operations at Hanford and the reduced level of activities at the site have resulted in greater public access to parts of the site managed by DOE and in some areas being released from DOE control. Former DOE facilities in the 1100 Area have been released to Benton County, which in turn has leased some of these facilities to private companies. The Fitzner/Eberhardt 
Arid Lands Ecology (ALE) Reserve, a 312 square kilometer (120 square mile) tract of land along the southwestern edge of the site, is managed jointly by the U.S. Fish and Wildlife Service and DOE. Two zones north of the Columbia River are also managed by other government agencies that have employees in the area on an occasional basis: 1) the Saddle Mountain Unit, a 130 square kilometer (50 square mile) tract of land located north-northwest of the Columbia River and generally south and east of State Highway 24, and 2) the Wahluke Unit, a 225 square kilometer (87 square mile) tract of land located north and east of both the Columbia River and the Saddle Mountain Unit (see Figure 3.2). These three units, along with sections of land still under DOE control, comprise the Hanford Reach National Monument. The portion of the monument administered only by DOE includes the McGee/Riverlands area (west of Vernita Bridge rest stop and north of State Highway 24) and the Columbia River islands of Benton County.

The creation of the Hanford Reach National Monument in 2000 may eventually result in greater public access to areas within the current site boundary as environmental restoration projects are completed. Although these changes do not necessarily affect site emissions or activities in themselves, they can result in members of the public traversing and working in areas of the site nearer to ongoing operations than in the past.

\subsection{Community Growth and Development}

Changes in the communities surrounding the Hanford Site also have the potential to affect levels of exposure to offsite individuals and populations. There has been substantial development of light industry, recreational facilities, and residential areas in the Horn Rapids area along the Yakima River near the southeast corner of the site. Portions of Franklin County adjacent to the eastern side of the site have also experienced residential development, although that area remains predominantly agricultural. Population distributions used to estimate collective dose from Hanford activities are typically updated at 10-year intervals with each general census. However, re-evaluation of doses to individuals in these developing areas may be warranted to ensure that they remain lower than those at the locations historically considered for the offsite MEI.

\subsection{Regulatory Environment}

The changing regulatory environment may also influence how the site demonstrates compliance with various radiation protection standards. Regular review and revision of regulations, and delegation of regulatory authority from Federal to State agencies may result in changes to the regulatory standards themselves, or in new interpretations and applications of existing standards. For example, DOE, the EPA, and the Nuclear Regulatory Commission (NRC) have all revised, or proposed revisions to, various regulations for radiation protection of the public over the last 10 years. In addition, state agencies having regulatory authority for public radiation protection have promulgated their own regulations in addition to those of the Federal agencies. As with the recent re-interpretation of the MEI location by State and Federal regulatory agencies, future changes to regulations that govern activities at DOE facilities would likewise require evaluation of the methods used to demonstrate compliance with the revised standards. 
The status of facilities subject to similar radiation protection standards, but which are regulated by different agencies, is also a question that remains to be resolved. For example, the status of employees at commercial facilities licensed by the NRC relative to emissions from DOE facilities has surfaced periodically as a concern. Employees at NRC-licensed facilities within the Hanford Site boundary have historically not been considered members of the public for demonstrating compliance with radiation protection standards because access to those facilities is restricted, and because their staff is subject to regulations regarding radiological protection and safety training equivalent to standards that govern DOE employees. 


\subsection{Potential Consequences for Exposure, Dose, and Risk Assessment}

DOE Order 5400.5 defines "public dose" as dose received by member(s) of the public from exposure to radiation and to the radioactive material released by a DOE facility or operation, whether the exposure is within a DOE site boundary or offsite. As a measure of this public dose, an MEI is a member of the public who has a lifestyle such that the dose from all pathways tends to be maximized. Historically, the MEI has been a resident farmer living near the down wind and downstream site boundary. However, because of increased access to areas that were formerly closed to the public (e.g., the Kaiser operation and the WSU laboratory in the 300 Area) and other non-DOE facilities within the site boundary (e.g., the Energy Northwest and LIGO facilities), the Washington State Department of Ecology, and the U.S. Environmental Protection Agency have requested DOE to consider the dose to an onsite maximally exposed member of the public in areas where DOE does not control access. These individuals are assumed to work full-time at one of the non-DOE facilities on the Hanford Site and to live in one of the surrounding communities.

\subsection{Evaluation of Dose to the Offsite Resident}

For 7 of the last 8 years, the offsite resident MEI has been located approximately $1.5 \mathrm{~km}$ east of the 300 Area, in the Sagemoor area of Franklin County. In other years, the MEI had been located at either Ringold or Riverview, depending on the nature and quantity of radionuclides in effluents released to the air and to the Columbia River from Hanford Site operations. The principal differences between those two MEI locations are the modes of exposure that determine the dose from Hanford effluents. The MEI at Ringold receives maximum exposure to emissions from Hanford 200 Area facilities that had been major contributors of airborne effluents in the past, but does not drink or consume foods irrigated with water from the Columbia River. The MEI at Riverview is farther from Hanford's major sources of airborne radionuclides but is exposed to radionuclides in liquid effluents by consumption of drinking water and irrigated foods derived from the Columbia River. The recent change in the MEI location to Sagemoor was due largely to a reduction in liquid and airborne effluents from the 100 and 200 Areas. The Sagemoor MEI receives maximum exposure to airborne effluents from 300 Area facilities, which have become relatively more important contributors to offsite dose than the decreasing 100 and 200 Area emissions.

The location of the resident MEI may be expected to change depending on the nature of ongoing activities at the site. The maximum dose to an offsite receptor will depend on the types and relative quantities of radionuclides in effluents from Hanford facilities. The location of maximum dose to an offsite resident member of the public is re-evaluated annually in determining compliance with DOE dose standards. The locations of maximum offsite dose from airborne effluents for operations at major Hanford areas are identified in Hill and Rittman (1999). 


\subsection{Methods for Evaluating Dose to an Onsite Maximally Exposed Individual}

Potential doses to receptors at onsite businesses were estimated for routine airborne effluents from all major operating areas at the Hanford Site. Releases of radionuclides to soil and surface water from Hanford facilities have largely been eliminated and are generally not subject to rapid change with ongoing activities at the site. Therefore, this evaluation focuses on atmospheric emissions from Hanford facilities, which are expected to exhibit the greatest variability and the highest potential to affect public receptors within the site boundary. The dose from atmospheric emissions was estimated using the CAP88-PC software (Parks 1992). The onsite doses were then compared to the dose at an offsite residence for each radionuclide from the same source and release quantity $(1 \mathrm{Ci} / \mathrm{y}$ of each radionuclide in the CAP88-PC radionuclide database). The analysis considered releases from representative facilities at all major Hanford Site operations and remediation projects, consistent with those used to determine the maximum dose to an offsite resident in Hill and Rittmann (1999). In evaluating the onsite doses, representative release points were chosen for the 100, 200, and 400 Areas at the same locations used for the offsite analysis. For the 300 Area, all major stacks were evaluated as sources because of their proximity to potential onsite receptors (Figure 5.1).

Onsite receptors were assumed to occupy the identified non-DOE work locations for 2000 hours per year and to be exposed to air emissions via the inhalation and external (plume submersion and ground surface) pathways. For the remainder of the year, these individuals were assumed to be exposed via all pathways (including ingestion as well as inhalation and external) at rates typical of an "average" member of the population within $80 \mathrm{~km}(50 \mathrm{mi})$ of the source facility (referred to as the "regional ingestion" dose). Sitespecific assumptions used in the dose estimates are listed in Appendix D. The potential onsite receptors include employees at LIGO and Energy Northwest for 100, 200, and 400 Area sources. Employees at the Kaiser Aluminum extrusion facility (North 313 Building) and a WSU laboratory in the 300 Area (3746 Building) were considered as receptors for 300 Area sources. Table 5.1 identifies the onsite receptor that receives the greatest dose for each of the release points and their location relative to the source facility.

The dose per unit release by radionuclide was calculated for each source at the maximally exposed onsite receptor. The dose for the onsite receptor was then compared to the dose from the same source at the maximally exposed offsite resident in Hill and Rittmann (1999). Radionuclides that resulted in a higher dose to the onsite receptor than to the offsite resident were identified by comparing the two sets of calculations. Dose calculations were performed for the complete set of radionuclides in the CAP88-PC library for a release from the $200 \mathrm{~W}$ Area. Although many of these radionuclides are of no importance for current Hanford operations, there is a potential for new projects to release radionuclides that have not historically been found at the site. For example, research and medical isotopes include many short-lived radionuclides that would not be present in significant quantities in products from the former production operations. A smaller subset of the CAP88-PC radionuclide library was evaluated for the other major operating areas on the Hanford Site. The smaller radionuclide set included all radionuclides reported in air emissions from Hanford Site facilities over the past 10 years, in addition to a selection of radionuclides identified in the $200-\mathrm{W}$ Area calculations that resulted in a higher dose onsite than offsite. 


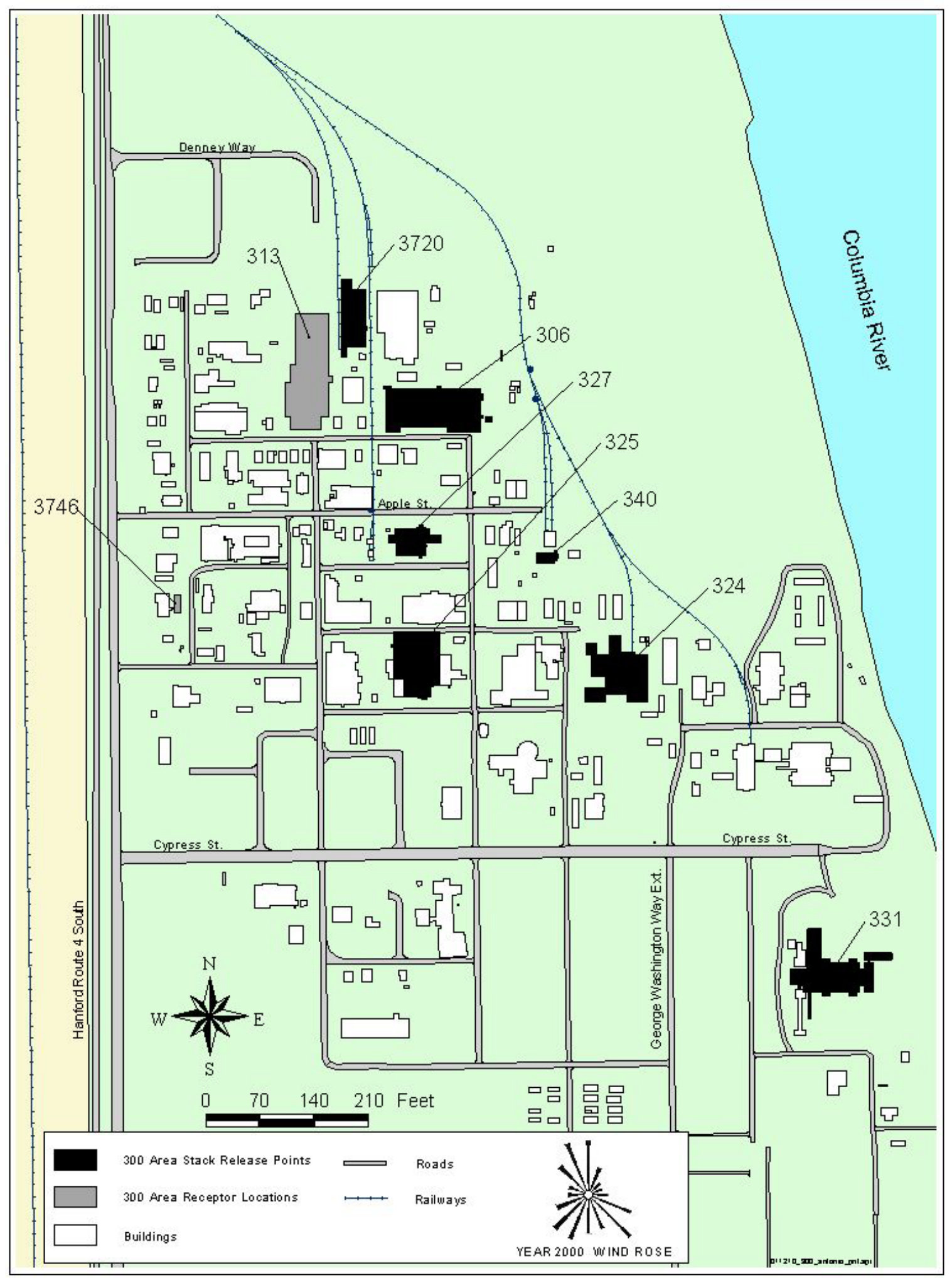

Figure 5.1. Stack Release Points and Onsite Receptors in 300 Area 
Table 5.1. Distances and Directions From Various Release Points to Potential Onsite Workers and Offsite Residents Being Evaluated for Potential MEI Status for Annual Compliance Reporting Purposes

\begin{tabular}{|c|c|c|}
\hline Release Point & $\begin{array}{c}\text { Onsite MEI Location } \\
\text { Distance and Direction }\end{array}$ & $\begin{array}{l}\text { Resident Offsite } \text { MEI }^{(\mathbf{a})} \\
\text { Distance and Direction }\end{array}$ \\
\hline $100-\mathrm{B} / \mathrm{C}$ & $\begin{array}{l}\text { LIGO } \\
26.3 \mathrm{~km} \mathrm{SE}\end{array}$ & $\begin{array}{l}\text { Site Boundary } \\
8.6 \mathrm{~km} \mathrm{NNW}\end{array}$ \\
\hline $100-\mathrm{D} / \mathrm{DR}$ & $\begin{array}{l}\text { LIGO } \\
27.9 \mathrm{~km} \mathrm{SSE}\end{array}$ & $\begin{array}{l}\text { Site Boundary } \\
8.9 \mathrm{~km} \mathrm{WNW}\end{array}$ \\
\hline $100-\mathrm{F}$ & $\begin{array}{l}\text { Energy NW } \\
22.4 \mathrm{~km} \mathrm{SSE}\end{array}$ & $\begin{array}{l}\text { Site Boundary } \\
9.7 \mathrm{~km} \text { ESE }\end{array}$ \\
\hline $100-\mathrm{H}$ & $\begin{array}{l}\text { Energy NW } \\
27.9 \mathrm{~km} \mathrm{SSE}\end{array}$ & $\begin{array}{l}\text { Site Boundary } \\
11.6 \mathrm{~km} \text { ESE }\end{array}$ \\
\hline $100-\mathrm{K}$ & $\begin{array}{l}\text { LIGO } \\
26.0 \mathrm{~km} \mathrm{SE}\end{array}$ & $\begin{array}{l}\text { Site Boundary } \\
8.9 \mathrm{~km} \mathrm{NNW}\end{array}$ \\
\hline $100-\mathrm{N}$ & $\begin{array}{l}\text { Energy NW } \\
28.8 \mathrm{~km} \mathrm{SE}\end{array}$ & $\begin{array}{l}\text { Site Boundary } \\
8.5 \mathrm{~km} \text { WNW }\end{array}$ \\
\hline $200-E$ & $\begin{array}{l}\text { Energy NW } \\
16.6 \mathrm{~km} \mathrm{ESE}\end{array}$ & $\begin{array}{l}\text { Site Boundary } \\
20.2 \mathrm{~km} \text { ESE }\end{array}$ \\
\hline $200-W$ & $\begin{array}{l}\text { LIGO } \\
18.6 \mathrm{~km} \text { ESE }\end{array}$ & $\begin{array}{l}\text { Site Boundary } \\
22 \mathrm{~km} \mathrm{SE}\end{array}$ \\
\hline 306 Building & $\begin{array}{l}313 \text { Building (Kaiser Aluminum Extrusion) } \\
140 \mathrm{~m} \mathrm{NW}\end{array}$ & $\begin{array}{l}\text { Site Boundary } \\
1.4 \mathrm{~km} \mathrm{NE}\end{array}$ \\
\hline 324 Building & $\begin{array}{l}313 \text { Building (Kaiser Aluminum Extrusion) } \\
540 \mathrm{~m} \mathrm{NW}\end{array}$ & $\begin{array}{l}\text { Site Boundary } \\
1.4 \mathrm{~km} \mathrm{NE}\end{array}$ \\
\hline 325 Building & $\begin{array}{l}3746 \text { Building (WSU Laboratory) } \\
290 \mathrm{~m} \text { WNW }\end{array}$ & $\begin{array}{l}\text { Site Boundary } \\
1.4 \mathrm{~km} \mathrm{NE}\end{array}$ \\
\hline 327 Building & $\begin{array}{l}313 \text { Building (Kaiser Aluminum Extrusion) } \\
250 \mathrm{~m} \mathrm{NNW}\end{array}$ & $\begin{array}{l}\text { Site Boundary } \\
1.4 \mathrm{~km} \mathrm{NE}\end{array}$ \\
\hline 331 Building & $\begin{array}{l}3746 \text { Building (WSU Laboratory) } \\
980 \mathrm{~m} \mathrm{NW}\end{array}$ & $\begin{array}{l}\text { Site Boundary } \\
1.1 \mathrm{~km} \mathrm{NE}\end{array}$ \\
\hline 340 Building & $\begin{array}{l}313 \text { Building (Kaiser Aluminum Extrusion) } \\
390 \mathrm{~m} \mathrm{NW}\end{array}$ & $\begin{array}{l}\text { Site Boundary } \\
1.4 \mathrm{~km} \mathrm{NE}\end{array}$ \\
\hline 3720 Building & $\begin{array}{l}313 \text { Building (Kaiser Aluminum Extrusion) } \\
70 \mathrm{~m} \mathrm{SSW}\end{array}$ & $\begin{array}{l}\text { Site Boundary } \\
1.4 \mathrm{~km} \mathrm{NE}\end{array}$ \\
\hline 400 Area & $\begin{array}{l}\text { Energy NW } \\
4.4 \mathrm{~km} \mathrm{NNE}\end{array}$ & $\begin{array}{l}\text { Site Boundary } \\
9.1 \mathrm{~km} \mathrm{SE}\end{array}$ \\
\hline
\end{tabular}

(a) For the purposes of these calculations, the distance and direction to the site boundary are used to represent a location that could be occupied by a full-time resident. The receptor location listed is the maximally exposed individual for releases from the specified release point (as opposed to the site MEI location for all Hanford emissions used for annual compliance calculations). 


\subsection{Results}

Potential doses from a simulated release at the 200-W Area for the complete CAP88-PC radionuclide library are listed in Appendix E for both the receptor at LIGO and the offsite MEI. A comparison of doses to the onsite and offsite receptors identified a small number of radionuclides that resulted in higher doses at the onsite location. Table 5.2 lists these radionuclides and their respective half-lives. The radionuclides with a higher dose at the onsite location consist of short-lived isotopes (half-lives less than 5 minutes), where the differences in dispersion and travel time to the onsite and offsite receptors offset the shorter exposure time at the onsite location.

The smaller radionuclide set was evaluated for reference facilities in the 100, 200, and 400 Areas, as identified in Hill and Rittmann (1999), and for major stacks in the 300 Area (including reference facilities for the east and west sectors of the 300 Area evaluated by Hill and Rittmann). Results of the simulations are presented in Appendix F. For the 100 Area sources and the east side of the 300 Area (331 Building), no radionuclides resulted in a greater dose to an onsite receptor than to the offsite resident. For the 200

Table 5.2. 200-West Area, Dose per Unit Release (mrem/y per $\mathrm{Ci} / \mathrm{y}$ ) for Onsite Worker at LIGO ${ }^{(a)}$ and Offsite Resident - CAP88-PC results by effective release height for radionuclides where onsite worker dose is greater than offsite resident dose.

\begin{tabular}{|c|c|c|c|c|c|}
\hline Release Height & & \multicolumn{2}{|c|}{$10 \mathrm{~m}$} & \multicolumn{2}{|c|}{$40 \mathrm{~m}$} \\
\hline Location & & 18.3 km ESE & $22 \mathrm{~km} \mathrm{SE}$ & $18.3 \mathrm{~km}$ ESE & $22 \mathrm{~km} \mathrm{SF}$ \\
\hline Nuclide & Half-life & $\begin{array}{c}\text { Onsite } \\
\text { Worker with } \\
\text { Regional } \\
\text { Ingestion }\end{array}$ & $\begin{array}{c}\text { Offsite } \\
\text { Resident }\end{array}$ & $\begin{array}{c}\text { Onsite } \\
\text { Worker with } \\
\text { Regional } \\
\text { Ingestion }\end{array}$ & $\begin{array}{c}\text { Offsite } \\
\text { Resident }\end{array}$ \\
\hline $\mathrm{O}-15$ & $122 \mathrm{~s}$ & $1.96 \mathrm{E}-14$ & $2.20 \mathrm{E}-15$ & $1.45 \mathrm{E}-14$ & $1.90 \mathrm{E}-15$ \\
\hline $\mathrm{Br}-85$ & $2.87 \mathrm{~m}$ & $1.98 \mathrm{E}-13$ & $6.00 \mathrm{E}-14$ & $1.47 \mathrm{E}-13$ & $5.30 \mathrm{E}-14$ \\
\hline Kr-89 & $3.15 \mathrm{~m}$ & $2.65 \mathrm{E}-11$ & $9.80 \mathrm{E}-12$ & $1.56 \mathrm{E}-11$ & $7.20 \mathrm{E}-12$ \\
\hline $\mathrm{Rb}-90$ & $2.6 \mathrm{~m}$ & $2.26 \mathrm{E}-12$ & $5.40 \mathrm{E}-13$ & $1.68 \mathrm{E}-12$ & $4.80 \mathrm{E}-13$ \\
\hline $\mathrm{Rb}-90 \mathrm{~m}$ & $4.3 \mathrm{~m}$ & $6.41 \mathrm{E}-10$ & $4.50 \mathrm{E}-10$ & $4.77 \mathrm{E}-10$ & $3.90 \mathrm{E}-10$ \\
\hline $\mathrm{Nb}-97 \mathrm{~m}$ & $52.7 \mathrm{~s}$ & $2.30 \mathrm{E}-22$ & $6.90 \mathrm{E}-25$ & $1.71 \mathrm{E}-22$ & $6.00 \mathrm{E}-25$ \\
\hline Rh-105m & $40 \mathrm{~s}$ & $6.98 \mathrm{E}-29$ & $7.70 \mathrm{E}-34$ & $5.18 \mathrm{E}-29$ & $6.80 \mathrm{E}-34$ \\
\hline Ag-109m & $39.6 \mathrm{~s}$ & $1.41 \mathrm{E}-33$ & $1.10 \mathrm{E}-37$ & $1.05 \mathrm{E}-33$ & $9.60 \mathrm{E}-38$ \\
\hline $\mathrm{Xe}-137$ & $3.82 \mathrm{~m}$ & $1.78 \mathrm{E}-11$ & $9.80 \mathrm{E}-12$ & $1.05 \mathrm{E}-11$ & $7.20 \mathrm{E}-12$ \\
\hline Ba-137m & $2.55 \mathrm{~m}$ & $3.81 \mathrm{E}-13$ & $8.60 \mathrm{E}-14$ & $2.83 \mathrm{E}-13$ & $7.50 \mathrm{E}-14$ \\
\hline Tl-207 & $4.77 \mathrm{~m}$ & $6.41 \mathrm{E}-12$ & $5.30 \mathrm{E}-12$ & $4.77 \mathrm{E}-12$ & $4.60 \mathrm{E}-12$ \\
\hline Tl-208 & $3.053 \mathrm{~m}$ & $2.37 \mathrm{E}-11$ & $8.50 \mathrm{E}-12$ & $1.77 \mathrm{E}-11$ & $7.40 \mathrm{E}-12$ \\
\hline T1-209 & $2.20 \mathrm{~m}$ & $1.51 \mathrm{E}-13$ & $2.20 \mathrm{E}-14$ & $1.13 \mathrm{E}-13$ & $1.90 \mathrm{E}-14$ \\
\hline Bi-211 & $2.14 \mathrm{~m}$ & $7.76 \mathrm{E}-14$ & $1.00 \mathrm{E}-14$ & $5.77 \mathrm{E}-14$ & $8.80 \mathrm{E}-15$ \\
\hline Fr-221 & $4.9 \mathrm{~m}$ & $3.08 \mathrm{E}-09$ & $2.60 \mathrm{E}-09$ & $2.28 \mathrm{E}-09$ & $2.30 \mathrm{E}-09$ \\
\hline $\mathrm{Pa}-234 \mathrm{~m}$ & $1.17 \mathrm{~m}$ & $9.26 \mathrm{E}-22$ & $7.80 \mathrm{E}-24$ & $6.89 \mathrm{E}-22$ & $6.80 \mathrm{E}-24$ \\
\hline $\begin{array}{l}\text { (a) Onsite dose a } \\
\text { pathways; re } \\
\text { population. }\end{array}$ & umes expo & $000 \mathrm{~h} / \mathrm{y}$ at the & $\mathrm{k}$ location & inhalation and e & $\begin{array}{l}\text { of the } \\
\text { ol }\end{array}$ \\
\hline
\end{tabular}


and 400 Areas and for elevated releases on the west side of the 300 Area (324 Building), only very shortlived nuclides resulted in greater onsite doses (Table 5.3). These radionuclides are not released in significant quantities by current operations at the Hanford Site, and, because they are short-lived, they produce a very low dose per unit release. Therefore, they would add little to the total dose for either an onsite or offsite receptor, compared to the radionuclides that are typically major contributors to the dose from air emissions at Hanford.

The results of simulations for the remaining 300 Area major stacks are also shown in Table 5.3. For 10 -m releases from the west side of the 300 Area, the dose to an onsite receptor was greater than to an offsite resident for a number of radionuclides routinely reported in emissions from Hanford Site operations. Therefore, the relative magnitude of doses to onsite and offsite receptors from these sources would depend on the types and quantities of radionuclides in the facility effluents. 
Table 5.3. Simulation Results of Radionuclides in CAP88 Library and Locations for which the Dose Rates per Unit Release (mrem/y per Ci/y) to Onsite Worker ${ }^{(a)}$ Exceeds Dose to Offsite Resident - All releases are at $10 \mathrm{~m}$ except as noted in table.

\begin{tabular}{|c|c|c|c|c|c|c|c|c|}
\hline \multirow[b]{4}{*}{ Nuclide } & \multicolumn{8}{|c|}{ Release Location } \\
\hline & \multicolumn{2}{|c|}{ 200-E Area } & \multicolumn{2}{|c|}{ 200-W Area } & \multicolumn{2}{|c|}{324 Bldg, 40-m Release } & \multicolumn{2}{|c|}{400 Area } \\
\hline & \multicolumn{8}{|c|}{ Receptor Location } \\
\hline & $\begin{array}{c}\text { Offsite } \\
\text { Resident }^{(\mathbf{b})} \\
(20.2 \text { km } \\
\text { ESE) }\end{array}$ & $\begin{array}{c}\text { Onsite } \\
\text { Worker, } \\
\text { Energy NW } \\
(16.6 \mathrm{~km} \\
\text { ESE) }\end{array}$ & $\begin{array}{c}\text { Offsite } \\
\text { Resident }^{(\mathbf{b})} \\
(22.0 \mathrm{~km} \\
\text { SE) }\end{array}$ & $\begin{array}{c}\text { Onsite } \\
\text { Worker } \\
\text { LIGO } \\
\text { (18.6 km } \\
\text { ESE) }\end{array}$ & $\begin{array}{c}\text { Offsite } \\
\text { Resident }^{(\mathbf{b})} \\
(1400 \mathrm{~m} \\
\text { NE) }\end{array}$ & $\begin{array}{c}\text { Onsite } \\
\text { Worker, } \\
\text { Energy } \\
\text { NW } \\
(540 \text { m } \\
\text { NW) }\end{array}$ & $\begin{array}{c}\text { Offsite } \\
\text { Resident }^{(\mathbf{b})} \\
(\mathbf{9 . 1} \text { km SE) }\end{array}$ & $\begin{array}{c}\text { Onsite } \\
\text { Worker, } \\
\text { Energy } \\
\text { NW } \\
(4.6 \text { km } \\
\text { NNE) }\end{array}$ \\
\hline $\mathrm{O}-15$ & $1.90 \mathrm{E}-14$ & $1.67 \mathrm{E}-13$ & $2.2 \mathrm{E}-15$ & $1.45 \mathrm{E}-14$ & & & $1.0 \mathrm{E}-09$ & 8.49E-08 \\
\hline Ag-110 & & & & & $3.90 \mathrm{E}-09$ & $1.17 \mathrm{E}-08$ & $4.8 \mathrm{E}-26$ & $1.60 \mathrm{E}-16$ \\
\hline T1-208 & $4.2 \mathrm{E}-11$ & $1.23 \mathrm{E}-10$ & $8.5 \mathrm{E}-12$ & $1.94 \mathrm{E}-11$ & & & $7.4 \mathrm{E}-08$ & $1.45 \mathrm{E}-06$ \\
\hline
\end{tabular}

\begin{tabular}{|c|c|c|c|c|c|c|c|}
\hline \multirow[b]{4}{*}{ Nuclide } & \multicolumn{7}{|c|}{ Release Location } \\
\hline & $\begin{array}{l}\text { 300-W Area } \\
\text { (HNF-3602) }\end{array}$ & 306 Bldg & 324 Bldg & 325 Bldg & 327 Bldg & 340 Bldg & 3720 Bldg \\
\hline & \multicolumn{7}{|c|}{ Receptor Location } \\
\hline & $\begin{array}{c}\text { Offsite } \\
\text { Resident }^{(\mathbf{b})} \\
\left(1400 \mathrm{~m} \mathrm{NE}^{2}\right)\end{array}$ & $\begin{array}{c}\text { Onsite } \\
313 \text { Bldg } \\
(140 \mathrm{~m} \mathrm{NW}) \\
\end{array}$ & $\begin{array}{c}\text { Onsite } \\
313 \text { Bldg } \\
(540 \mathrm{~m} \mathrm{NW}) \\
\end{array}$ & $\begin{array}{c}\text { Onsite } 3746 \\
\text { Bldg } \\
(290 \text { WNW }) \\
\end{array}$ & $\begin{array}{c}\text { Onsite } \\
313 \text { Bldg } \\
(250 \mathrm{~m} \text { NNW }) \\
\end{array}$ & $\begin{array}{c}\text { Onsite } \\
313 \text { Bldg } \\
(385 \text { m NW) } \\
\end{array}$ & $\begin{array}{c}\text { Onsite } \\
313 \text { Bldg } \\
\text { (70 m } \\
\text { SSW) } \\
\end{array}$ \\
\hline $\mathrm{O}-15$ & $4.30 \mathrm{E}-05$ & $4.31 \mathrm{E}-04$ & 7.71E-05 & $1.54 \mathrm{E}-04$ & $1.91 \mathrm{E}-04$ & $1.47 \mathrm{E}-04$ & $3.54 \mathrm{E}-04$ \\
\hline Ar-41 & $2.80 \mathrm{E}-04$ & $7.82 \mathrm{E}-04$ & $3.42 \mathrm{E}-04$ & $4.10 \mathrm{E}-04$ & $4.95 \mathrm{E}-04$ & $4.72 \mathrm{E}-04$ & $5.15 \mathrm{E}-04$ \\
\hline Mn-54 & $2.70 \mathrm{E}-01$ & 7.23E-01 & $3.12 \mathrm{E}-01$ & $3.73 \mathrm{E}-01$ & $4.51 \mathrm{E}-01$ & 4.31E-01 & 4.78E-01 \\
\hline Co-60 & $4.10 \mathrm{E}+00$ & $1.08 \mathrm{E}+01$ & $4.68 \mathrm{E}+00$ & $5.57 \mathrm{E}+00$ & $6.76 \mathrm{E}+00$ & $6.44 \mathrm{E}+00$ & $7.17 \mathrm{E}+00$ \\
\hline Zn-69m & $7.60 \mathrm{E}-04$ & $1.99 \mathrm{E}-03$ & $8.71 \mathrm{E}-04$ & $1.04 \mathrm{E}-03$ & $1.26 \mathrm{E}-03$ & $1.20 \mathrm{E}-03$ & $1.31 \mathrm{E}-03$ \\
\hline Kr-85 & $7.70 \mathrm{E}-07$ & $2.01 \mathrm{E}-06$ & $9.00 \mathrm{E}-07$ & $1.07 \mathrm{E}-06$ & $1.29 \mathrm{E}-06$ & $1.23 \mathrm{E}-06$ & $1.32 \mathrm{E}-06$ \\
\hline Zr-95 & $6.50 \mathrm{E}-02$ & $1.47 \mathrm{E}-01$ & & $7.64 \mathrm{E}-02$ & $9.23 \mathrm{E}-02$ & $8.80 \mathrm{E}-02$ & $9.78 \mathrm{E}-02$ \\
\hline $\mathrm{Nb}-95$ & $8.00 \mathrm{E}-02$ & $8.23 \mathrm{E}-02$ & & & & & \\
\hline $\mathrm{Ru}-103$ & $3.10 \mathrm{E}-02$ & $6.38 \mathrm{E}-02$ & & $3.30 \mathrm{E}-02$ & $4.01 \mathrm{E}-02$ & $3.83 \mathrm{E}-02$ & $4.24 \mathrm{E}-02$ \\
\hline Ru-106 & $2.70 \mathrm{E}-01$ & $4.84 \mathrm{E}-01$ & & & $3.08 \mathrm{E}-01$ & $2.94 \mathrm{E}-01$ & $3.19 \mathrm{E}-01$ \\
\hline $\mathrm{Ag}-110$ & $8.50 \mathrm{E}-09$ & $4.68 \mathrm{E}-06$ & $9.56 \mathrm{E}-08$ & $6.32 \mathrm{E}-07$ & $9.88 \mathrm{E}-07$ & $3.67 \mathrm{E}-07$ & $7.60 \mathrm{E}-06$ \\
\hline Sb-125 & 4.40E-01 & $1.16 \mathrm{E}+00$ & $5.02 \mathrm{E}-01$ & $6.00 \mathrm{E}-01$ & $7.28 \mathrm{E}-01$ & $6.92 \mathrm{E}-01$ & 7.71E-01 \\
\hline Cs-134 & $1.70 \mathrm{E}+00$ & $3.16 \mathrm{E}+00$ & & & $1.98 \mathrm{E}+00$ & $1.89 \mathrm{E}+00$ & $2.11 \mathrm{E}+00$ \\
\hline Cs-137+D & $3.97 \mathrm{E}+00$ & $9.68 \mathrm{E}+00$ & $4.19 \mathrm{E}+00$ & $5.01 \mathrm{E}+00$ & $6.07 \mathrm{E}+00$ & $5.77 \mathrm{E}+00$ & $6.43 \mathrm{E}+00$ \\
\hline $\mathrm{Ce}-144$ & $2.10 \mathrm{E}-01$ & $3.96 \mathrm{E}-01$ & & & $2.53 \mathrm{E}-01$ & $2.41 \mathrm{E}-01$ & $2.62 \mathrm{E}-01$ \\
\hline Pm-147 & $1.80 \mathrm{E}-02$ & $3.96 \mathrm{E}-02$ & & $2.08 \mathrm{E}-02$ & $2.53 \mathrm{E}-02$ & $2.41 \mathrm{E}-02$ & $2.60 \mathrm{E}-02$ \\
\hline Eu-152 & $4.00 \mathrm{E}+00$ & $1.10 \mathrm{E}+01$ & $4.75 \mathrm{E}+00$ & $5.66 \mathrm{E}+00$ & $6.87 \mathrm{E}+00$ & $6.53 \mathrm{E}+00$ & $7.28 \mathrm{E}+00$ \\
\hline Eu-154 & $3.30 \mathrm{E}+00$ & $8.85 \mathrm{E}+00$ & $3.83 \mathrm{E}+00$ & $4.58 \mathrm{E}+00$ & $5.54 \mathrm{E}+00$ & $5.27 \mathrm{E}+00$ & $5.88 \mathrm{E}+00$ \\
\hline Eu-155 & $1.30 \mathrm{E}-01$ & $3.48 \mathrm{E}-01$ & $1.51 \mathrm{E}-01$ & $1.80 \mathrm{E}-01$ & $2.18 \mathrm{E}-01$ & $2.08 \mathrm{E}-01$ & $2.31 \mathrm{E}-01$ \\
\hline T1-208 & $2.60 \mathrm{E}-04$ & $1.86 \mathrm{E}-03$ & $4.31 \mathrm{E}-04$ & $7.46 \mathrm{E}-04$ & $9.15 \mathrm{E}-04$ & $7.53 \mathrm{E}-04$ & $1.42 \mathrm{E}-03$ \\
\hline $\mathrm{Pb}-212$ & $6.40 \mathrm{E}-02$ & $1.73 \mathrm{E}-01$ & $7.63 \mathrm{E}-02$ & $9.09 \mathrm{E}-02$ & $1.10 \mathrm{E}-01$ & $1.05 \mathrm{E}-01$ & $1.14 \mathrm{E}-01$ \\
\hline Bi-212 & $1.20 \mathrm{E}-02$ & $3.45 \mathrm{E}-02$ & $1.46 \mathrm{E}-02$ & $1.79 \mathrm{E}-02$ & $2.16 \mathrm{E}-02$ & $2.05 \mathrm{E}-02$ & $2.29 \mathrm{E}-02$ \\
\hline U-234 & $5.30 \mathrm{E}+01$ & $1.34 \mathrm{E}+02$ & $5.92 \mathrm{E}+01$ & $7.06 \mathrm{E}+01$ & $8.52 \mathrm{E}+01$ & $8.13 \mathrm{E}+01$ & $8.82 \mathrm{E}+01$ \\
\hline U-235 & $5.10 \mathrm{E}+01$ & $1.28 \mathrm{E}+02$ & $5.64 \mathrm{E}+01$ & $6.72 \mathrm{E}+01$ & $8.13 \mathrm{E}+01$ & $7.74 \mathrm{E}+01$ & $8.40 \mathrm{E}+01$ \\
\hline U-238 & $4.70 \mathrm{E}+01$ & $1.19 \mathrm{E}+02$ & $5.28 \mathrm{E}+01$ & $6.28 \mathrm{E}+01$ & $7.58 \mathrm{E}+01$ & $7.24 \mathrm{E}+01$ & $7.85 \mathrm{E}+01$ \\
\hline $\mathrm{Pu}-239$ & $1.40 \mathrm{E}+02$ & $3.46 \mathrm{E}+02$ & $1.53 \mathrm{E}+02$ & $1.82 \mathrm{E}+02$ & $2.20 \mathrm{E}+02$ & $2.10 \mathrm{E}+02$ & $2.28 \mathrm{E}+02$ \\
\hline $\mathrm{Pu}-241$ & $2.20 \mathrm{E}+00$ & $5.27 \mathrm{E}+00$ & $2.33 \mathrm{E}+00$ & $2.78 \mathrm{E}+00$ & $3.35 \mathrm{E}+00$ & $3.19 \mathrm{E}+00$ & $3.47 \mathrm{E}+00$ \\
\hline Am-241 & $2.20 \mathrm{E}+02$ & $5.50 \mathrm{E}+02$ & $2.44 \mathrm{E}+02$ & $2.90 \mathrm{E}+02$ & $3.51 \mathrm{E}+02$ & $3.35 \mathrm{E}+02$ & $3.63 \mathrm{E}+02$ \\
\hline $\begin{array}{l}\text { (a) Onsit } \\
\text { repres } \\
\text { (b) The o } \\
\text { locati }\end{array}$ & $\begin{array}{l}\text { resident locat } \\
\text { or all Hanford }\end{array}$ & $\begin{array}{l}\text { listed is the } m \\
\text { ssions used for }\end{array}$ & $\begin{array}{l}\text { work location } \mathrm{fo} \\
\text { pulation by all } \mathrm{p} \\
\text { nally exposed in } \\
\text { qual compliance }\end{array}$ & $\begin{array}{l}\text { nhalation and ex } \\
\text { ways. } \\
\text { idual for release } \\
\text { culations). }\end{array}$ & $\begin{array}{l}\text { rnal pathways; re } \\
\text { from each release }\end{array}$ & $\begin{array}{l}\text { nder of year exp } \\
\text { int (as opposed t }\end{array}$ & he is \\
\hline
\end{tabular}




\subsection{Proposed Changes to Methods for Evaluating Exposure, Dose, and Risk}

The following sections describe proposed changes to the methods for assessing public dose and risk in permitting new facilities and in demonstrating compliance with requirements of public radiationprotection standards. Possible needs for dose assessments at additional receptor locations and for expansion of routine radiological monitoring are discussed.

\subsection{Need to Evaluate New Receptors}

Based on the analysis of potential radiological doses at onsite non-DOE operations, workers at some of these locations have the potential to receive a higher dose than the offsite resident typically evaluated in permitting new facilities or in demonstrating compliance with public dose standards. For most of the major operating areas on the Hanford Site, the dose to an offsite resident would be greater than the dose to any of the onsite public receptors identified in the analysis. However, for many sources within the 300 Area, onsite non-DOE receptors located in or near the 300 Area have the potential to receive higher doses than the offsite resident, depending on the types and quantities of radionuclides in the facility's airborne effluents. Therefore, these receptors should be evaluated on a case-by-case basis when permitting new operations in the 300 Area and in demonstrating compliance with public radiationprotection standards.

For sources of radionuclide emissions other than those in the 300 Area, the onsite public receptors identified in this analysis would be very unlikely to receive a greater dose than the offsite resident typically evaluated for those sources. At most onsite receptor locations, the lower exposure time reduces the dose to a level that more than offsets a potential increase from the receptor's proximity to potential emission sources. The exception would be for operations that could release substantial quantities of short-lived isotopes (those with half-lives less than 5 minutes) not present in recent effluents from Hanford facilities. Such operations would also need to be evaluated on a case-by-case basis to determine whether the dose for an onsite receptor could be greater than for an offsite resident.

Changes to the Hanford Site boundaries and leasing of land or facilities within the site to non-DOE operations could result in the need to establish new receptors for some permitting or compliance purposes. Changes in access to various parts of the site by non-DOE personnel should be evaluated where those individuals have the potential to spend substantial amounts of time within the historic Hanford Site boundary.

\subsection{Need for Additional Radiological Monitoring - Air or External}

The following sections address the possible need for additional environmental or facility monitoring capabilities based on the evaluation of regulatory and reindustrialization activities presented previously. 
These needs should be reassessed whenever new activities on the site could result in substantial changes to the types and quantities of airborne effluents released from Hanford facilities or in alternative land uses that change exposure pathways.

\subsubsection{Monitored Locations}

Based on the non-DOE receptors within the Hanford site boundary evaluated for this study, the existing environmental monitoring network should be adequate to provide assurance that regulatory standards are met for routine activities. There are air and/or external radiation monitoring stations near public receptors at the 300 Area, LIGO, and Energy Northwest facilities. Emissions from facilities that could release substantial quantities of radionuclides are also monitored at the source by one or more effluent sampling systems. Given the current types and quantities of radionuclides emitted from Hanford facilities, the combination of effluent and environmental monitoring is sufficient to characterize past or potential doses to public receptors within the site boundary. The need for special sampling should be considered where an operation could release radionuclides from a source or area that is not routinely monitored or that could release substantial quantities of radionuclides over a very short period of time. Additional monitoring should also be considered for future public receptors within the Hanford Site who are not in proximity to a location covered by the existing environmental monitoring network.

\subsubsection{Monitored Radionuclides}

The existing environmental and facility effluent monitoring capabilities are sufficient to characterize radionuclide emissions from ongoing Hanford operations. New activities that could release radionuclides not routinely monitored in either facility or environmental sampling programs would require evaluation for their possible impacts on dose to members of the public at both onsite and offsite locations. If the potential dose is sufficiently high, sampling of facility effluents for the major contributors to dose would be required, and the need for environmental monitoring of the isotopes should be assessed. Operations that could release substantial quantities of short-lived isotopes would require special consideration because the existing monitoring programs may not detect those radionuclides. Special sampling procedures designed to detect short-lived radionuclides may be needed commensurate with their potential dose to members of the public.

\subsubsection{Administrative Considerations}

In order to ensure that environmental monitoring locations and dose assessments for permitting new facilities and for demonstrating compliance with public dose standards are appropriate, a mechanism should be established to inform the organizations responsible for these activities as new operations are initiated on the Hanford Site. Communication between organizations has become more critical as the number of contractors performing work at Hanford has increased and as the DOE responsibilities for these contractors are divided between two independent organizations. Accurate monitoring of the effect of DOE operations on members of the public and on the environment depends on sufficient lead-time to implement procedures for detecting and measuring levels of radiation and radioactive material emissions from Hanford operations. 


\subsection{References}

40 CFR 61. U.S. Environmental Protection Agency. "National Emission Standards for Hazardous Air Pollutants."

40 CFR 141. U.S. Environmental Protection Agency. "National Primary Drinking Water Regulations."

Clean Air Act. 42 USC 7401 et seq.

Fluor Hanford (FH). 2001. Hanford Site Solid Waste Acceptance Criteria. HNF-EP-0063, Rev. 6, Fluor Hanford, Richland Washington.

Hill, J. S., and P. D. Rittmann. 1999. Volume 1: Calculating Potential to Emit Releases and Doses for FEMPs and NOCs. HNF-3602, Vol. 1, Fluor Daniel Hanford, Inc., Richland, Washington.

Napier, B. A., R. A. Peloquin, D. L. Strenge, and J. V. Ramsdell. 1988. GENII - The Hanford Environmental Radiation Dosimetry Software System, Vols. 1-3. PNL-6584, Pacific Northwest Laboratory, Richland, Washington.

National Council on Radiation Protection and Measurements (NCRP). 1987. Exposure of the Population in the United States and Canada from Natural Background Radiation. Report No. 94, Bethesda, Maryland.

Parks, B. S. 1992. User's Guide for CAP88-PC. Version 1.0. 402-B-92-001, Office of Radiation Programs, U.S. Environmental Protection Agency, Las Vegas, Nevada.

Poston, T.M., R.W. Hanf, and R.L. Dirkes, eds. 2000. Hanford Site Environmental Report, Calendar Year 1999. PNNL-13230, Pacific Northwest National Laboratory, Richland, Washington.

U.S. Department of Energy (DOE). 1990. "Radiation Protection of the Public and the Environment." DOE Order 5400.5 (February 2, 1990).

U.S. Department of Energy (DOE). 1996. Tank Waste Remediation System, Hanford Site. Environmental Impact Statement. DOE/EIS-0189, U.S. Department of Energy, Richland, Washington.

U.S. Department of Energy (DOE). 1999. “Radioactive Waste Management.” DOE Order 435.1 (July 9, 1999). 


\section{Appendix A}

\section{Parameters Used in Dose Calculations}




\section{Appendix A}

\section{Parameters Used in Dose Calculations}

Table A.1. Food Pathway Parameters Used in Dose Calculations

\begin{tabular}{|c|c|c|c|c|c|}
\hline \multirow[b]{2}{*}{ Medium } & \multicolumn{2}{|c|}{ Holdup, $\mathrm{d}^{(\mathrm{a})}$} & \multirow[b]{2}{*}{$\begin{array}{l}\text { Growing } \\
\text { Period, d }\end{array}$} & \multirow[b]{2}{*}{$\begin{array}{l}\text { Yield, } \\
\mathrm{kg} / \mathrm{m}^{2}\end{array}$} & \multirow[b]{2}{*}{$\begin{array}{c}\text { Irrigation Rate, } \\
\mathbf{L} / \mathbf{m}^{2} / \mathbf{m o}\end{array}$} \\
\hline & $\begin{array}{l}\text { Maximally Exposed } \\
\text { Individual }\end{array}$ & $\begin{array}{c}\text { Average } \\
\text { Individual }\end{array}$ & & & \\
\hline Leafy vegetables & 1 & 14 & 90 & 1.5 & 150 \\
\hline Other vegetables & 5 & 14 & 90 & 4 & 170 \\
\hline Fruit & 5 & 14 & 90 & 2 & 150 \\
\hline Cereal & 180 & 180 & 90 & 0.8 & 0 \\
\hline Eggs & 1 & 18 & 90 & 0.8 & 0 \\
\hline Milk & 1 & 4 & -- & -- & -- \\
\hline Hay & $(100)^{(b)}$ & $(100)$ & 45 & 2 & 200 \\
\hline Pasture & $(0)$ & $(0)$ & 30 & 1.5 & 200 \\
\hline Red meat & 15 & 34 & -- & -- & -- \\
\hline Hay & $(100)$ & $(100)$ & 45 & 2 & 200 \\
\hline Grain & $(180)$ & $(180)$ & 90 & 0.8 & 0 \\
\hline Poultry & 1 & 34 & 90 & 0.8 & 0 \\
\hline Fish & 1 & 1 & -- & -- & -- \\
\hline Drinking water & 1 & 1 & -- & -- & -- \\
\hline
\end{tabular}

(a) Holdup is the time between harvest and consumption.

(b) Values in ( ) are the holdup in days between harvest and consumption by farm animals. 
Table A.2. Dietary Parameters Used in Dose Calculations

\begin{tabular}{|l|c|c|}
\hline \multirow{2}{*}{\multicolumn{1}{c|}{ Medium }} & \multicolumn{2}{c|}{ Consumption } \\
\cline { 2 - 3 } & $\begin{array}{c}\text { Maximally Exposed } \\
\text { Individual }\end{array}$ & Average Individual \\
\hline Leafy vegetables & $30 \mathrm{~kg} / \mathrm{y}$ & $15 \mathrm{~kg} / \mathrm{y}$ \\
\hline Other vegetables & $220 \mathrm{~kg} / \mathrm{y}$ & $140 \mathrm{~kg} / \mathrm{y}$ \\
\hline Fruit & $330 \mathrm{~kg} / \mathrm{y}$ & $64 \mathrm{~kg} / \mathrm{y}$ \\
\hline Grain & $80 \mathrm{~kg} / \mathrm{y}$ & $72 \mathrm{~kg} / \mathrm{y}$ \\
\hline Eggs & $30 \mathrm{~kg} / \mathrm{y}$ & $20 \mathrm{~kg} / \mathrm{y}$ \\
\hline Milk & $270 \mathrm{~L} / \mathrm{y}$ & $230 \mathrm{~L} / \mathrm{y}$ \\
\hline Red meat & $80 \mathrm{~kg} / \mathrm{y}$ & $70 \mathrm{~kg} / \mathrm{y}$ \\
\hline Poultry & $18 \mathrm{~kg} / \mathrm{y}$ & $8.5 \mathrm{~kg} / \mathrm{y}$ \\
\hline Fish & $40 \mathrm{~kg} / \mathrm{y}$ & - (a) $^{-}$ \\
\hline Drinking water & $730 \mathrm{~L} / \mathrm{y}$ & $440 \mathrm{~L} / \mathrm{y}$ \\
\hline
\end{tabular}

(a) Average individual consumption not identified; radiation doses were calculated based on estimated total annual catch of $15,000 \mathrm{~kg}(33,075 \mathrm{lb})$.

Table A.3. Residency Parameters Used in Dose Calculations

\begin{tabular}{|l|c|c|}
\hline \multirow{2}{*}{\multicolumn{1}{c|}{ Parameter }} & \multicolumn{2}{|c|}{ Exposure, h/y } \\
\cline { 2 - 3 } & $\begin{array}{c}\text { Maximally Exposed } \\
\text { Individual }\end{array}$ & Average Individual \\
\hline Ground contamination & 4,383 & 2,920 \\
\hline Air submersion & 8,766 & 8,766 \\
\hline Inhalation $^{(\mathrm{a})}$ & 8,766 & 8,766 \\
\hline
\end{tabular}

(a) Inhalation rates: adult $270 \mathrm{~cm}^{3} / \mathrm{s}$

Table A.4. Recreational Parameters Used in Dose Calculations

\begin{tabular}{|l|c|c|}
\hline \multirow{2}{*}{ Parameter } & \multicolumn{2}{|c|}{ Exposure, h/y $\mathbf{y}^{(\mathbf{a})}$} \\
\cline { 2 - 3 } & $\begin{array}{c}\text { Maximally Exposed } \\
\text { Individual }\end{array}$ & Average Individual \\
\hline Shoreline & 500 & 17 \\
\hline Boating & 100 & 5 \\
\hline Swimming & 100 & 10 \\
\hline
\end{tabular}

(a) Assumed river-water travel times from 100-N Area to the point of aquatic recreation were $8 \mathrm{~h}$ for the maximally exposed individual and $13 \mathrm{~h}$ for the average individual. Correspondingly lesser times were used for other locations 


\section{Appendix B}

Doses to Maximally Exposed Individual, 1993-2000 


\section{Appendix B}

\section{Doses to Maximally Exposed Individual, 1993-2000}
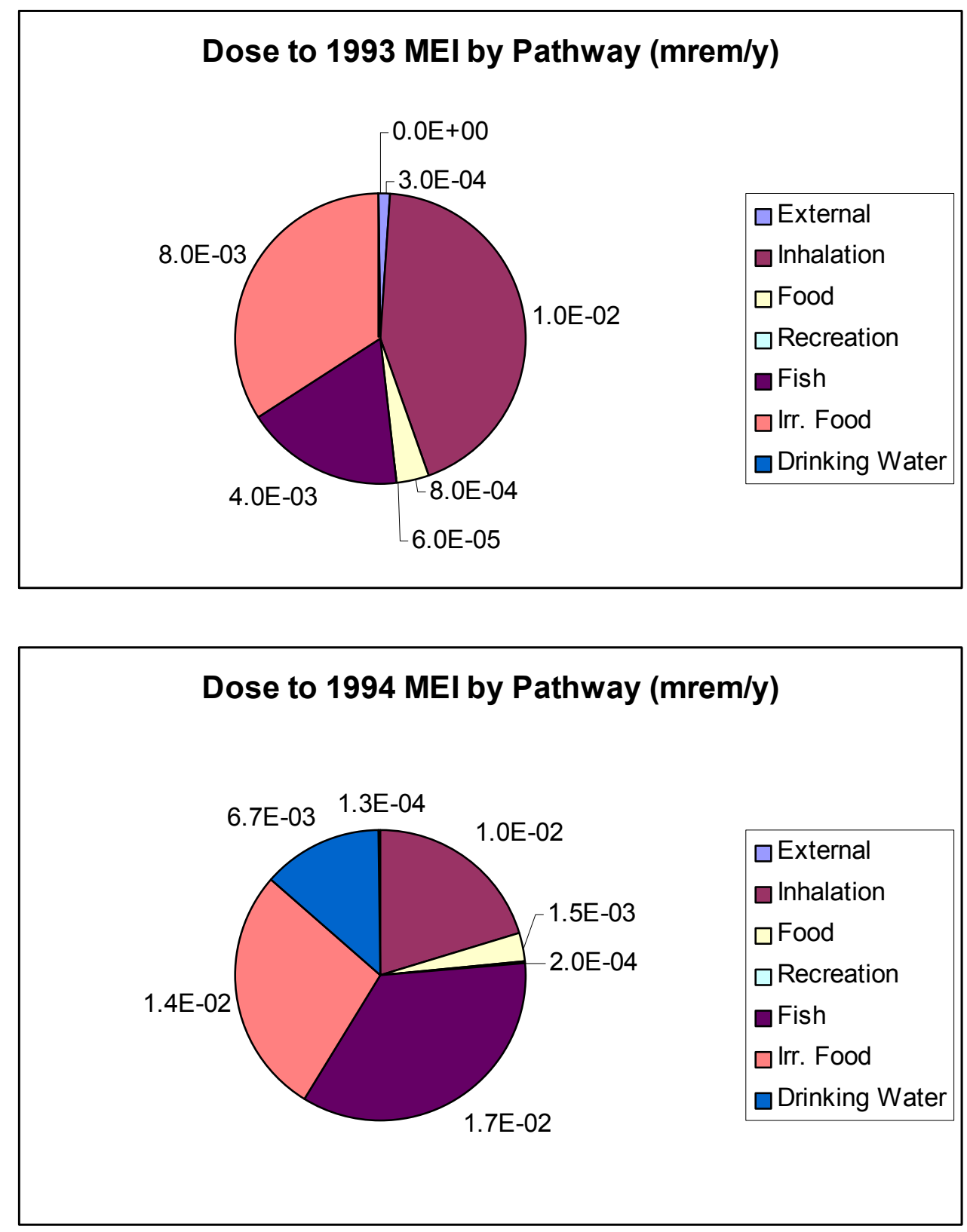

B. 1 

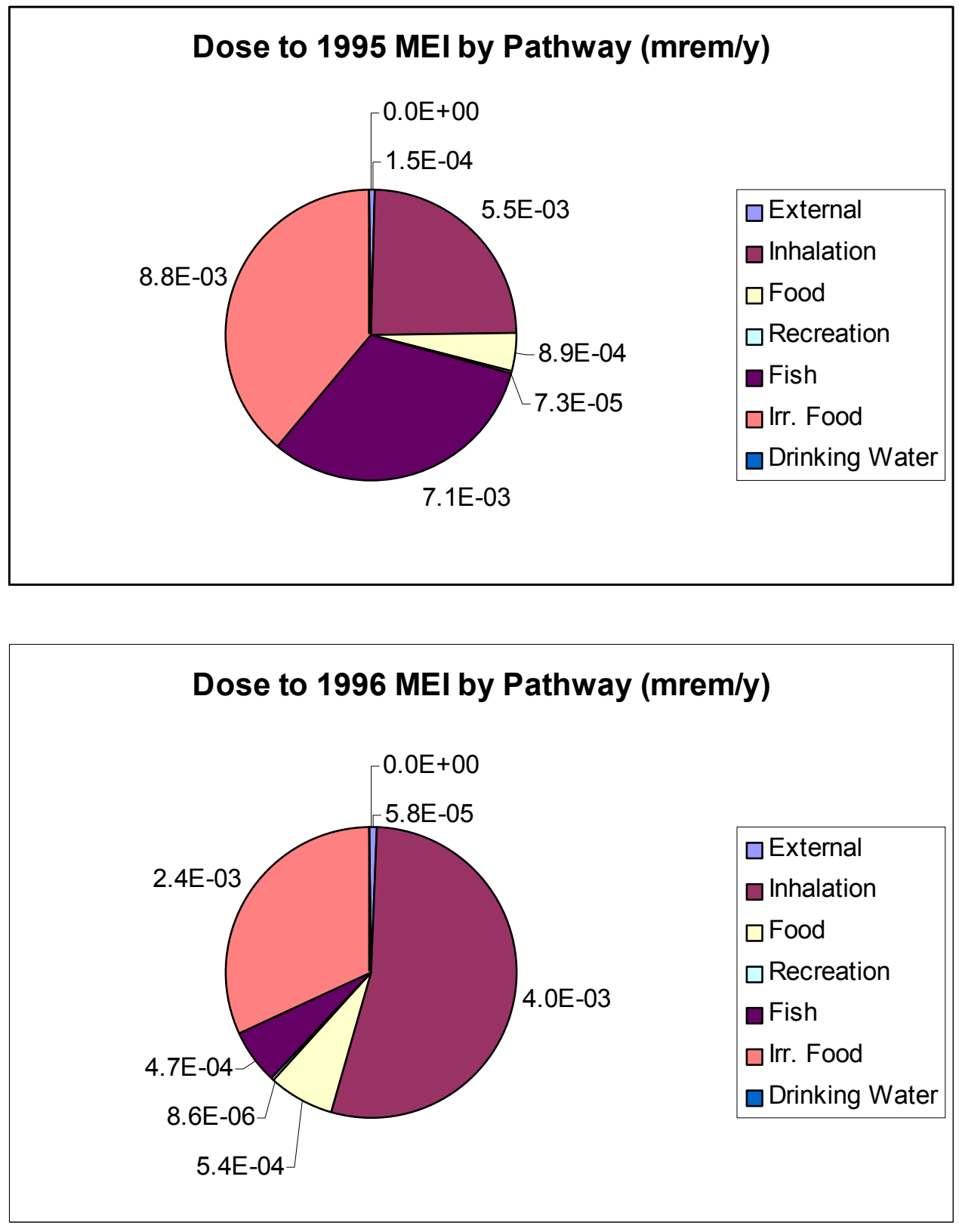


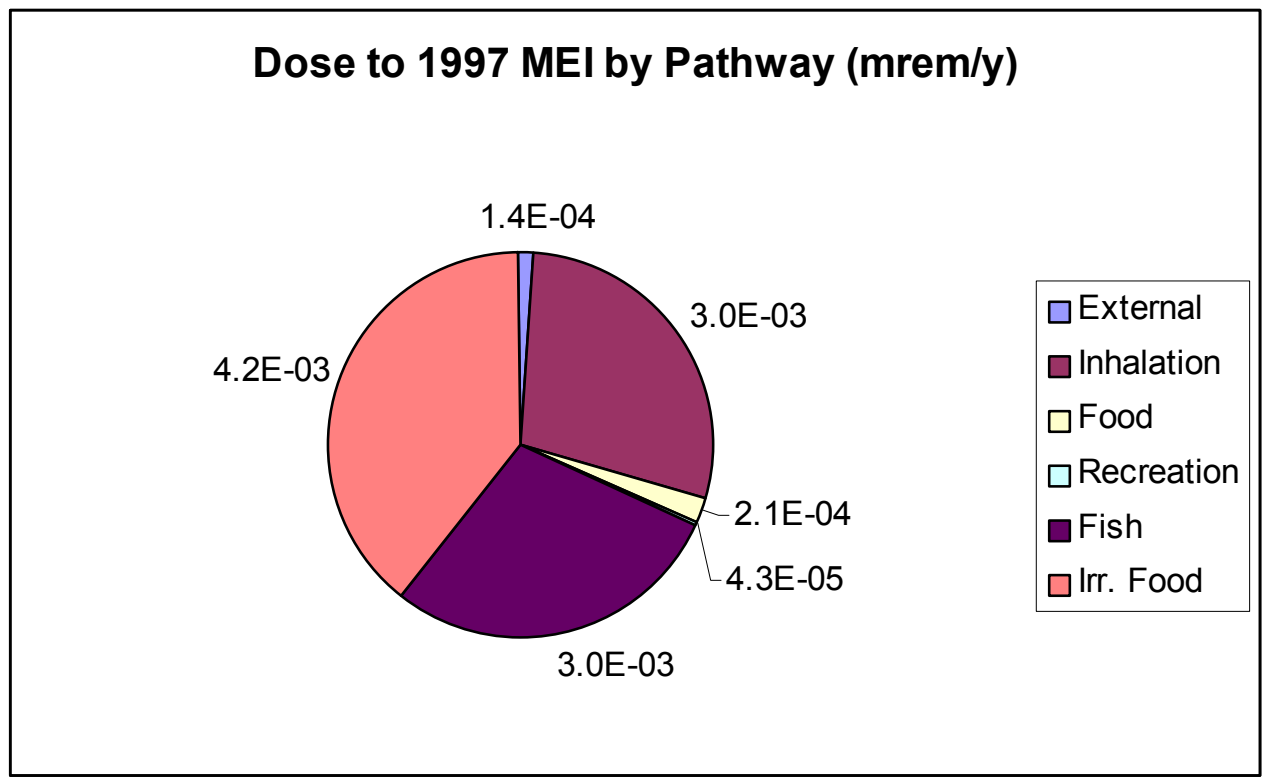

\section{Dose to 1998 MEI by Pathway (mrem/y)}

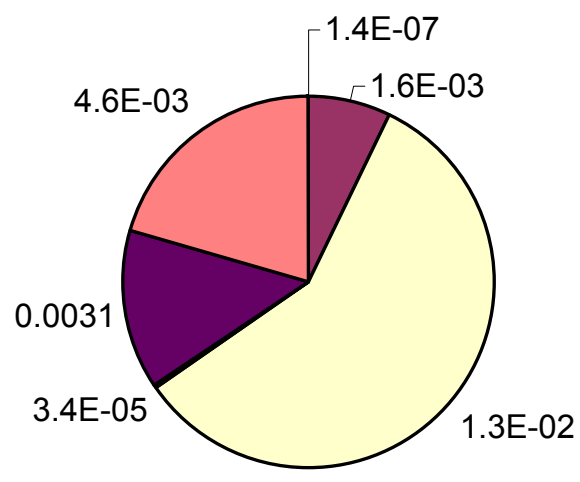

$\square$ External

口Inhalation

$\square$ Food

$\square$ Recreation

$\square$ Fish

DIrr. Food 

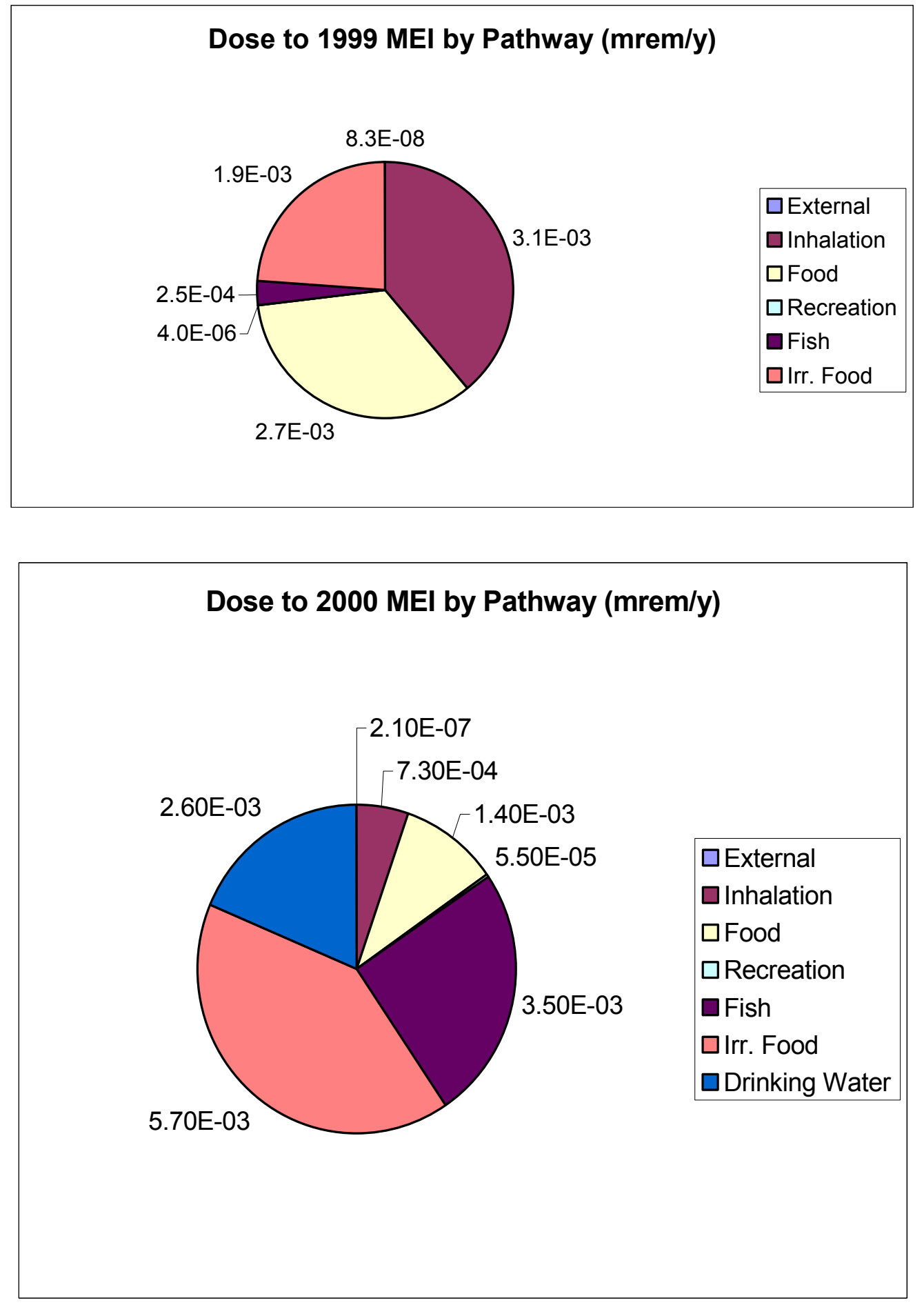

B. 4 


\section{Appendix C}

\section{Radionuclide Release Quantities,}

1993-2000 


\section{Appendix C}

\section{Radionuclide Release Quantities, 1993-2000}

Table C.1. Radionuclide Release Quantities (in Curies) by Operational Area for Years 1993 through 2000

\begin{tabular}{|c|c|c|c|c|c|c|c|c|c|}
\hline Area & Nuclide & 1993 & 1994 & 1995 & 1996 & 1997 & 1998 & 1999 & 2000 \\
\hline 100 & Am-241 & $5.4 \mathrm{E}-06$ & $5.6 \mathrm{E}-06$ & $5.7 \mathrm{E}-06$ & $2.0 \mathrm{E}-06$ & $2.5 \mathrm{E}-06$ & $2.0 \mathrm{E}-06$ & $2.40 \mathrm{E}-06$ & $2.6 \mathrm{E}-06$ \\
\hline 100 & Co-60 & $5.2 \mathrm{E}-06$ & $6.3 \mathrm{E}-06$ & $9.2 \mathrm{E}-06$ & $5.1 \mathrm{E}-07$ & $8.3 \mathrm{E}-10$ & & $3.90 \mathrm{E}-08$ & $3.4 \mathrm{E}-08$ \\
\hline 100 & $\mathrm{Cs} / \mathrm{Ba}-137$ & \begin{tabular}{|l|}
$1.6 \mathrm{E}-04$ \\
\end{tabular} & $1.0 \mathrm{E}-04$ & $2.5 \mathrm{E}-04$ & $5.1 \mathrm{E}-05$ & $5.5 \mathrm{E}-05$ & $3.0 \mathrm{E}-05$ & $4.54 \mathrm{E}-05$ & $1.1 \mathrm{E}-04$ \\
\hline 100 & Cs-134 & $8.9 \mathrm{E}-08$ & $3.2 \mathrm{E}-07$ & $1.3 \mathrm{E}-06$ & $1.3 \mathrm{E}-08$ & & & & \\
\hline 100 & Eu-154 & $6.3 \mathrm{E}-06$ & $1.7 \mathrm{E}-06$ & $8.3 \mathrm{E}-06$ & $4.5 \mathrm{E}-07$ & & & $4.80 \mathrm{E}-08$ & \\
\hline 100 & Eu-155 & $2.8 \mathrm{E}-06$ & $4.7 \mathrm{E}-06$ & $1.5 \mathrm{E}-06$ & $1.9 \mathrm{E}-07$ & & & & \\
\hline 100 & $\mathrm{Pu}-238$ & $1.0 \mathrm{E}-06$ & $1.2 \mathrm{E}-06$ & $2.3 \mathrm{E}-06$ & $5.2 \mathrm{E}-07$ & $5.8 \mathrm{E}-07$ & $5.2 \mathrm{E}-07$ & $5.80 \mathrm{E}-07$ & $8.4 \mathrm{E}-07$ \\
\hline 100 & $\mathrm{Pu}-239$ & $8.2 \mathrm{E}-06$ & \begin{tabular}{|l|}
$7.8 \mathrm{E}-06$ \\
\end{tabular} & $1.5 \mathrm{E}-05$ & $4.5 \mathrm{E}-06$ & $3.9 \mathrm{E}-06$ & $3.4 \mathrm{E}-06$ & $4.20 \mathrm{E}-06$ & 5.4E-06 \\
\hline 100 & $\mathrm{Pu}-241$ & & & $2.1 \mathrm{E}-04$ & $4.1 \mathrm{E}-05$ & $4.0 \mathrm{E}-05$ & $3.8 \mathrm{E}-05$ & $5.10 \mathrm{E}-05$ & $6.8 \mathrm{E}-05$ \\
\hline 100 & Ru-106 & \begin{tabular}{|l|}
$1.3 \mathrm{E}-05$ \\
\end{tabular} & $1.4 \mathrm{E}-05$ & $1.1 \mathrm{E}-05$ & $5.4 \mathrm{E}-07$ & & & & \\
\hline 100 & Sb-125 & $6.0 \mathrm{E}-06$ & $1.6 \mathrm{E}-06$ & $2.8 \mathrm{E}-06$ & $1.9 \mathrm{E}-07$ & $3.7 \mathrm{E}-09$ & & $5.00 \mathrm{E}-08$ & \\
\hline 100 & Sr-90 & \begin{tabular}{|l|}
$5.4 \mathrm{E}-05$ \\
\end{tabular} & $4.7 \mathrm{E}-05$ & $6.1 \mathrm{E}-05$ & $2.8 \mathrm{E}-05$ & $2.1 \mathrm{E}-05$ & $1.7 \mathrm{E}-05$ & $1.90 \mathrm{E}-05$ & $4.1 \mathrm{E}-05$ \\
\hline $200 \mathrm{E}$ & Am-241 & $2.8 \mathrm{E}-05$ & $1.2 \mathrm{E}-05$ & $1.6 \mathrm{E}-05$ & $9.2 \mathrm{E}-06$ & $4.8 \mathrm{E}-06$ & \begin{tabular}{|l|}
$5.0 \mathrm{E}-07$ \\
\end{tabular} & $5.60 \mathrm{E}-07$ & $4.8 \mathrm{E}-06$ \\
\hline $200 \mathrm{E}$ & Co-60 & & & & $7.7 \mathrm{E}-10$ & & & $1.60 \mathrm{E}-09$ & \\
\hline $200 \mathrm{E}$ & $\mathrm{Cs} / \mathrm{Ba}-137$ & $1.5 \mathrm{E}-03$ & $2.9 \mathrm{E}-04$ & $3.8 \mathrm{E}-04$ & $5.5 \mathrm{E}-04$ & $9.1 \mathrm{E}-04$ & $1.9 \mathrm{E}-04$ & $3.90 \mathrm{E}-05$ & $6.7 \mathrm{E}-05$ \\
\hline $200 \mathrm{E}$ & Cs-134 & & & $2.4 \mathrm{E}-08$ & $3.0 \mathrm{E}-09$ & & & & \\
\hline $200 \mathrm{E}$ & Eu-152 & & & $3.7 \mathrm{E}-07$ & & & & & \\
\hline $200 \mathrm{E}$ & Eu-154 & & & $4.6 \mathrm{E}-07$ & & & & & \\
\hline $200 \mathrm{E}$ & $\mathrm{Eu}-155$ & & & $2.2 \mathrm{E}-07$ & & & & & \\
\hline $200 \mathrm{E}$ & I-129 & $4.8 \mathrm{E}-03$ & $1.4 \mathrm{E}-02$ & $8.9 \mathrm{E}-03$ & $3.9 \mathrm{E}-03$ & $1.4 \mathrm{E}-03$ & $3.1 \mathrm{E}-04$ & $1.90 \mathrm{E}-04$ & $1.2 \mathrm{E}-03$ \\
\hline $200 \mathrm{E}$ & $\mathrm{Pb}-212$ & $9.7 \mathrm{E}-04$ & & & & & & & \\
\hline $200 \mathrm{E}$ & Pm-147 & $1.1 \mathrm{E}-04$ & & & & & & & \\
\hline $200 \mathrm{E}$ & $\mathrm{Pu}-238$ & $3.2 \mathrm{E}-06$ & & $6.9 \mathrm{E}-07$ & $2.2 \mathrm{E}-07$ & $1.8 \mathrm{E}-07$ & $7.9 \mathrm{E}-10$ & $1.90 \mathrm{E}-08$ & $9.8 \mathrm{E}-08$ \\
\hline $200 \mathrm{E}$ & $\mathrm{Pu}-239$ & $1.1 \mathrm{E}-05$ & $3.2 \mathrm{E}-05$ & 7.9E-06 & $6.7 \mathrm{E}-06$ & $6.3 \mathrm{E}-06$ & $1.1 \mathrm{E}-06$ & $6.90 \mathrm{E}-07$ & $2.5 \mathrm{E}-06$ \\
\hline $200 \mathrm{E}$ & $\mathrm{Pu}-241$ & $3.3 \mathrm{E}-05$ & $1.8 \mathrm{E}-04$ & $1.2 \mathrm{E}-04$ & $1.7 \mathrm{E}-05$ & 6.4E-06 & $2.9 \mathrm{E}-08$ & $1.20 \mathrm{E}-06$ & $6.1 \mathrm{E}-05$ \\
\hline $200 \mathrm{E}$ & Rn-220 & $1.2 \mathrm{E}+01$ & & & & & & & \\
\hline $200 \mathrm{E}$ & Ru-106 & & & $6.7 \mathrm{E}-06$ & 9.5E-08 & & & & \\
\hline $200 \mathrm{E}$ & Sb-125 & & & $9.1 \mathrm{E}-06$ & $2.0 \mathrm{E}-06$ & & $4.8 \mathrm{E}-07$ & & $1.8 \mathrm{E}-06$ \\
\hline $200 \mathrm{E}$ & Sn-113 & & $2.2 \mathrm{E}-06$ & $8.0 \mathrm{E}-07$ & & & & & \\
\hline $200 \mathrm{E}$ & Sr-90 & \begin{tabular}{|l|}
$1.4 \mathrm{E}-04$ \\
\end{tabular} & $6.5 \mathrm{E}-05$ & $6.8 \mathrm{E}-05$ & $6.2 \mathrm{E}-05$ & $2.5 \mathrm{E}-04$ & $1.2 \mathrm{E}-04$ & $9.50 \mathrm{E}-05$ & $9.1 \mathrm{E}-05$ \\
\hline $200 \mathrm{~W}$ & Am-241 & 9.9E-05 & $6.6 \mathrm{E}-05$ & $1.7 \mathrm{E}-05$ & $3.7 \mathrm{E}-05$ & $2.0 \mathrm{E}-05$ & $3.0 \mathrm{E}-05$ & $4.50 \mathrm{E}-05$ & $8.7 \mathrm{E}-05$ \\
\hline $200 \mathrm{~W}$ & $\mathrm{Cs} / \mathrm{Ba}-137$ & $2.3 \mathrm{E}-04$ & $2.7 \mathrm{E}-04$ & $1.9 \mathrm{E}-05$ & $6.5 \mathrm{E}-07$ & 7.7E-09 & $3.2 \mathrm{E}-09$ & $2.50 \mathrm{E}-09$ & $2.1 \mathrm{E}-09$ \\
\hline $200 \mathrm{~W}$ & Cs-134 & & & $1.1 \mathrm{E}-07$ & & & & & \\
\hline
\end{tabular}


Table C.1. (Contd)

\begin{tabular}{|c|c|c|c|c|c|c|c|c|c|}
\hline Area & Nuclide & 1993 & 1994 & 1995 & 1996 & 1997 & 1998 & 1999 & 2000 \\
\hline $200 \mathrm{~W}$ & Eu-152 & & & $1.6 \mathrm{E}-07$ & & & & & \\
\hline $200 W$ & Eu-154 & & & $2.6 \mathrm{E}-07$ & & & & & \\
\hline $200 \mathrm{~W}$ & Eu-155 & & & $1.2 \mathrm{E}-07$ & & & & & \\
\hline $200 \mathrm{~W}$ & $\mathrm{Pu}-238$ & & $1.5 \mathrm{E}-05$ & $2.6 \mathrm{E}-06$ & 4.2E-06 & 2.2E-06 & $3.4 \mathrm{E}-06$ & 4.90E-06 & $1.1 \mathrm{E}-05$ \\
\hline $200 \mathrm{~W}$ & Pu-239 & $5.5 \mathrm{E}-04$ & $3.9 \mathrm{E}-04$ & $1.0 \mathrm{E}-04$ & $2.4 \mathrm{E}-04$ & $1.1 \mathrm{E}-04$ & $2.0 \mathrm{E}-04$ & $2.10 \mathrm{E}-04$ & $5.1 \mathrm{E}-04$ \\
\hline $200 W$ & $\mathrm{Pu}-241$ & $3.4 \mathrm{E}-03$ & $2.0 \mathrm{E}-03$ & $2.0 \mathrm{E}-04$ & $3.5 \mathrm{E}-04$ & $4.6 \mathrm{E}-05$ & $4.4 \mathrm{E}-05$ & $1.20 \mathrm{E}-04$ & $3.1 \mathrm{E}-04$ \\
\hline $200 \mathrm{~W}$ & Ru-106 & $4.0 \mathrm{E}-06$ & & $1.0 \mathrm{E}-08$ & & & & & \\
\hline $200 W$ & Sb-125 & & & $1.2 \mathrm{E}-07$ & & & & & \\
\hline $200 W$ & Sn-113 & & & $1.4 \mathrm{E}-07$ & & & & & \\
\hline $200 \mathrm{~W}$ & Sr-90 & $1.3 \mathrm{E}-04$ & $7.5 \mathrm{E}-05$ & $1.0 \mathrm{E}-04$ & 3.6E-04 & $3.0 \mathrm{E}-04$ & $2.3 \mathrm{E}-04$ & 2.90E-04 & $1.9 \mathrm{E}-04$ \\
\hline $200 \mathrm{~W}$ & U-234 & $9.5 \mathrm{E}-06$ & $4.0 \mathrm{E}-07$ & & & & & & \\
\hline $200 \mathrm{~W}$ & U-235 & $3.4 \mathrm{E}-07$ & $1.4 \mathrm{E}-08$ & & & & & & \\
\hline $200 W$ & U-236 & $6.8 \mathrm{E}-07$ & $3.3 \mathrm{E}-08$ & & & & & & \\
\hline $200 \mathrm{~W}$ & U-238 & $6.5 \mathrm{E}-06$ & $2.4 \mathrm{E}-07$ & & & & & & \\
\hline 300 & Am-241 & $5.5 \mathrm{E}-08$ & $6.7 \mathrm{E}-08$ & $1.1 \mathrm{E}-08$ & 7.0E-08 & $6.5 \mathrm{E}-09$ & 2.3E-08 & $1.40 \mathrm{E}-07$ & $3.4 \mathrm{E}-08$ \\
\hline 300 & Co-60 & $1.4 \mathrm{E}-08$ & $8.6 \mathrm{E}-08$ & & & & & & \\
\hline 300 & $\mathrm{Cs} / \mathrm{Ba}-137$ & $9.3 \mathrm{E}-07$ & 7.2E-06 & $1.5 \mathrm{E}-06$ & $3.3 \mathrm{E}-06$ & 7.9E-07 & $5.8 \mathrm{E}-07$ & $4.23 \mathrm{E}-07$ & $1.6 \mathrm{E}-06$ \\
\hline 300 & Cs-134 & $3.3 \mathrm{E}-07$ & & $2.7 \mathrm{E}-08$ & & & & & \\
\hline 300 & Eu-154 & $1.5 \mathrm{E}-06$ & $2.7 \mathrm{E}-07$ & & & & & & \\
\hline 300 & Eu-155 & $2.6 \mathrm{E}-08$ & & $4.3 \mathrm{E}-08$ & & & & & \\
\hline 300 & H-3 (HT) & $1.0 \mathrm{E}+01$ & $1.0 \mathrm{E}+01$ & $3.8 \mathrm{E}+00$ & $1.7 \mathrm{E}+00$ & $2.1 \mathrm{E}+01$ & $1.1 \mathrm{E}+02$ & $4.00 \mathrm{E}+01$ & $4.3 \mathrm{E}+01$ \\
\hline 300 & H-3 (HTO) & $1.1 \mathrm{E}+01$ & $1.7 \mathrm{E}+00$ & $2.8 \mathrm{E}+00$ & $1.8 \mathrm{E}+00$ & $1.5 \mathrm{E}+00$ & $1.7 \mathrm{E}+02$ & $1.50 \mathrm{E}+02$ & $7.9 \mathrm{E}+01$ \\
\hline 300 & I-129 & & & & & & 4.6E-08 & & \\
\hline 300 & $\mathrm{Kr}-85$ & & $4.1 \mathrm{E}+00$ & & & & & & \\
\hline 300 & $\mathrm{Pu}-238$ & 6.9E-08 & $2.1 \mathrm{E}-08$ & $2.5 \mathrm{E}-09$ & 1.9E-08 & $9.5 \mathrm{E}-10$ & $1.7 \mathrm{E}-09$ & & 7.6E-09 \\
\hline 300 & $\mathrm{Pu}-239$ & 7.0E-06 & $3.6 \mathrm{E}-06$ & $2.1 \mathrm{E}-06$ & 1.9E-06 & $1.1 \mathrm{E}-06$ & $1.1 \mathrm{E}-06$ & $1.12 \mathrm{E}-06$ & $8.2 \mathrm{E}-07$ \\
\hline 300 & $\mathrm{Rn}-220$ & $8.5 \mathrm{E}+01$ & $2.3 \mathrm{E}-01$ & $7.9 \mathrm{E}+01$ & $5.4 \mathrm{E}+01$ & $5.0 \mathrm{E}+01$ & & & \\
\hline 300 & $\mathrm{Rn}-222$ & $1.5 \mathrm{E}+00$ & $1.2 \mathrm{E}+00$ & 4.0E-01 & $5.0 \mathrm{E}-01$ & $1.6 \mathrm{E}+00$ & & & \\
\hline 300 & Ru-106 & & $2.4 \mathrm{E}-06$ & & & & & & \\
\hline 300 & Sb-125 & $1.5 \mathrm{E}-06$ & & & & & & $1.10 \mathrm{E}-07$ & \\
\hline 300 & Sr-90 & $4.3 \mathrm{E}-05$ & $1.6 \mathrm{E}-05$ & $1.8 \mathrm{E}-05$ & $2.0 \mathrm{E}-05$ & $1.5 \mathrm{E}-05$ & $9.6 \mathrm{E}-06$ & $1.01 \mathrm{E}-05$ & $1.0 \mathrm{E}-05$ \\
\hline 300 & Tc-99 & & & & & & & & $1.7 \mathrm{E}-08$ \\
\hline 300 & U-234 & 5.9E-09 & $1.2 \mathrm{E}-08$ & 7.9E-09 & & & & & \\
\hline 300 & U-235 & $6.2 \mathrm{E}-10$ & $2.9 \mathrm{E}-10$ & $1.8 \mathrm{E}-10$ & & & & & \\
\hline 300 & U-236 & & $1.8 \mathrm{E}-10$ & & & & & & \\
\hline 300 & U-238 & $5.9 \mathrm{E}-09$ & $1.7 \mathrm{E}-08$ & $1.4 \mathrm{E}-08$ & & & & & \\
\hline 300 & $\mathrm{Zn}-65$ & & $7.1 \mathrm{E}-07$ & & & & & & \\
\hline 300 & Zr-95 & & $1.1 \mathrm{E}-06$ & & & & & & \\
\hline 400 & $\mathrm{Cs} / \mathrm{Ba}-137$ & $8.2 \mathrm{E}-06$ & $7.0 \mathrm{E}-06$ & $5.9 \mathrm{E}-06$ & $5.5 \mathrm{E}-06$ & $4.6 \mathrm{E}-06$ & $5.5 \mathrm{E}-06$ & $1.90 \mathrm{E}-06$ & $3.5 \mathrm{E}-06$ \\
\hline 400 & H-3 (HTO) & $2.1 \mathrm{E}+00$ & & $2.5 \mathrm{E}-02$ & $3.6 \mathrm{E}+00$ & $7.9 \mathrm{E}+00$ & $4.2 \mathrm{E}+00$ & $1.4 \mathrm{E}+00$ & $8.8 \mathrm{E}-01$ \\
\hline 400 & $\mathrm{Pu}-239$ & $2.4 \mathrm{E}-06$ & $2.3 \mathrm{E}-06$ & $1.7 \mathrm{E}-06$ & $8.3 \mathrm{E}-07$ & $3.8 \mathrm{E}-07$ & $5.0 \mathrm{E}-07$ & $3.00 \mathrm{E}-07$ & $3.0 \mathrm{E}-07$ \\
\hline 400 & Sr-90 & & $6.7 \mathrm{E}-08$ & $6.1 \mathrm{E}-08$ & 9.0E-09 & & & & \\
\hline
\end{tabular}




\section{Appendix D}

Standard Assumptions Used for Hanford Site Dose Assessments 


\section{Appendix D}

\section{Standard Assumptions Used for Hanford Site Dose Assessments}

Table D.1. Hanford-Specific Parameters Recommended for Use in Calculating Dose to Maximally Exposed Receptors from Radionuclide Air Emissions

\begin{tabular}{|c|c|c|c|c|c|}
\hline \multirow[b]{2}{*}{ Parameter } & \multirow[b]{2}{*}{ Units } & \multirow{2}{*}{$\begin{array}{l}\text { CAP88-PC } \\
\text { Default }\end{array}$} & \multicolumn{2}{|c|}{ Recommended Values } & \multirow{2}{*}{$\begin{array}{c}\text { Basis for Hanford } \\
\text { Recommended Values }\end{array}$} \\
\hline & & & MEI & Average & \\
\hline \multicolumn{6}{|c|}{ Food Production Parameters } \\
\hline $\begin{array}{l}\text { Fraction of } \\
\text { Radioactivity on Veg. } \\
\& \text { Prod. after Washing }\end{array}$ & - & 0.50 & 1.00 & 1.00 & Conservative standard assumption \\
\hline $\begin{array}{l}\text { Fraction Year Animals } \\
\text { Graze on Pasture }\end{array}$ & - & 0.40 & 0.75 & 0.75 & $\begin{array}{l}\text { Schreckhise et al. 1993, } \\
\text { local growing season }\end{array}$ \\
\hline $\begin{array}{l}\text { Fraction Animals Daily } \\
\text { Feed is Pasture Grass }\end{array}$ & - & 0.43 & 1.0 & 1.0 & $\begin{array}{l}\text { Schreckhise et al. 1993, } \\
\text { local practice }\end{array}$ \\
\hline $\begin{array}{l}\text { Removal Rate Constant } \\
\text { - Physical Loss by } \\
\text { Weathering }\end{array}$ & hour $^{-1}$ & $2.9 \mathrm{E}-03$ & $3.0 \mathrm{E}-03$ & $3.0 \mathrm{E}-03$ & Schreckhise et al. 1993 \\
\hline $\begin{array}{l}\text { Fallout Interception } \\
\text { Fraction-Pasture }\end{array}$ & - & 0.57 & 0.25 & 0.25 & Schreckhise et al. 1993 \\
\hline $\begin{array}{l}\text { Fallout Interception } \\
\text { Fraction-Vegetable }\end{array}$ & - & 0.20 & 0.25 & 0.25 & Schreckhise et al. 1993 \\
\hline $\begin{array}{l}\text { Period Exposure - } \\
\text { Growing Season - } \\
\text { Crops/Leafy Veg. }\end{array}$ & hours & 1440. & 2160. & 2160. & $\begin{array}{l}\text { Schreckhise et al. 1993, } \\
\text { Napier 1981, McCormack et al. } \\
1984, \\
\text { local growing season }\end{array}$ \\
\hline $\begin{array}{l}\text { Time Delay - Ingestion } \\
\text { Stored Feed }\end{array}$ & hours & 2160. & 2400. & 2400. & Schreckhise et al. 1993 \\
\hline $\begin{array}{l}\text { Time Delay - Ingestion } \\
\text { Leafy Veg. - Man }\end{array}$ & hours & 336. & 24. & 336. & $\begin{array}{l}\text { Schreckhise et al. 1993, } \\
\text { Napier 1981, McCormack et al. } \\
1984\end{array}$ \\
\hline $\begin{array}{l}\text { Time Delay - Ingestion } \\
\text { Produce - Man }\end{array}$ & hours & 336. & 120. & 336. & $\begin{array}{l}\text { Schreckhise et al. } 1993 \\
\text { (vegetables other than leafy) }\end{array}$ \\
\hline $\begin{array}{l}\text { Avg. Time - Slaughter } \\
\text { to Consumption }\end{array}$ & days & 20. & 15. & 34. & $\begin{array}{l}\text { Schreckhise et al. 1993, } \\
\text { Napier 1981, McCormack et al. } \\
1984\end{array}$ \\
\hline $\begin{array}{l}\text { Agr. Productivity by } \\
\text { Unit Area - Milk } \\
\text { (Pasture) }\end{array}$ & $\begin{array}{l}\mathrm{kg} / \mathrm{m}^{2} \\
\text { (dry) }\end{array}$ & 0.28 & 0.30 & 0.30 & $\begin{array}{l}\text { Schreckhise et al. } 1993 \\
\text { (pasture yield x dry/wet ratio) }\end{array}$ \\
\hline $\begin{array}{l}\text { Agr. Productivity by } \\
\text { Unit Area - Prod/Leafy } \\
\text { Veg. }\end{array}$ & $\begin{array}{l}\mathrm{kg} / \mathrm{m}^{2} \\
\text { (fresh) }\end{array}$ & 0.716 & 2.0 & 2.0 & $\begin{array}{l}\text { Schreckhise et al. 1993, } \\
\text { McCormack et al. } 1984 \\
\text { (leafy vegetables) }\end{array}$ \\
\hline $\begin{array}{l}\text { Effec. Surface Density } \\
\text { of Soil, Dry Weight }\end{array}$ & $\mathrm{kg} / \mathrm{m}^{2}$ & 215.0 & 224.0 & 224.0 & Schreckhise et al. 1993 \\
\hline
\end{tabular}


Table D.1. (Contd)

\begin{tabular}{|c|c|c|c|c|c|}
\hline \multirow[b]{2}{*}{ Parameter } & \multirow[b]{2}{*}{ Units } & \multirow{2}{*}{$\begin{array}{l}\text { CAP88-PC } \\
\text { Default }\end{array}$} & \multicolumn{2}{|c|}{ Recommended Values } & \multirow{2}{*}{$\begin{array}{c}\text { Basis for Hanford } \\
\text { Recommended Values }\end{array}$} \\
\hline & & & MEI & Average & \\
\hline \multicolumn{6}{|c|}{ Food Consumption Parameters } \\
\hline $\begin{array}{l}\text { Ingestion Rate of Meat } \\
\text { by Man }\end{array}$ & $\mathrm{kg} /$ year & 85. & 98. & 79. & $\begin{array}{l}\text { Schreckhise et al. 1993, } \\
\text { Napier 1981, McCormack et al. } \\
1984 \\
\text { (sum of beef, pork, poultry) }\end{array}$ \\
\hline $\begin{array}{l}\text { Ingestion Rate of Leafy } \\
\text { Veg. By Man }\end{array}$ & $\mathrm{kg} /$ year & 18. & 30. & 15. & $\begin{array}{l}\text { Schreckhise et al. 1993, } \\
\text { Napier 1981, McCormack et al. } \\
1984\end{array}$ \\
\hline $\begin{array}{l}\text { Ingestion Rate of Milk } \\
\text { by Man }\end{array}$ & L/year & 112. & 270. & 230. & $\begin{array}{l}\text { Schreckhise et al. 1993, } \\
\text { Napier 1981, McCormack et al. } \\
1984\end{array}$ \\
\hline $\begin{array}{l}\text { Ingestion Rate of } \\
\text { Produce by Man }\end{array}$ & $\mathrm{kg} /$ year & 176. & 220. & 140. & $\begin{array}{l}\text { Schreckhise et al. } 1993 \\
\text { (vegetables other than leafy) }\end{array}$ \\
\hline $\begin{array}{l}\text { Fraction Produce } \\
\text { Ingested From Garden } \\
\text { of Interest }\end{array}$ & - & 1.0 & 1.0 & 0.25 & EPA 1989 \\
\hline $\begin{array}{l}\text { Fraction Leafy Veg. } \\
\text { Ingested From Garden } \\
\text { of Interest }\end{array}$ & - & 1.0 & 1.0 & 0.25 & EPA 1989 \\
\hline \multicolumn{6}{|c|}{ Other Exposure Parameters } \\
\hline $\begin{array}{l}\text { Ground Surface } \\
\text { Correction Factor }\end{array}$ & - & 0.50 & 1.0 & 0.50 & $\begin{array}{l}\text { MEI uses original AIRDOS/CAP- } \\
88 \text { default. Appropriate for } \\
\text { relatively flat, unvegetated, arid } \\
\text { site }\end{array}$ \\
\hline $\begin{array}{l}\text { Fraction Time Spent } \\
\text { Swimming }\end{array}$ & - & 0.0 & $1.0 \mathrm{E}-02$ & $\mathrm{~N} / \mathrm{A}$ & $\begin{array}{l}\text { Not used in calculation by updated } \\
\text { code - no effect. }\end{array}$ \\
\hline Inhalation Rate of Man & $\mathrm{cm}^{3} / \mathrm{h}$ & $9.17 \mathrm{E}+05$ & $9.7 \mathrm{E}+05$ & $9.7 \mathrm{E}+05$ & $\begin{array}{l}\text { ICRP } 23 \text { (1975) daily average } \\
\text { value for reference man }\end{array}$ \\
\hline $\begin{array}{l}\text { Period Long-term } \\
\text { Buildup in Soil }\end{array}$ & years & 100.0 & $\begin{array}{l}50.0 \text { or as } \\
\text { appropriate for } \\
\text { emission unit }\end{array}$ & $\begin{array}{l}50.0 \text { or as } \\
\text { appropriate for } \\
\text { emission unit }\end{array}$ & $\begin{array}{l}\text { Expected maximum operating life } \\
\text { for Hanford facilities based on end } \\
\text { of most site operations by } 2046 \text {. } \\
\text { Original AIRDOS/CAP- } 88 \text { default } \\
\text { was } 1 \text { year. }\end{array}$ \\
\hline $\begin{array}{l}\text { Hanford Site Population } \\
\text { within } 50 \mathrm{mi}(80 \mathrm{~km})\end{array}$ & people & $\mathrm{N} / \mathrm{A}$ & \begin{tabular}{|l|} 
Specific to \\
Emissions Source
\end{tabular} & \begin{tabular}{|l|} 
Specific to \\
Emissions Source
\end{tabular} & Beck et al. 1991 \\
\hline $\begin{array}{l}\text { Hanford Site } \\
\text { Meteorological Data }\end{array}$ & $\begin{array}{l}\text { Joint frequency } \\
\text { of wind speed, } \\
\text { direction, and } \\
\text { atmospheric } \\
\text { stability }\end{array}$ & $\mathrm{N} / \mathrm{A}$ & \begin{tabular}{|l|} 
Specific to \\
Emissions Source
\end{tabular} & \begin{tabular}{|l|} 
Specific to \\
Emissions Source
\end{tabular} & $\begin{array}{l}\text { Meteorological data collected } \\
\text { according to methods described in } \\
\text { Hoitink et al. } 2000 .\end{array}$ \\
\hline
\end{tabular}




\section{D.1 References}

Beck, D. M., B. A. Napier, M. J. Scott, A. G. Thurman, M. D. Davis, D. B. Pittenger, S. F. Shindle, and N. C. Batishko. 1991. Hanford Area 1990 Population and 50-year Projections. PNL-7803, Pacific Northwest Laboratory, Richland, Washington.

Hoitink, D. J., K. W. Burk, and J. V. Ramsdell. 2000. Hanford Site Climatological Data Summary Calendar Year 1999 with Historical Data. PNNL-13117, Pacific Northwest National Laboratory, Richland, Washington.

International Commission on Radiological Protection (ICRP). 1975. Report of the Task Group on Reference Man. ICRP, Publication Number 23, Pergamon Press, Oxford.

McCormack, W. D., J. V. Ramsdell and B. A. Napier. 1984. Hanford Dose Overview Program:

Standardized Methods and Data for Hanford Environmental Dose Calculations. PNL-3777, Rev. 1. Pacific Northwest Laboratory, Richland, Washington.

Napier, B. A. 1981. Standardized Input for Hanford Environmental Impact Statements, Part 1. PNL-3509, Pt. 1. Pacific Northwest Laboratory, Richland, Washington.

Schreckhise, R. G., K. Rhoads, J. S. Davis, B. A. Napier, and J. V. Ramsdell. 1993. Recommended Environmental Dose Calculation Methods and Hanford-Specific Parameters. PNL-3777, Rev. 2. Pacific Northwest Laboratory, Richland, Washington.

U.S. Environmental Protection Agency (EPA). 1989. Exposure Factors Handbook. EPA/600/8-89/043, Washington, D.C. 


\section{Appendix E}

Comparison of Onsite Worker to Offsite Resident Doses for $200 \mathrm{~W}$ Area 


\section{Appendix E}

\section{Comparison of Onsite Worker to Offsite Resident Doses for $200 \mathrm{~W}$ Area}

Table E.1. 200 West Area-LIGO Worker CAP88-PC Unit Dose Factors (mrem/y per Ci/y) by Effective Release Height with Regional Ingestion ${ }^{(a)}$ (Released at 18310 m ESE)

\begin{tabular}{|c|c|c|c|c|c|c|}
\hline \multirow[b]{3}{*}{ Nuclide } & \multicolumn{3}{|c|}{ 10-m Release Height } & \multicolumn{3}{|c|}{ 40-m Release Height } \\
\hline & \multicolumn{3}{|c|}{$1.846 \mathrm{E}-07 \mathrm{~s} / \mathrm{m}^{3} \mathrm{CHI} / \mathrm{Q}$} & \multicolumn{3}{|c|}{ 8.982E-08 s $/ \mathrm{m}^{3} \mathrm{CHI} / \mathrm{Q}$} \\
\hline & $\begin{array}{l}\text { Onsite } 2000 \mathrm{~h} / \mathbf{y}+ \\
\text { regional ingestion }\end{array}$ & $\begin{array}{c}\text { Offsite Resident } \\
(\mathrm{mrem} / \mathrm{y})\end{array}$ & $\begin{array}{c}\text { Onsite }> \\
\text { Offsite }\end{array}$ & $\begin{array}{l}\text { Onsite } 2000 \mathrm{~h} / \mathrm{y}+ \\
\text { regional ingestion }\end{array}$ & $\begin{array}{c}\text { Offsite Resident } \\
(\mathrm{mrem} / \mathrm{y})\end{array}$ & Onsite $>$ Offsite \\
\hline $\mathrm{H}-3$ & $5.76 \mathrm{E}-06$ & $2.50 \mathrm{E}-05$ & & $3.15 \mathrm{E}-06$ & $1.40 \mathrm{E}-05$ & \\
\hline $\mathrm{Be}-7$ & $5.76 \mathrm{E}-05$ & $1.50 \mathrm{E}-04$ & & $3.95 \mathrm{E}-05$ & $1.20 \mathrm{E}-04$ & \\
\hline $\mathrm{C}-11$ & $1.59 \mathrm{E}-07$ & $3.80 \mathrm{E}-07$ & & $1.23 \mathrm{E}-07$ & $3.40 \mathrm{E}-07$ & \\
\hline $\mathrm{C}-14$ & $2.98 \mathrm{E}-04$ & $2.00 \mathrm{E}-03$ & & $1.69 \mathrm{E}-04$ & $1.10 \mathrm{E}-03$ & \\
\hline C-15 (b) & $0.00 \mathrm{E}+00$ & $0.00 \mathrm{E}+00$ & & $0.00 \mathrm{E}+00$ & $0.00 \mathrm{E}+00$ & \\
\hline $\mathrm{N}-13$ & $2.03 \mathrm{E}-08$ & $3.60 \mathrm{E}-08$ & & $1.53 \mathrm{E}-08$ & $3.20 \mathrm{E}-08$ & \\
\hline $\mathrm{O}-15$ & $1.96 \mathrm{E}-14$ & $2.20 \mathrm{E}-15$ & $\checkmark$ & $1.45 \mathrm{E}-14$ & $1.90 \mathrm{E}-15$ & $\checkmark$ \\
\hline F-18 & $2.23 \mathrm{E}-06$ & 4.80E-06 & & $1.61 \mathrm{E}-06$ & 4.00E-06 & \\
\hline $\mathrm{Na}-22$ & $3.83 \mathrm{E}-02$ & $1.00 \mathrm{E}-01$ & & $2.66 \mathrm{E}-02$ & $7.70 \mathrm{E}-02$ & \\
\hline $\mathrm{Na}-24$ & $6.42 \mathrm{E}-05$ & $1.50 \mathrm{E}-04$ & & $4.37 \mathrm{E}-05$ & $1.20 \mathrm{E}-04$ & \\
\hline $\mathrm{P}-32$ & $1.73 \mathrm{E}-04$ & $2.30 \mathrm{E}-03$ & & $1.56 \mathrm{E}-04$ & $1.70 \mathrm{E}-03$ & \\
\hline S-35 & $2.77 \mathrm{E}-05$ & $3.20 \mathrm{E}-04$ & & $2.66 \mathrm{E}-05$ & $2.50 \mathrm{E}-04$ & \\
\hline Ar-41 & 2.94E-06 & 6.30E-06 & & $1.63 \mathrm{E}-06$ & $4.10 \mathrm{E}-06$ & \\
\hline $\mathrm{K}-40$ & $2.49 \mathrm{E}-02$ & $9.60 \mathrm{E}-02$ & & 1.79E-02 & $7.40 \mathrm{E}-02$ & \\
\hline $\mathrm{Ca}-41$ & $3.33 \mathrm{E}-06$ & 8.00E-06 & & $2.28 \mathrm{E}-06$ & $6.10 \mathrm{E}-06$ & \\
\hline Sc-46 & $3.52 \mathrm{E}-03$ & 9.40E-03 & & $2.42 \mathrm{E}-03$ & 7.30E-03 & \\
\hline $\mathrm{Cr}-51$ & $2.18 \mathrm{E}-05$ & $6.80 \mathrm{E}-05$ & & $1.50 \mathrm{E}-05$ & $5.30 \mathrm{E}-05$ & \\
\hline Mn-54 & $5.21 \mathrm{E}-03$ & $1.30 \mathrm{E}-02$ & & $3.57 \mathrm{E}-03$ & 9.90E-03 & \\
\hline Mn-56 & 6.89E-06 & $1.50 \mathrm{E}-05$ & & $4.86 \mathrm{E}-06$ & $1.20 \mathrm{E}-05$ & \\
\hline $\mathrm{Fe}-55$ & 2.19E-05 & $1.80 \mathrm{E}-04$ & & 1.72E-05 & $1.40 \mathrm{E}-04$ & \\
\hline Fe-59 & $1.12 \mathrm{E}-03$ & $3.70 \mathrm{E}-03$ & & 7.76E-04 & $2.80 \mathrm{E}-03$ & \\
\hline Co-57 & $8.25 \mathrm{E}-04$ & $2.20 \mathrm{E}-03$ & & 5.69E-04 & $1.70 \mathrm{E}-03$ & \\
\hline Co-58 & $1.51 \mathrm{E}-03$ & $4.30 \mathrm{E}-03$ & & $1.04 \mathrm{E}-03$ & $3.30 \mathrm{E}-03$ & \\
\hline Co-60 & 7.82E-02 & $1.90 \mathrm{E}-01$ & & $5.36 \mathrm{E}-02$ & $1.50 \mathrm{E}-01$ & \\
\hline $\mathrm{Ni}-59$ & \begin{tabular}{|l|}
$7.60 \mathrm{E}-05$ \\
\end{tabular} & $2.40 \mathrm{E}-04$ & & $5.25 \mathrm{E}-05$ & \begin{tabular}{|l|}
$1.90 \mathrm{E}-04$ \\
\end{tabular} & \\
\hline $\mathrm{Ni}-63$ & $2.26 \mathrm{E}-05$ & $2.00 \mathrm{E}-04$ & & $1.68 \mathrm{E}-05$ & $1.60 \mathrm{E}-04$ & \\
\hline Ni-65 & $2.72 \mathrm{E}-06$ & 5.80E-06 & & 1.91E-06 & 4.70E-06 & \\
\hline $\mathrm{Cu}-64$ & $4.59 \mathrm{E}-06$ & $1.10 \mathrm{E}-05$ & & $3.08 \mathrm{E}-06$ & 8.10E-06 & \\
\hline $\mathrm{Zn}-65$ & $3.86 \mathrm{E}-03$ & 1.90E-02 & & $2.97 \mathrm{E}-03$ & $1.50 \mathrm{E}-02$ & \\
\hline Zn-69 & 8.37E-08 & 1.90E-07 & & $6.34 \mathrm{E}-08$ & $1.60 \mathrm{E}-07$ & \\
\hline $\mathrm{Zn}-69 \mathrm{~m}$ & $1.21 \mathrm{E}-05$ & 2.90E-05 & & $8.18 \mathrm{E}-06$ & 2.20E-05 & \\
\hline
\end{tabular}


Table E.1. (Contd)

\begin{tabular}{|c|c|c|c|c|c|c|}
\hline \multirow[b]{3}{*}{ Nuclide } & \multicolumn{3}{|c|}{ 10-m Release Height } & \multicolumn{3}{|c|}{ 40-m Release Height } \\
\hline & \multicolumn{3}{|c|}{$1.846 \mathrm{E}-07 \mathrm{~s} / \mathrm{m}^{3} \mathrm{CHI} / \mathrm{Q}$} & \multicolumn{3}{|c|}{$8.982 \mathrm{E}-08 \mathrm{~s} / \mathrm{m}^{3} \mathrm{CHI} / \mathrm{Q}$} \\
\hline & $\begin{array}{l}\text { Onsite } 2000 \mathrm{~h} / \mathrm{y}+ \\
\text { regional ingestion }\end{array}$ & $\begin{array}{c}\text { Offsite Resident } \\
\text { (mrem/y) }\end{array}$ & $\begin{array}{c}\text { Onsite }> \\
\text { Offsite }\end{array}$ & $\begin{array}{l}\text { Onsite } 2000 \mathrm{~h} / \mathrm{y}+ \\
\text { regional ingestion }\end{array}$ & \begin{tabular}{|c|}
$\begin{array}{c}\text { Offsite Resident } \\
(\mathrm{mrem} / \mathrm{y})\end{array}$ \\
\end{tabular} & Onsite $>$ Offsite \\
\hline Ga-67 & $1.51 \mathrm{E}-05$ & 4.70E-05 & & $1.02 \mathrm{E}-05$ & $3.60 \mathrm{E}-05$ & \\
\hline As-76 & $4.02 \mathrm{E}-05$ & $1.00 \mathrm{E}-04$ & & $2.67 \mathrm{E}-05$ & $7.60 \mathrm{E}-05$ & \\
\hline Br-82 & 9.32E-05 & $2.40 \mathrm{E}-04$ & & $6.41 \mathrm{E}-05$ & $1.90 \mathrm{E}-04$ & \\
\hline $\mathrm{Br}-83$ & 2.02E-08 & 4.40E-08 & & $1.44 \mathrm{E}-08$ & $3.60 \mathrm{E}-08$ & \\
\hline $\mathrm{Br}-84$ & 7.03E-07 & $1.70 \mathrm{E}-06$ & & $5.50 \mathrm{E}-07$ & $1.50 \mathrm{E}-06$ & \\
\hline $\mathrm{Br}-85$ & $1.98 \mathrm{E}-13$ & $6.00 \mathrm{E}-14$ & $\checkmark$ & $1.47 \mathrm{E}-13$ & $5.30 \mathrm{E}-14$ & $\checkmark$ \\
\hline $\mathrm{Kr}-83 \mathrm{~m}$ & 5.32E-10 & $1.10 \mathrm{E}-09$ & & 2.94E-10 & $7.40 \mathrm{E}-10$ & \\
\hline $\mathrm{Kr}-85$ & 2.24E-08 & $5.60 \mathrm{E}-08$ & & $1.09 \mathrm{E}-08$ & $3.00 \mathrm{E}-08$ & \\
\hline $\mathrm{Kr}-85 \mathrm{~m}$ & $6.55 \mathrm{E}-07$ & $1.50 \mathrm{E}-06$ & & $3.38 \mathrm{E}-07$ & $8.70 \mathrm{E}-07$ & \\
\hline $\mathrm{Kr}-87$ & $1.38 \mathrm{E}-06$ & $3.00 \mathrm{E}-06$ & & $7.96 \mathrm{E}-07$ & $2.00 \mathrm{E}-06$ & \\
\hline $\mathrm{Kr}-88$ & 7.16E-06 & $1.60 \mathrm{E}-05$ & & 3.79E-06 & 9.70E-06 & \\
\hline Kr-89 & $2.65 \mathrm{E}-11$ & 9.80E-12 & $\checkmark$ & $1.56 \mathrm{E}-11$ & 7.20E-12 & $\checkmark$ \\
\hline Kr-90 (b) & $0.00 \mathrm{E}+00$ & $0.00 \mathrm{E}+00$ & & $9.58 \mathrm{E}-38$ & $0.00 \mathrm{E}+00$ & $\checkmark$ \\
\hline $\mathrm{Rb}-86$ & $2.11 \mathrm{E}-04$ & $2.20 \mathrm{E}-03$ & & $1.78 \mathrm{E}-04$ & $1.70 \mathrm{E}-03$ & \\
\hline $\mathrm{Rb}-87$ & 5.83E-04 & 7.40E-03 & & $5.60 \mathrm{E}-04$ & $5.70 \mathrm{E}-03$ & \\
\hline $\mathrm{Rb}-88$ & 9.65E-08 & $2.20 \mathrm{E}-07$ & & $7.46 \mathrm{E}-08$ & $2.00 \mathrm{E}-07$ & \\
\hline Rb-89 & $1.78 \mathrm{E}-07$ & $4.00 \mathrm{E}-07$ & & $1.36 \mathrm{E}-07$ & $3.50 \mathrm{E}-07$ & \\
\hline Rb-90 & $2.26 \mathrm{E}-12$ & $5.40 \mathrm{E}-13$ & $\checkmark$ & $1.68 \mathrm{E}-12$ & $4.80 \mathrm{E}-13$ & $\checkmark$ \\
\hline Rb-90M & $6.41 \mathrm{E}-10$ & $4.50 \mathrm{E}-10$ & $\checkmark$ & $4.77 \mathrm{E}-10$ & $3.90 \mathrm{E}-10$ & $\checkmark$ \\
\hline Sr-89 & $8.38 \mathrm{E}-05$ & $1.30 \mathrm{E}-03$ & & $6.68 \mathrm{E}-05$ & $1.00 \mathrm{E}-03$ & \\
\hline Sr-90 & $5.81 \mathrm{E}-03$ & $8.80 \mathrm{E}-02$ & & 5.19E-03 & $6.80 \mathrm{E}-02$ & \\
\hline Sr-91 & $1.29 \mathrm{E}-05$ & $3.00 \mathrm{E}-05$ & & 8.74E-06 & $2.30 \mathrm{E}-05$ & \\
\hline Sr-92 & 6.32E-06 & $1.40 \mathrm{E}-05$ & & 4.43E-06 & $1.10 \mathrm{E}-05$ & \\
\hline $\mathrm{Y}-90$ & $6.55 \mathrm{E}-05$ & $2.60 \mathrm{E}-04$ & & 4.29E-05 & $2.00 \mathrm{E}-04$ & \\
\hline $\mathrm{Y}-90 \mathrm{~m}$ & 2.33E-06 & $5.10 \mathrm{E}-06$ & & $1.63 \mathrm{E}-06$ & 4.10E-06 & \\
\hline $\mathrm{Y}-91$ & $3.79 \mathrm{E}-04$ & $2.00 \mathrm{E}-03$ & & 2.54E-04 & $1.50 \mathrm{E}-03$ & \\
\hline $\mathrm{Y}-91 \mathrm{~m}$ & 4.29E-07 & 9.70E-07 & & 3.29E-07 & 8.60E-07 & \\
\hline Y-92 & 5.86E-06 & $1.30 \mathrm{E}-05$ & & 4.04E-06 & $1.00 \mathrm{E}-05$ & \\
\hline Y-93 & $1.73 \mathrm{E}-05$ & 4.00E-05 & & $1.15 \mathrm{E}-05$ & $3.00 \mathrm{E}-05$ & \\
\hline Zr-93 & $3.51 \mathrm{E}-04$ & 9.90E-04 & & $2.31 \mathrm{E}-04$ & 7.40E-04 & \\
\hline Zr-95 & $1.08 \mathrm{E}-03$ & $3.00 \mathrm{E}-03$ & & 7.37E-04 & $2.30 \mathrm{E}-03$ & \\
\hline $\mathrm{Nb}-93 \mathrm{~m}$ & 3.79E-04 & $1.60 \mathrm{E}-03$ & & 2.81E-04 & $1.20 \mathrm{E}-03$ & \\
\hline $\mathrm{Nb}-94$ & $2.59 \mathrm{E}-01$ & 6.30E-01 & & $1.78 \mathrm{E}-01$ & $4.90 \mathrm{E}-01$ & \\
\hline $\mathrm{Nb}-95$ & 7.64E-04 & $3.70 \mathrm{E}-03$ & & $5.73 \mathrm{E}-04$ & $2.90 \mathrm{E}-03$ & \\
\hline $\mathrm{Nb}-95 \mathrm{M}$ & $3.36 \mathrm{E}-05$ & $2.30 \mathrm{E}-04$ & & $2.58 \mathrm{E}-05$ & $1.80 \mathrm{E}-04$ & \\
\hline Nb-97 & $1.06 \mathrm{E}-06$ & $2.30 \mathrm{E}-06$ & & 7.89E-07 & 2.00E-06 & \\
\hline $\mathrm{Nb}-97 \mathrm{M}$ & $2.30 \mathrm{E}-22$ & $6.90 \mathrm{E}-25$ & $\checkmark$ & $1.71 \mathrm{E}-22$ & $6.00 \mathrm{E}-25$ & $\checkmark$ \\
\hline Mo-93 & 9.22E-04 & 2.20E-03 & & $6.30 \mathrm{E}-04$ & $1.70 \mathrm{E}-03$ & \\
\hline Mo-99 +D (c) & 4.94E-05 & $1.30 \mathrm{E}-04$ & & $3.32 \mathrm{E}-05$ & $1.00 \mathrm{E}-04$ & \\
\hline Tc-97 & $1.21 \mathrm{E}-03$ & $4.50 \mathrm{E}-03$ & & $8.83 \mathrm{E}-04$ & $3.50 \mathrm{E}-03$ & \\
\hline Tc-99 & $1.62 \mathrm{E}-03$ & $1.80 \mathrm{E}-02$ & & $1.58 \mathrm{E}-03$ & $1.40 \mathrm{E}-02$ & \\
\hline Tc-99m & $0.00 \mathrm{E}+00$ & $2.40 \mathrm{E}-06$ & & $0.00 \mathrm{E}+00$ & $1.90 \mathrm{E}-06$ & \\
\hline Tc-101 & $2.06 \mathrm{E}-08$ & $4.50 \mathrm{E}-08$ & & $1.58 \mathrm{E}-08$ & $4.00 \mathrm{E}-08$ & \\
\hline Ru-97 & $1.82 \mathrm{E}-05$ & $5.10 \mathrm{E}-05$ & & $1.24 \mathrm{E}-05$ & $3.90 \mathrm{E}-05$ & \\
\hline $\mathrm{Ru}-103$ & $4.68 \mathrm{E}-04$ & $1.40 \mathrm{E}-03$ & & $3.19 \mathrm{E}-04$ & $1.10 \mathrm{E}-03$ & \\
\hline
\end{tabular}


Table E.1. (Contd)

\begin{tabular}{|c|c|c|c|c|c|c|}
\hline \multirow[b]{3}{*}{ Nuclide } & \multicolumn{3}{|c|}{ 10-m Release Height } & \multicolumn{3}{|c|}{ 40-m Release Height } \\
\hline & \multicolumn{3}{|c|}{$1.846 \mathrm{E}-07 \mathrm{~s} / \mathrm{m}^{3} \mathrm{CHI} / \mathrm{Q}$} & \multicolumn{3}{|c|}{ 8.982E-08 s $/ \mathrm{m}^{3} \mathrm{CHI} / \mathrm{Q}$} \\
\hline & $\begin{array}{l}\text { Onsite } 2000 \mathrm{~h} / \mathrm{y}+ \\
\text { regional ingestion }\end{array}$ & $\begin{array}{c}\text { Offsite Resident } \\
(\mathrm{mrem} / \mathrm{y})\end{array}$ & $\begin{array}{c}\text { Onsite }> \\
\text { Offsite }\end{array}$ & $\begin{array}{l}\text { Onsite } 2000 \mathrm{~h} / \mathrm{y}+ \\
\text { regional ingestion }\end{array}$ & $\begin{array}{c}\text { Offsite Resident } \\
(\mathrm{mrem} / \mathrm{y})\end{array}$ & Onsite $>$ Offsite \\
\hline $\mathrm{Ru}-105$ & $6.84 \mathrm{E}-06$ & $1.50 \mathrm{E}-05$ & & $4.70 \mathrm{E}-06$ & $1.20 \mathrm{E}-05$ & \\
\hline Ru-106 & $3.53 \mathrm{E}-03$ & $1.20 \mathrm{E}-02$ & & $2.34 \mathrm{E}-03$ & $9.10 \mathrm{E}-03$ & \\
\hline Rh-103m & $0.00 \mathrm{E}+00$ & $2.40 \mathrm{E}-08$ & & $0.00 \mathrm{E}+00$ & $2.10 \mathrm{E}-08$ & \\
\hline Rh-105 & $1.09 \mathrm{E}-05$ & $3.60 \mathrm{E}-05$ & & $7.53 \mathrm{E}-06$ & $2.80 \mathrm{E}-05$ & \\
\hline Rh-105m & $6.98 \mathrm{E}-29$ & $7.70 \mathrm{E}-34$ & $\checkmark$ & $5.18 \mathrm{E}-29$ & $6.80 \mathrm{E}-34$ & $\checkmark$ \\
\hline Rh-106 (b) & $0.00 \mathrm{E}+00$ & $0.00 \mathrm{E}+00$ & & $0.00 \mathrm{E}+00$ & $0.00 \mathrm{E}+00$ & \\
\hline Pd-107 & $9.71 \mathrm{E}-05$ & $2.90 \mathrm{E}-04$ & & $6.49 \mathrm{E}-05$ & $2.20 \mathrm{E}-04$ & \\
\hline Pd-109 & $1.01 \mathrm{E}-05$ & $2.50 \mathrm{E}-05$ & & 6.72E-06 & $1.90 \mathrm{E}-05$ & \\
\hline Ag-109m & $1.41 \mathrm{E}-33$ & $1.10 \mathrm{E}-37$ & $\checkmark$ & $1.05 \mathrm{E}-33$ & $9.60 \mathrm{E}-38$ & $\checkmark$ \\
\hline Ag-110 (b) & $0.00 \mathrm{E}+00$ & $0.00 \mathrm{E}+00$ & & $0.00 \mathrm{E}+00$ & $0.00 \mathrm{E}+00$ & \\
\hline Ag-110m & $1.42 \mathrm{E}-02$ & $3.80 \mathrm{E}-02$ & & 9.80E-03 & $2.90 \mathrm{E}-02$ & \\
\hline Ag-111 & $1.01 \mathrm{E}-04$ & 7.80E-04 & & $8.29 \mathrm{E}-05$ & $6.00 \mathrm{E}-04$ & \\
\hline Cd-115 & 4.27E-05 & $1.50 \mathrm{E}-04$ & & $2.86 \mathrm{E}-05$ & $1.10 \mathrm{E}-04$ & \\
\hline Cd-115m & $3.85 \mathrm{E}-04$ & $2.80 \mathrm{E}-03$ & & $2.69 \mathrm{E}-04$ & $2.10 \mathrm{E}-03$ & \\
\hline In-113m & 5.91E-07 & $1.30 \mathrm{E}-06$ & & 4.29E-07 & $1.10 \mathrm{E}-06$ & \\
\hline In-115 & $8.56 \mathrm{E}-03$ & $4.70 \mathrm{E}-02$ & & $5.99 \mathrm{E}-03$ & $3.60 \mathrm{E}-02$ & \\
\hline In-115m & $1.59 \mathrm{E}-06$ & $3.50 \mathrm{E}-06$ & & $1.09 \mathrm{E}-06$ & $2.70 \mathrm{E}-06$ & \\
\hline Sn-113 & $2.23 \mathrm{E}-04$ & $1.70 \mathrm{E}-03$ & & $1.83 \mathrm{E}-04$ & $1.30 \mathrm{E}-03$ & \\
\hline Sn-123 & $1.68 \mathrm{E}-05$ & $4.00 \mathrm{E}-05$ & & $1.15 \mathrm{E}-05$ & $3.10 \mathrm{E}-05$ & \\
\hline Sn-125 & $2.05 \mathrm{E}-04$ & $1.80 \mathrm{E}-03$ & & $1.47 \mathrm{E}-04$ & $1.40 \mathrm{E}-03$ & \\
\hline Sn-126 & $1.14 \mathrm{E}-02$ & $3.70 \mathrm{E}-02$ & & $8.06 \mathrm{E}-03$ & $2.80 \mathrm{E}-02$ & \\
\hline Sb-124 & $2.33 \mathrm{E}-03$ & $6.70 \mathrm{E}-03$ & & $1.60 \mathrm{E}-03$ & $5.20 \mathrm{E}-03$ & \\
\hline Sb-125 & $8.38 \mathrm{E}-03$ & $2.10 \mathrm{E}-02$ & & $5.74 \mathrm{E}-03$ & $1.60 \mathrm{E}-02$ & \\
\hline Sb-126 & 8.07E-04 & $2.60 \mathrm{E}-03$ & & $5.52 \mathrm{E}-04$ & $2.00 \mathrm{E}-03$ & \\
\hline $\mathrm{Sb}-126 \mathrm{~m}$ & $2.12 \mathrm{E}-07$ & $5.00 \mathrm{E}-07$ & & $1.64 \mathrm{E}-07$ & $4.40 \mathrm{E}-07$ & \\
\hline Sb-127 & 9.96E-05 & $3.60 \mathrm{E}-04$ & & $6.70 \mathrm{E}-05$ & $2.70 \mathrm{E}-04$ & \\
\hline Te-125m & $1.01 \mathrm{E}-04$ & $8.10 \mathrm{E}-04$ & & 7.49E-05 & $6.20 \mathrm{E}-04$ & \\
\hline Te-127 & 2.49E-06 & $5.70 \mathrm{E}-06$ & & $1.65 \mathrm{E}-06$ & $4.30 \mathrm{E}-06$ & \\
\hline $\mathrm{Te}-127 \mathrm{~m}$ & 2.39E-04 & $2.00 \mathrm{E}-03$ & & $1.80 \mathrm{E}-04$ & $1.50 \mathrm{E}-03$ & \\
\hline Te-129 & 2.81E-07 & $6.10 \mathrm{E}-07$ & & 2.09E-07 & $5.30 \mathrm{E}-07$ & \\
\hline Te-129m & $2.45 \mathrm{E}-04$ & $2.00 \mathrm{E}-03$ & & $1.77 \mathrm{E}-04$ & $1.50 \mathrm{E}-03$ & \\
\hline Te-131 & $1.40 \mathrm{E}-07$ & $3.40 \mathrm{E}-07$ & & $1.10 \mathrm{E}-07$ & $3.00 \mathrm{E}-07$ & \\
\hline $\mathrm{Te}-131 \mathrm{~m}$ & 7.03E-05 & $1.80 \mathrm{E}-04$ & & $4.73 \mathrm{E}-05$ & $1.30 \mathrm{E}-04$ & \\
\hline Te-132 & 7.35E-05 & $3.20 \mathrm{E}-04$ & & 4.89E-05 & $2.40 \mathrm{E}-04$ & \\
\hline Te-133 & $3.90 \mathrm{E}-08$ & $8.00 \mathrm{E}-08$ & & $2.97 \mathrm{E}-08$ & $7.10 \mathrm{E}-08$ & \\
\hline Te-133m & $1.94 \mathrm{E}-06$ & $4.40 \mathrm{E}-06$ & & $1.48 \mathrm{E}-06$ & $3.80 \mathrm{E}-06$ & \\
\hline Te-134 & $4.88 \mathrm{E}-07$ & $1.10 \mathrm{E}-06$ & & 3.79E-07 & $1.00 \mathrm{E}-06$ & \\
\hline $\mathrm{I}-122$ & 1.19E-12 & $1.50 \mathrm{E}-12$ & & $3.58 \mathrm{E}-12$ & $2.50 \mathrm{E}-12$ & $\checkmark$ \\
\hline $\mathrm{I}-123$ & 5.51E-07 & $2.40 \mathrm{E}-06$ & & $2.27 \mathrm{E}-06$ & $5.50 \mathrm{E}-06$ & \\
\hline $\mathrm{I}-125$ & $2.14 \mathrm{E}-04$ & $4.50 \mathrm{E}-03$ & & $3.63 \mathrm{E}-04$ & $1.10 \mathrm{E}-02$ & \\
\hline I-129 & $4.65 \mathrm{E}-03$ & $7.60 \mathrm{E}-02$ & & $1.01 \mathrm{E}-02$ & $1.80 \mathrm{E}-01$ & \\
\hline I-130 & $6.13 \mathrm{E}-06$ & $2.70 \mathrm{E}-05$ & & $2.52 \mathrm{E}-05$ & $6.00 \mathrm{E}-05$ & \\
\hline I-131 & $1.21 \mathrm{E}-04$ & $2.50 \mathrm{E}-03$ & & $2.50 \mathrm{E}-04$ & $5.80 \mathrm{E}-03$ & \\
\hline I-132 & $7.85 \mathrm{E}-07$ & $3.50 \mathrm{E}-06$ & & $2.83 \mathrm{E}-06$ & $6.80 \mathrm{E}-06$ & \\
\hline I-133 & 4.17E-06 & $2.80 \mathrm{E}-05$ & & $1.58 \mathrm{E}-05$ & $6.40 \mathrm{E}-05$ & \\
\hline
\end{tabular}


Table E.1. (Contd)

\begin{tabular}{|c|c|c|c|c|c|c|}
\hline \multirow[b]{3}{*}{ Nuclide } & \multicolumn{3}{|c|}{ 10-m Release Height } & \multicolumn{3}{|c|}{ 40-m Release Height } \\
\hline & \multicolumn{3}{|c|}{$1.846 \mathrm{E}-07 \mathrm{~s} / \mathrm{m}^{3} \mathrm{CHI} / \mathrm{Q}$} & \multicolumn{3}{|c|}{$8.982 \mathrm{E}-08 \mathrm{~s} / \mathrm{m}^{3} \mathrm{CHI} / \mathrm{Q}$} \\
\hline & $\begin{array}{l}\text { Onsite } 2000 \mathrm{~h} / \mathrm{y}+ \\
\text { regional ingestion }\end{array}$ & $\begin{array}{c}\text { Offsite Resident } \\
(\mathrm{mrem} / \mathrm{y})\end{array}$ & $\begin{array}{c}\text { Onsite }> \\
\text { Offsite }\end{array}$ & $\begin{array}{l}\text { Onsite } 2000 \mathrm{~h} / \mathrm{y}+ \\
\text { regional ingestion }\end{array}$ & $\begin{array}{c}\text { Offsite Resident } \\
(\mathrm{mrem} / \mathrm{y})\end{array}$ & Onsite $>$ Offsite \\
\hline I-134 & $1.93 \mathrm{E}-07$ & $9.60 \mathrm{E}-07$ & & $5.91 \mathrm{E}-07$ & \begin{tabular}{|l|l}
$1.70 \mathrm{E}-06$ \\
\end{tabular} & \\
\hline $\mathrm{I}-135$ & $2.02 \mathrm{E}-06$ & 8.50E-06 & & $8.12 \mathrm{E}-06$ & $1.90 \mathrm{E}-05$ & \\
\hline $\mathrm{Xe}-122$ & $4.02 \mathrm{E}-07$ & $9.90 \mathrm{E}-07$ & & $1.98 \mathrm{E}-07$ & $5.40 \mathrm{E}-07$ & \\
\hline $\mathrm{Xe}-123$ & $1.62 \mathrm{E}-06$ & $3.50 \mathrm{E}-06$ & & $8.81 \mathrm{E}-07$ & $2.20 \mathrm{E}-06$ & \\
\hline $\mathrm{Xe}-125$ & $1.44 \mathrm{E}-06$ & $3.50 \mathrm{E}-06$ & & 7.14E-07 & $1.90 \mathrm{E}-06$ & \\
\hline $\mathrm{Xe}-127$ & $1.74 \mathrm{E}-06$ & $4.40 \mathrm{E}-06$ & & $8.46 \mathrm{E}-07$ & $2.30 \mathrm{E}-06$ & \\
\hline $\mathrm{Xe}-131 \mathrm{~m}$ & 6.34E-08 & $1.60 \mathrm{E}-07$ & & $3.08 \mathrm{E}-08$ & $8.50 \mathrm{E}-08$ & \\
\hline $\mathrm{Xe}-133$ & $2.30 \mathrm{E}-07$ & $5.70 \mathrm{E}-07$ & & $1.12 \mathrm{E}-07$ & $3.10 \mathrm{E}-07$ & \\
\hline $\mathrm{Xe}-133 \mathrm{~m}$ & $1.95 \mathrm{E}-07$ & $4.90 \mathrm{E}-07$ & & 9.54E-08 & $2.60 \mathrm{E}-07$ & \\
\hline Xe-135 & $1.29 \mathrm{E}-06$ & $3.10 \mathrm{E}-06$ & & $6.43 \mathrm{E}-07$ & $1.70 \mathrm{E}-06$ & \\
\hline $\mathrm{Xe}-135 \mathrm{~m}$ & $4.40 \mathrm{E}-08$ & 9.50E-08 & & 2.72E-08 & 7.00E-08 & \\
\hline $\mathrm{Xe}-137$ & $1.78 \mathrm{E}-11$ & 9.80E-12 & $\checkmark$ & $1.05 \mathrm{E}-11$ & $7.20 \mathrm{E}-12$ & $\checkmark$ \\
\hline $\mathrm{Xe}-138$ & $1.03 \mathrm{E}-07$ & $2.20 \mathrm{E}-07$ & & 6.32E-08 & $1.60 \mathrm{E}-07$ & \\
\hline Cs-134 & $2.45 \mathrm{E}-02$ & $7.80 \mathrm{E}-02$ & & $1.73 \mathrm{E}-02$ & $6.00 \mathrm{E}-02$ & \\
\hline Cs-134m & 2.42E-07 & 5.20E-07 & & $1.68 \mathrm{E}-07$ & 4.20E-07 & \\
\hline Cs-135 & 2.79E-04 & $3.50 \mathrm{E}-03$ & & 2.64E-04 & $2.70 \mathrm{E}-03$ & \\
\hline Cs-136 & $6.96 \mathrm{E}-04$ & $2.90 \mathrm{E}-03$ & & $4.94 \mathrm{E}-04$ & $2.20 \mathrm{E}-03$ & \\
\hline Cs-137 & 1.69E-03 & $2.10 \mathrm{E}-02$ & (d) & $6.36 \mathrm{E}-04$ & $1.60 \mathrm{E}-02$ & (d) \\
\hline Cs-137+D & 7.13E-02 & & (d) & $4.93 \mathrm{E}-02$ & & (d) \\
\hline Cs-138 & 9.70E-07 & 2.30E-06 & & 7.60E-07 & $2.10 \mathrm{E}-06$ & \\
\hline Cs-139 & 5.13E-09 & 8.80E-09 & & $3.86 \mathrm{E}-09$ & 7.80E-09 & \\
\hline Ba-133 & $2.42 \mathrm{E}-02$ & $5.90 \mathrm{E}-02$ & & $1.66 \mathrm{E}-02$ & $4.50 \mathrm{E}-02$ & \\
\hline $\mathrm{Ba}-133 \mathrm{~m}$ & $6.76 \mathrm{E}-06$ & $2.40 \mathrm{E}-05$ & & $4.53 \mathrm{E}-06$ & $1.80 \mathrm{E}-05$ & \\
\hline Ba-137m & $3.81 \mathrm{E}-13$ & $8.60 \mathrm{E}-14$ & $\checkmark$ & $2.83 \mathrm{E}-13$ & $7.50 \mathrm{E}-14$ & $\checkmark$ \\
\hline Ba-139 & 4.29E-07 & 9.20E-07 & & $3.13 \mathrm{E}-07$ & $7.80 \mathrm{E}-07$ & \\
\hline $\mathrm{Ba}-140+\mathrm{D}(\mathrm{c})$ & 6.39E-04 & $8.30 \mathrm{E}-04$ & & $4.38 \mathrm{E}-04$ & $6.40 \mathrm{E}-04$ & \\
\hline Ba-141 & $1.04 \mathrm{E}-07$ & $2.40 \mathrm{E}-07$ & & $8.10 \mathrm{E}-08$ & $2.20 \mathrm{E}-07$ & \\
\hline Ba-142 & $2.33 \mathrm{E}-08$ & 4.40E-08 & & $1.75 \mathrm{E}-08$ & $3.90 \mathrm{E}-08$ & \\
\hline La-140 & $1.15 \mathrm{E}-04$ & $3.00 \mathrm{E}-04$ & & $7.73 \mathrm{E}-05$ & $2.30 \mathrm{E}-04$ & \\
\hline La-141 & $1.83 \mathrm{E}-07$ & 4.00E-07 & & $1.27 \mathrm{E}-07$ & $3.20 \mathrm{E}-07$ & \\
\hline La-142 & 5.07E-06 & $1.10 \mathrm{E}-05$ & & $3.70 \mathrm{E}-06$ & $9.20 \mathrm{E}-06$ & \\
\hline Ce-141 & $1.27 \mathrm{E}-04$ & 5.90E-04 & & $8.65 \mathrm{E}-05$ & $4.50 \mathrm{E}-04$ & \\
\hline Ce-143 & $3.33 \mathrm{E}-05$ & 8.80E-05 & & $2.21 \mathrm{E}-05$ & $6.70 \mathrm{E}-05$ & \\
\hline Ce-144 & $2.87 \mathrm{E}-03$ & 9.60E-03 & & $1.90 \mathrm{E}-03$ & $7.20 \mathrm{E}-03$ & \\
\hline Pp-143 & 6.49E-05 & $4.60 \mathrm{E}-04$ & & $4.37 \mathrm{E}-05$ & $3.50 \mathrm{E}-04$ & \\
\hline $\mathrm{Pp}-144$ & $1.68 \mathrm{E}-08$ & $3.90 \mathrm{E}-08$ & & $1.30 \mathrm{E}-08$ & $3.40 \mathrm{E}-08$ & \\
\hline Pp-144m & $3.08 \mathrm{E}-10$ & 4.20E-10 & & $2.30 \mathrm{E}-10$ & $3.70 \mathrm{E}-10$ & \\
\hline Nd-147 & $8.55 \mathrm{E}-05$ & $4.50 \mathrm{E}-04$ & & $5.76 \mathrm{E}-05$ & $3.40 \mathrm{E}-04$ & \\
\hline Pm-147 & 2.84E-04 & $8.40 \mathrm{E}-04$ & & $1.87 \mathrm{E}-04$ & $6.20 \mathrm{E}-04$ & \\
\hline $\mathrm{Pm}-148$ & 1.44E-04 & 6.60E-04 & & 9.65E-05 & $5.00 \mathrm{E}-04$ & \\
\hline Pm-148m & $2.08 \mathrm{E}-03$ & $6.10 \mathrm{E}-03$ & & $1.42 \mathrm{E}-03$ & 4.70E-03 & \\
\hline Pm-149 & 2.33E-05 & 8.20E-05 & & $1.53 \mathrm{E}-05$ & $6.20 \mathrm{E}-05$ & \\
\hline Pm-151 & 9.10E-06 & $2.10 \mathrm{E}-05$ & & $6.23 \mathrm{E}-06$ & $1.70 \mathrm{E}-05$ & \\
\hline Sm-147 & 5.37E-01 & $1.30 \mathrm{E}+00$ & & $3.50 \mathrm{E}-01$ & 9.60E-01 & \\
\hline Sm-151 & 2.18E-04 & $5.80 \mathrm{E}-04$ & & $1.43 \mathrm{E}-04$ & $4.30 \mathrm{E}-04$ & \\
\hline
\end{tabular}


Table E.1. (Contd)

\begin{tabular}{|c|c|c|c|c|c|c|}
\hline \multirow[b]{3}{*}{ Nuclide } & \multicolumn{3}{|c|}{ 10-m Release Height } & \multicolumn{3}{|c|}{ 40-m Release Height } \\
\hline & \multicolumn{3}{|c|}{$1.846 \mathrm{E}-07 \mathrm{~s} / \mathrm{m}^{3} \mathrm{CHI} / \mathrm{Q}$} & \multicolumn{3}{|c|}{$8.982 \mathrm{E}-08 \mathrm{~s} / \mathrm{m}^{3} \mathrm{CHI} / \mathrm{Q}$} \\
\hline & $\begin{array}{l}\text { Onsite } 2000 \mathrm{~h} / \mathbf{y}+ \\
\text { regional ingestion }\end{array}$ & $\begin{array}{c}\text { Offsite Resident } \\
(\mathrm{mrem} / \mathrm{y})\end{array}$ & $\begin{array}{c}\text { Onsite }> \\
\text { Offsite }\end{array}$ & $\begin{array}{l}\text { Onsite } 2000 \mathrm{~h} / \mathrm{y}+ \\
\text { regional ingestion }\end{array}$ & $\begin{array}{c}\text { Offsite Resident } \\
(\mathrm{mrem} / \mathrm{y})\end{array}$ & Onsite $>$ Offsite \\
\hline Sm-153 & \begin{tabular}{|l|l}
$1.80 \mathrm{E}-05$ \\
\end{tabular} & $5.80 \mathrm{E}-05$ & & $1.19 \mathrm{E}-05$ & \begin{tabular}{|l|l|}
$4.40 \mathrm{E}-05$ \\
\end{tabular} & \\
\hline Eu-152 & $7.85 \mathrm{E}-02$ & $1.90 \mathrm{E}-01$ & & $5.36 \mathrm{E}-02$ & $1.50 \mathrm{E}-01$ & \\
\hline $\mathrm{Eu}-152 \mathrm{~m}$ & $3.01 \mathrm{E}-06$ & $6.90 \mathrm{E}-06$ & & $2.06 \mathrm{E}-06$ & $5.40 \mathrm{E}-06$ & \\
\hline Eu-154 & 6.33E-02 & $1.50 \mathrm{E}-01$ & & 4.34E-02 & $1.20 \mathrm{E}-01$ & \\
\hline Eu-155 & $2.50 \mathrm{E}-03$ & 6.30E-03 & & $1.70 \mathrm{E}-03$ & $4.80 \mathrm{E}-03$ & \\
\hline Eu-156 & $5.02 \mathrm{E}-04$ & $1.90 \mathrm{E}-03$ & & $3.44 \mathrm{E}-04$ & $1.50 \mathrm{E}-03$ & \\
\hline Tb-160 & $1.72 \mathrm{E}-03$ & $5.00 \mathrm{E}-03$ & & $1.18 \mathrm{E}-03$ & $3.90 \mathrm{E}-03$ & \\
\hline Но-166 & $2.51 \mathrm{E}-05$ & 6.60E-05 & & $1.65 \mathrm{E}-05$ & $4.90 \mathrm{E}-05$ & \\
\hline Ho- $166 \mathrm{~m}$ & $2.58 \mathrm{E}-01$ & 6.20E-01 & & $1.77 \mathrm{E}-01$ & $4.80 \mathrm{E}-01$ & \\
\hline Hf-181 & $5.87 \mathrm{E}-04$ & $1.90 \mathrm{E}-03$ & & $4.02 \mathrm{E}-04$ & $1.50 \mathrm{E}-03$ & \\
\hline W-181 & $1.15 \mathrm{E}-04$ & $3.60 \mathrm{E}-04$ & & 8.04E-05 & $2.70 \mathrm{E}-04$ & \\
\hline $\mathrm{W}-185$ & $3.24 \mathrm{E}-05$ & 4.70E-04 & & $3.02 \mathrm{E}-05$ & $3.60 \mathrm{E}-04$ & \\
\hline W-187 & $1.51 \mathrm{E}-05$ & $3.80 \mathrm{E}-05$ & & $1.02 \mathrm{E}-05$ & $2.90 \mathrm{E}-05$ & \\
\hline $\operatorname{Re}-187$ & $1.10 \mathrm{E}-06$ & $1.40 \mathrm{E}-05$ & & $9.53 \mathrm{E}-07$ & $1.00 \mathrm{E}-05$ & \\
\hline Ir-192 & $1.50 \mathrm{E}-03$ & 4.30E-03 & & $1.02 \mathrm{E}-03$ & $3.30 \mathrm{E}-03$ & \\
\hline $\mathrm{Hg}-203$ & $4.18 \mathrm{E}-04$ & $2.70 \mathrm{E}-03$ & & $3.28 \mathrm{E}-04$ & $2.10 \mathrm{E}-03$ & \\
\hline T1-207 & $6.41 \mathrm{E}-12$ & $5.30 \mathrm{E}-12$ & $\checkmark$ & $4.77 \mathrm{E}-12$ & $4.60 \mathrm{E}-12$ & $\checkmark$ \\
\hline T1-208 & $2.37 \mathrm{E}-11$ & $8.50 \mathrm{E}-12$ & $\checkmark$ & $1.77 \mathrm{E}-11$ & $7.40 \mathrm{E}-12$ & $\checkmark$ \\
\hline Tl-209 & $1.51 \mathrm{E}-13$ & $2.20 \mathrm{E}-14$ & $\checkmark$ & $1.13 \mathrm{E}-13$ & $1.90 \mathrm{E}-14$ & $\checkmark$ \\
\hline Pb-209 & $3.88 \mathrm{E}-07$ & $8.40 \mathrm{E}-07$ & & $2.67 \mathrm{E}-07$ & 6.60E-07 & \\
\hline $\mathrm{Pb}-210+\mathrm{D}(\mathrm{c})$ & $1.20 \mathrm{E}-01$ & $1.10 \mathrm{E}+00$ & & $8.55 \mathrm{E}-02$ & $8.40 \mathrm{E}-01$ & \\
\hline $\mathrm{Pb}-211$ & 6.43E-06 & $1.50 \mathrm{E}-05$ & & 5.02E-06 & $1.30 \mathrm{E}-05$ & \\
\hline $\mathrm{Pb}-212$ & 9.95E-04 & $2.30 \mathrm{E}-03$ & & $6.59 \mathrm{E}-04$ & $1.70 \mathrm{E}-03$ & \\
\hline $\mathrm{Pb}-214$ & 4.49E-07 & $1.10 \mathrm{E}-06$ & & $3.51 \mathrm{E}-07$ & $9.60 \mathrm{E}-07$ & \\
\hline Bi-210 & $1.39 \mathrm{E}-03$ & $3.40 \mathrm{E}-03$ & & 9.03E-04 & $2.50 \mathrm{E}-03$ & \\
\hline Bi-211 & $7.76 \mathrm{E}-14$ & $1.00 \mathrm{E}-14$ & $\checkmark$ & $5.77 \mathrm{E}-14$ & $8.80 \mathrm{E}-15$ & $\checkmark$ \\
\hline $\mathrm{Bi}-212$ & $4.65 \mathrm{E}-05$ & $1.00 \mathrm{E}-04$ & & $3.51 \mathrm{E}-05$ & 9.00E-05 & \\
\hline Bi-213 & $1.27 \mathrm{E}-06$ & $2.90 \mathrm{E}-06$ & & 9.77E-07 & $2.60 \mathrm{E}-06$ & \\
\hline $\mathrm{Bi}-214$ & $3.15 \mathrm{E}-07$ & $7.50 \mathrm{E}-07$ & & $2.46 \mathrm{E}-07$ & 6.60E-07 & \\
\hline Po-210 & 6.84E-02 & $4.10 \mathrm{E}-01$ & & $4.66 \mathrm{E}-02$ & $3.10 \mathrm{E}-01$ & \\
\hline At-217 (b) & $0.00 \mathrm{E}+00$ & $0.00 \mathrm{E}+00$ & & $0.00 \mathrm{E}+00$ & $0.00 \mathrm{E}+00$ & \\
\hline Rn-219 (e) & $2.17 \mathrm{E}-04$ & $(\mathrm{~Pb}-211)$ & & $1.54 \mathrm{E}-04$ & $(\mathrm{~Pb}-211)$ & \\
\hline Rn-220 (e) & $1.39 \mathrm{E}-06$ & $(\mathrm{~Pb}-212)$ & & 9.23E-07 & $(\mathrm{Pb}-212)$ & \\
\hline Rn-222 & $2.78 \mathrm{E}-05$ & 7.00E-05 & & $1.36 \mathrm{E}-05$ & $3.70 \mathrm{E}-05$ & \\
\hline Fr-221 & $3.08 \mathrm{E}-09$ & $2.60 \mathrm{E}-09$ & $\checkmark$ & $2.28 \mathrm{E}-09$ & $2.30 \mathrm{E}-09$ & \\
\hline Fr-223 & $7.55 \mathrm{E}-07$ & $1.80 \mathrm{E}-06$ & & 5.86E-07 & $1.60 \mathrm{E}-06$ & \\
\hline Ra-223 & $6.02 \mathrm{E}-02$ & $1.80 \mathrm{E}-01$ & & $3.95 \mathrm{E}-02$ & $1.40 \mathrm{E}-01$ & \\
\hline Ra-224 & 2.49E-02 & 6.50E-02 & & $1.62 \mathrm{E}-02$ & 4.90E-02 & \\
\hline Ra-225 & $3.09 \mathrm{E}-02$ & $1.20 \mathrm{E}-01$ & & $2.03 \mathrm{E}-02$ & $8.70 \mathrm{E}-02$ & \\
\hline Ra-226 & 7.00E-02 & $3.60 \mathrm{E}-01$ & & 4.73E-02 & $2.70 \mathrm{E}-01$ & \\
\hline Ra-228 & $2.02 \mathrm{E}-02$ & $1.50 \mathrm{E}-01$ & & $1.40 \mathrm{E}-02$ & $1.10 \mathrm{E}-01$ & \\
\hline Ac-225 & 4.39E-02 & $1.10 \mathrm{E}-01$ & & $2.86 \mathrm{E}-02$ & $8.00 \mathrm{E}-02$ & \\
\hline Ac-227 & $4.58 \mathrm{E}+00$ & $1.10 \mathrm{E}+01$ & & $3.00 \mathrm{E}+00$ & $8.40 \mathrm{E}+00$ & \\
\hline Ac-228 & $4.45 \mathrm{E}-04$ & $9.80 \mathrm{E}-04$ & & $2.99 \mathrm{E}-04$ & $7.50 \mathrm{E}-04$ & \\
\hline Th-227 & $8.42 \mathrm{E}-02$ & $2.00 \mathrm{E}-01$ & & $5.50 \mathrm{E}-02$ & $1.50 \mathrm{E}-01$ & \\
\hline
\end{tabular}


Table E.1. (Contd)

\begin{tabular}{|c|c|c|c|c|c|c|}
\hline \multirow[b]{3}{*}{ Nuclide } & \multicolumn{3}{|c|}{ 10-m Release Height } & \multicolumn{3}{|c|}{ 40-m Release Height } \\
\hline & \multicolumn{3}{|c|}{\begin{tabular}{|c|}
$1.846 \mathrm{E}-07 \mathrm{~s} / \mathrm{m}^{3} \mathrm{CHI} / \mathrm{Q}$ \\
\end{tabular}} & \multicolumn{3}{|c|}{ 8.982E-08 s $/ \mathrm{m}^{3} \mathrm{CHI} / \mathrm{Q}$} \\
\hline & $\begin{array}{l}\text { Onsite } 2000 \mathrm{~h} / \mathrm{y}+ \\
\text { regional ingestion }\end{array}$ & $\begin{array}{c}\text { Offsite Resident } \\
\text { (mrem/y) }\end{array}$ & $\begin{array}{c}\text { Onsite }> \\
\text { Offsite }\end{array}$ & $\begin{array}{l}\text { Onsite } 2000 \mathrm{~h} / \mathrm{y}+ \\
\text { regional ingestion }\end{array}$ & \begin{tabular}{|c|}
$\begin{array}{c}\text { Offsite Resident } \\
(\mathrm{mrem} / \mathrm{y})\end{array}$ \\
\end{tabular} & Onsite $>$ Offsite \\
\hline Th-228 & $1.82 \mathrm{E}+00$ & $4.30 \mathrm{E}+00$ & & $1.18 \mathrm{E}+00$ & $3.20 \mathrm{E}+00$ & \\
\hline Th-229 & $5.04 \mathrm{E}+00$ & $1.20 \mathrm{E}+01$ & & $3.29 \mathrm{E}+00$ & $8.90 \mathrm{E}+00$ & \\
\hline Th-230 & $1.79 \mathrm{E}+00$ & $4.30 \mathrm{E}+00$ & & $1.17 \mathrm{E}+00$ & $3.20 \mathrm{E}+00$ & \\
\hline Th-231 & $6.87 \mathrm{E}-06$ & $1.80 \mathrm{E}-05$ & & $4.52 \mathrm{E}-06$ & $1.30 \mathrm{E}-05$ & \\
\hline Th-232+D (c) & $2.99 \mathrm{E}+00$ & $6.20 \mathrm{E}+00$ & & $1.97 \mathrm{E}+00$ & $4.50 \mathrm{E}+00$ & \\
\hline Th-234 & $2.79 \mathrm{E}-04$ & $1.90 \mathrm{E}-03$ & & $1.89 \mathrm{E}-04$ & $1.40 \mathrm{E}-03$ & \\
\hline Pa-231 & $3.48 \mathrm{E}+00$ & $8.90 \mathrm{E}+00$ & & $2.27 \mathrm{E}+00$ & $6.60 \mathrm{E}+00$ & \\
\hline Pa-233 & $2.00 \mathrm{E}-04$ & $8.10 \mathrm{E}-04$ & & $1.37 \mathrm{E}-04$ & $6.20 \mathrm{E}-04$ & \\
\hline $\mathrm{Pa}-234$ & $1.98 \mathrm{E}-05$ & $4.50 \mathrm{E}-05$ & & $1.35 \mathrm{E}-05$ & $3.50 \mathrm{E}-05$ & \\
\hline Pa-234m & $9.26 \mathrm{E}-22$ & $7.80 \mathrm{E}-24$ & $\checkmark$ & $6.89 \mathrm{E}-22$ & $6.80 \mathrm{E}-24$ & $\checkmark$ \\
\hline U-232 & $3.46 \mathrm{E}+00$ & $8.60 \mathrm{E}+00$ & & $2.26 \mathrm{E}+00$ & $6.30 \mathrm{E}+00$ & \\
\hline U-233 & 9.66E-01 & $2.40 \mathrm{E}+00$ & & $6.30 \mathrm{E}-01$ & $1.80 \mathrm{E}+00$ & \\
\hline U-234 & 9.52E-01 & $2.40 \mathrm{E}+00$ & & 6.24E-01 & $1.80 \mathrm{E}+00$ & \\
\hline U-235 & 9.09E-01 & $2.30 \mathrm{E}+00$ & & $5.95 \mathrm{E}-01$ & $1.70 \mathrm{E}+00$ & \\
\hline U-236 & 9.04E-01 & $2.30 \mathrm{E}+00$ & & $5.91 \mathrm{E}-01$ & $1.70 \mathrm{E}+00$ & \\
\hline U-237 & 5.00E-05 & $2.40 \mathrm{E}-04$ & & $3.38 \mathrm{E}-05$ & $1.80 \mathrm{E}-04$ & \\
\hline U-238 & $8.48 \mathrm{E}-01$ & $2.10 \mathrm{E}+00$ & & 5.54E-01 & $1.60 \mathrm{E}+00$ & \\
\hline U-240 & $1.54 \mathrm{E}-05$ & $3.60 \mathrm{E}-05$ & & $1.02 \mathrm{E}-05$ & $2.70 \mathrm{E}-05$ & \\
\hline $\mathrm{Np}-237$ & $3.55 \mathrm{E}+00$ & $8.90 \mathrm{E}+00$ & & $2.32 \mathrm{E}+00$ & $6.60 \mathrm{E}+00$ & \\
\hline $\mathrm{Np}-238$ & $2.46 \mathrm{E}-04$ & $6.00 \mathrm{E}-04$ & & $1.61 \mathrm{E}-04$ & $4.50 \mathrm{E}-04$ & \\
\hline \begin{tabular}{|l|}
$\mathrm{Np}-239$ \\
\end{tabular} & 2.79E-05 & $9.10 \mathrm{E}-05$ & & $1.86 \mathrm{E}-05$ & $6.90 \mathrm{E}-05$ & \\
\hline $\mathrm{Np}-240$ & $1.44 \mathrm{E}-06$ & $3.20 \mathrm{E}-06$ & & $1.08 \mathrm{E}-06$ & 2.80E-06 & \\
\hline $\mathrm{Np}-240 \mathrm{~m}$ & 2.03E-09 & 2.90E-09 & & $1.51 \mathrm{E}-09$ & 2.50E-09 & \\
\hline $\mathrm{Pu}-236$ & 6.34E-01 & $1.60 \mathrm{E}+00$ & & 4.14E-01 & $1.20 \mathrm{E}+00$ & \\
\hline $\mathrm{Pu}-238$ & $2.29 \mathrm{E}+00$ & $5.90 \mathrm{E}+00$ & & $1.50 \mathrm{E}+00$ & $4.40 \mathrm{E}+00$ & \\
\hline $\mathrm{Pu}-239$ & $2.48 \mathrm{E}+00$ & $6.40 \mathrm{E}+00$ & & $1.61 \mathrm{E}+00$ & $4.70 \mathrm{E}+00$ & \\
\hline $\mathrm{Pu}-240$ & $2.47 \mathrm{E}+00$ & $6.40 \mathrm{E}+00$ & & $1.61 \mathrm{E}+00$ & $4.70 \mathrm{E}+00$ & \\
\hline $\mathrm{Pu}-241$ & $3.77 \mathrm{E}-02$ & $1.00 \mathrm{E}-01$ & & $2.47 \mathrm{E}-02$ & 7.40E-02 & \\
\hline $\mathrm{Pu}-242$ & $2.34 \mathrm{E}+00$ & $6.10 \mathrm{E}+00$ & & $1.53 \mathrm{E}+00$ & $4.50 \mathrm{E}+00$ & \\
\hline $\mathrm{Pu}-243$ & $1.22 \mathrm{E}-06$ & $2.70 \mathrm{E}-06$ & & $8.28 \mathrm{E}-07$ & $2.10 \mathrm{E}-06$ & \\
\hline $\mathrm{Pu}-244$ & $2.34 \mathrm{E}+00$ & $6.00 \mathrm{E}+00$ & & $1.52 \mathrm{E}+00$ & $4.50 \mathrm{E}+00$ & \\
\hline Am-241 & $3.91 \mathrm{E}+00$ & $9.80 \mathrm{E}+00$ & & $2.57 \mathrm{E}+00$ & $7.20 \mathrm{E}+00$ & \\
\hline Am-242 & $3.76 \mathrm{E}-04$ & $8.60 \mathrm{E}-04$ & & 2.49E-04 & $6.50 \mathrm{E}-04$ & \\
\hline Am-242m & $3.78 \mathrm{E}+00$ & $9.40 \mathrm{E}+00$ & & $2.45 \mathrm{E}+00$ & $7.00 \mathrm{E}+00$ & \\
\hline Am-243 & $3.91 \mathrm{E}+00$ & $9.80 \mathrm{E}+00$ & & $2.57 \mathrm{E}+00$ & $7.30 \mathrm{E}+00$ & \\
\hline $\mathrm{Cm}-242$ & $1.29 \mathrm{E}-01$ & $3.20 \mathrm{E}-01$ & & $8.42 \mathrm{E}-02$ & $2.40 \mathrm{E}-01$ & \\
\hline $\mathrm{Cm}-243$ & $2.63 \mathrm{E}+00$ & $6.60 \mathrm{E}+00$ & & $1.72 \mathrm{E}+00$ & $4.90 \mathrm{E}+00$ & \\
\hline $\mathrm{Cm}-244$ & $2.07 \mathrm{E}+00$ & $5.20 \mathrm{E}+00$ & & $1.35 \mathrm{E}+00$ & $3.80 \mathrm{E}+00$ & \\
\hline $\mathrm{Cm}-245$ & $4.05 \mathrm{E}+00$ & $1.00 \mathrm{E}+01$ & & $2.64 \mathrm{E}+00$ & $7.50 \mathrm{E}+00$ & \\
\hline $\mathrm{Cm}-246$ & $4.01 \mathrm{E}+00$ & $1.00 \mathrm{E}+01$ & & $2.61 \mathrm{E}+00$ & $7.40 \mathrm{E}+00$ & \\
\hline $\mathrm{Cm}-247$ & $3.73 \mathrm{E}+00$ & $9.30 \mathrm{E}+00$ & & $2.43 \mathrm{E}+00$ & $6.90 \mathrm{E}+00$ & \\
\hline $\mathrm{Cm}-248$ & $1.47 \mathrm{E}+01$ & $3.70 \mathrm{E}+01$ & & $9.61 \mathrm{E}+00$ & $2.70 \mathrm{E}+01$ & \\
\hline Cf-252 & $1.12 \mathrm{E}+00$ & $2.80 \mathrm{E}+00$ & & $7.28 \mathrm{E}-01$ & $2.00 \mathrm{E}+00$ & \\
\hline
\end{tabular}


Table E.1. (Contd)

(a) Onsite dose assumes exposure 2000h/y at the work location for inhalation and external pathways; ingestion dose is representative of an average member of the population.

(b) Very short-lived radionuclides result in no offsite dose.

(c) Includes dose from ingrowth of radioactive decay products in addition to the parent radionuclide.

(d) Offsite dose does not include ingrowth of Ba-137m decay product.

(e) Short-lived $\mathrm{Rn}$ isotopes were modeled based on the dose from their longer-lived progeny. For each Ci of Rn-219 released, $0.0018 \mathrm{Ci}$ of $\mathrm{Pb}-210$ is generated. Each $\mathrm{Ci}$ of $\mathrm{Rn}-220$ produces $0.0014 \mathrm{Ci}$ of $\mathrm{Pb}-212$. Dose is based on the $\mathrm{Pb}$ progeny times the appropriate equilibrium factor (Hill and Rittman 1999).

\section{E.1 Reference}

Hill, J. S., and P. D. Rittmann. 1999. Volume 1: Calculating Potential to Emit Releases and Doses for FEMPs and NOCs. HNF-3602, Vol. 1, Fluor Daniel Hanford, Inc., Richland, Washington. 


\section{Appendix F}

Comparison of Onsite Worker to Offsite Resident Doses for All Areas 


\section{Appendix F}

\section{Comparison of Onsite Worker to Offsite Resident Doses for All Areas}

Table F.1. Unit Dose Factors for 100 B/C Area for 10-m Releases - Total Dose to Individual, mrem/y per $\mathrm{Ci} / \mathrm{y}$ Release

\begin{tabular}{|c|c|c|c|c|c|}
\hline Nuclide & Offsite $M E I^{(a)}$ & $\begin{array}{c}\text { Onsite } \\
\text { Receptor }^{(b)} \text { Full- } \\
\text { time Exposure }\end{array}$ & $\begin{array}{c}\text { Onsite }> \\
\text { Offsite }\end{array}$ & $\begin{array}{c}\text { Onsite Receptor }^{(b)} \\
\text { Working-Year }^{(\text {oxposure }}{ }^{(d)}\end{array}$ & $\begin{array}{c}\text { Onsite }> \\
\text { Offsite }\end{array}$ \\
\hline $\mathrm{H}-3$ & $5.10 \mathrm{E}-05$ & $5.69 \mathrm{E}-06$ & & $4.43 \mathrm{E}-06$ & \\
\hline $\mathrm{C}-14$ & $4.00 \mathrm{E}-03$ & $2.41 \mathrm{E}-04$ & & $2.40 \mathrm{E}-04$ & \\
\hline $\mathrm{O}-15$ & $1.00 \mathrm{E}-09$ & $2.12 \mathrm{E}-17$ & & $1.81 \mathrm{E}-14$ & \\
\hline Ar-41 & $2.80 \mathrm{E}-05$ & $3.32 \mathrm{E}-06$ & & $9.65 \mathrm{E}-07$ & \\
\hline Mn-54 & $3.40 \mathrm{E}-02$ & $6.38 \mathrm{E}-03$ & & $1.89 \mathrm{E}-03$ & \\
\hline Co-60 & $5.10 \mathrm{E}-01$ & $9.54 \mathrm{E}-02$ & & $2.85 \mathrm{E}-02$ & \\
\hline $\mathrm{Zn}-65$ & $5.10 \mathrm{E}-02$ & $4.14 \mathrm{E}-03$ & & $1.75 \mathrm{E}-03$ & \\
\hline $\mathrm{Zn}-69 \mathrm{~m}$ & $8.70 \mathrm{E}-05$ & $1.36 \mathrm{E}-05$ & & $4.24 \mathrm{E}-06$ & \\
\hline $\mathrm{Kr}-85$ & $1.10 \mathrm{E}-07$ & $3.33 \mathrm{E}-08$ & & $1.45 \mathrm{E}-08$ & \\
\hline Sr-90 & $2.30 \mathrm{E}-01$ & $4.73 \mathrm{E}-03$ & & $3.53 \mathrm{E}-03$ & \\
\hline Zr-95 & $8.10 \mathrm{E}-03$ & $1.30 \mathrm{E}-03$ & & 3.99E-04 & \\
\hline $\mathrm{Nb}-95$ & $1.00 \mathrm{E}-02$ & $8.36 \mathrm{E}-04$ & & $3.33 \mathrm{E}-04$ & \\
\hline Ru-103 & $3.80 \mathrm{E}-03$ & 5.64E-04 & & $1.74 \mathrm{E}-04$ & \\
\hline $\mathrm{Ru}-106$ & $3.30 \mathrm{E}-02$ & $4.08 \mathrm{E}-03$ & & $1.50 \mathrm{E}-03$ & \\
\hline Ag-110 & $3.20 \mathrm{E}-25$ & $0.00 \mathrm{E}+00$ & & $1.52 \mathrm{E}-36$ & \\
\hline Sn-113 & $4.60 \mathrm{E}-03$ & $2.09 \mathrm{E}-04$ & & 1.19E-04 & \\
\hline Sb-125 & $5.50 \mathrm{E}-02$ & $1.02 \mathrm{E}-02$ & & 3.03E-03 & \\
\hline $\mathrm{I}-129$ & $2.70 \mathrm{E}-01$ & $3.80 \mathrm{E}-03$ & & $2.47 \mathrm{E}-03$ & \\
\hline $\mathrm{I}-131$ & $8.90 \mathrm{E}-03$ & $9.28 \mathrm{E}-05$ & & $6.42 \mathrm{E}-05$ & \\
\hline Cs-134 & $2.10 \mathrm{E}-01$ & $2.91 \mathrm{E}-02$ & & $9.43 \mathrm{E}-03$ & \\
\hline Cs-137+D & $4.96 \mathrm{E}-01$ & $8.64 \mathrm{E}-02$ & & $2.62 \mathrm{E}-02$ & \\
\hline Cs-137 & $5.50 \mathrm{E}-02$ & $1.26 \mathrm{E}-03$ & & $1.09 \mathrm{E}-03$ & \\
\hline $\mathrm{Ce}-144$ & $2.60 \mathrm{E}-02$ & $3.34 \mathrm{E}-03$ & & $1.21 \mathrm{E}-03$ & \\
\hline $\mathrm{Pm}-147$ & $2.30 \mathrm{E}-03$ & 3.32E-04 & & $1.21 \mathrm{E}-04$ & \\
\hline Eu-152 & $5.00 \mathrm{E}-01$ & $9.66 \mathrm{E}-02$ & & $2.87 \mathrm{E}-02$ & \\
\hline Eu-154 & $4.10 \mathrm{E}-01$ & 7.79E-02 & & $2.32 \mathrm{E}-02$ & \\
\hline Eu-155 & $1.70 \mathrm{E}-02$ & $3.06 \mathrm{E}-03$ & & $9.30 \mathrm{E}-04$ & \\
\hline Tl-208 & 5.90E-08 & $3.14 \mathrm{E}-13$ & & $3.12 \mathrm{E}-12$ & \\
\hline $\mathrm{Pb}-212$ & $7.20 \mathrm{E}-03$ & $1.09 \mathrm{E}-03$ & & $3.48 \mathrm{E}-04$ & \\
\hline Bi-212 & $6.90 \mathrm{E}-04$ & $4.53 \mathrm{E}-05$ & & $1.23 \mathrm{E}-05$ & \\
\hline U-234 & $6.50 \mathrm{E}+00$ & $1.11 \mathrm{E}+00$ & & 4.00E-01 & \\
\hline U-235 & $6.20 \mathrm{E}+00$ & $1.06 \mathrm{E}+00$ & & 3.80E-01 & \\
\hline U-238 & $5.80 \mathrm{E}+00$ & $9.91 \mathrm{E}-01$ & & $3.56 \mathrm{E}-01$ & \\
\hline $\mathrm{Pu}-239$ & $1.70 \mathrm{E}+01$ & $2.88 \mathrm{E}+00$ & & $1.04 \mathrm{E}+00$ & \\
\hline $\mathrm{Pu}-241$ & $2.70 \mathrm{E}-01$ & 4.39E-02 & & $1.58 \mathrm{E}-02$ & \\
\hline Am-241 & $2.70 \mathrm{E}+01$ & $4.57 \mathrm{E}+00$ & & $1.64 \mathrm{E}+00$ & \\
\hline
\end{tabular}


Table F.1. (Contd)

(a) Offsite receptor at $8600 \mathrm{~m} \mathrm{NNW}$ (HNF-3602, Hill and Rittmann 1999)

(b) Onsite receptor at LIGO, $26.3 \mathrm{~km} \mathrm{SE}$

(c) Assumes $8766 \mathrm{~h} / \mathrm{y}$ occupancy onsite for compliance with EPA regulations. Includes dose from ingestion of food produced regionally.

(d) Assumes $2000 \mathrm{~h} / \mathrm{y}$ occupancy onsite for compliance with DOE standards. Includes dose from ingestion of food produced regionally and exposure via inhalation and external pathways typical of an "average" member of the population for remainder of year $(6766 \mathrm{~h} / \mathrm{y})$. 
Table F.2. Unit Dose Factors for 100-D/DR Area for 100-m Releases - Total Dose to Individual, mrem/y per $\mathrm{Ci} / \mathrm{y}$ Release

\begin{tabular}{|c|c|c|c|c|c|}
\hline Nuclide & Offsite $M E I^{(a)}$ & $\begin{array}{c}\text { Onsite } \\
\text { Receptor }^{(b)} \text { Full- } \\
\text { time Exposure }^{(\mathrm{c})}\end{array}$ & $\begin{array}{c}\text { Onsite > } \\
\text { Offsite }\end{array}$ & $\begin{array}{c}\text { Onsite Receptor }^{(\mathrm{b})} \\
\text { Working-Year }^{\text {(d) }} \\
\text { Exposure }^{(\mathbf{s})}\end{array}$ & $\begin{array}{c}\text { Onsite > } \\
\text { Offsite }\end{array}$ \\
\hline $\mathrm{H}-3$ & $3.80 \mathrm{E}-05$ & $4.60 \mathrm{E}-06$ & & $4.18 \mathrm{E}-06$ & \\
\hline C-14 & $2.90 \mathrm{E}-03$ & $2.40 \mathrm{E}-04$ & & $2.40 \mathrm{E}-04$ & \\
\hline $\mathrm{O}-15$ & $4.70 \mathrm{E}-10$ & $1.07 \mathrm{E}-18$ & & $1.81 \mathrm{E}-14$ & \\
\hline Ar-41 & $1.70 \mathrm{E}-05$ & 8.24E-07 & & $3.96 \mathrm{E}-07$ & \\
\hline $\mathrm{Mn}-54$ & $2.40 \mathrm{E}-02$ & $2.66 \mathrm{E}-03$ & & $1.04 \mathrm{E}-03$ & \\
\hline Co-60 & $3.60 \mathrm{E}-01$ & 3.99E-02 & & $1.59 \mathrm{E}-02$ & \\
\hline $\mathrm{Zn}-65$ & $3.60 \mathrm{E}-02$ & $2.15 \mathrm{E}-03$ & & $1.30 \mathrm{E}-03$ & \\
\hline $\mathrm{Zn}-69 \mathrm{~m}$ & $5.80 \mathrm{E}-05$ & $5.05 \mathrm{E}-06$ & & $2.28 \mathrm{E}-06$ & \\
\hline $\mathrm{Kr}-85$ & $8.40 \mathrm{E}-08$ & $1.72 \mathrm{E}-08$ & & $1.09 \mathrm{E}-08$ & \\
\hline Sr-90 & $1.60 \mathrm{E}-01$ & $3.61 \mathrm{E}-03$ & & $3.27 \mathrm{E}-03$ & \\
\hline Zr-95 & $5.60 \mathrm{E}-03$ & $5.46 \mathrm{E}-04$ & & $2.27 \mathrm{E}-04$ & \\
\hline $\mathrm{Nb}-95$ & $6.90 \mathrm{E}-03$ & $4.16 \mathrm{E}-04$ & & $2.37 \mathrm{E}-04$ & \\
\hline $\mathrm{Ru}-103$ & $2.70 \mathrm{E}-03$ & $2.37 \mathrm{E}-04$ & & $9.98 \mathrm{E}-05$ & \\
\hline Ru-106 & $2.20 \mathrm{E}-02$ & $1.69 \mathrm{E}-03$ & & $9.59 \mathrm{E}-04$ & \\
\hline Ag-110 & $4.70 \mathrm{E}-26$ & $0.00 \mathrm{E}+00$ & & $1.52 \mathrm{E}-36$ & \\
\hline Sn-113 & $3.20 \mathrm{E}-03$ & $1.29 \mathrm{E}-04$ & & $1.01 \mathrm{E}-04$ & \\
\hline Sb-125 & $3.80 \mathrm{E}-02$ & $4.27 \mathrm{E}-03$ & & $1.68 \mathrm{E}-03$ & \\
\hline $\mathrm{I}-129$ & $1.50 \mathrm{E}-01$ & $2.73 \mathrm{E}-03$ & & $2.23 \mathrm{E}-03$ & \\
\hline $\mathrm{I}-131$ & $5.00 \mathrm{E}-03$ & $6.95 \mathrm{E}-05$ & & $5.88 \mathrm{E}-05$ & \\
\hline Cs-134 & $1.40 \mathrm{E}-01$ & $1.28 \mathrm{E}-02$ & & $5.71 \mathrm{E}-03$ & \\
\hline Cs-137+D & $3.42 \mathrm{E}-01$ & $3.65 \mathrm{E}-02$ & & $1.49 \mathrm{E}-02$ & \\
\hline Cs-137 & $3.80 \mathrm{E}-02$ & $1.10 \mathrm{E}-03$ & & $1.06 \mathrm{E}-03$ & \\
\hline $\mathrm{Ce}-144$ & $1.80 \mathrm{E}-02$ & $1.38 \mathrm{E}-03$ & & $7.66 \mathrm{E}-04$ & \\
\hline Pm-147 & $1.50 \mathrm{E}-03$ & $1.37 \mathrm{E}-04$ & & $7.69 \mathrm{E}-05$ & \\
\hline Eu-152 & $3.50 \mathrm{E}-01$ & $4.02 \mathrm{E}-02$ & & $1.58 \mathrm{E}-02$ & \\
\hline Eu-154 & $2.80 \mathrm{E}-01$ & $3.24 \mathrm{E}-02$ & & $1.28 \mathrm{E}-02$ & \\
\hline Eu-155 & $1.10 \mathrm{E}-02$ & $1.27 \mathrm{E}-03$ & & $5.21 \mathrm{E}-04$ & \\
\hline Tl-208 & $3.00 \mathrm{E}-08$ & $2.65 \mathrm{E}-14$ & & $3.05 \mathrm{E}-12$ & \\
\hline $\mathrm{Pb}-212$ & $4.70 \mathrm{E}-03$ & 3.84E-04 & & $1.87 \mathrm{E}-04$ & \\
\hline Bi-212 & $3.30 \mathrm{E}-04$ & $8.29 \mathrm{E}-06$ & & $3.82 \mathrm{E}-06$ & \\
\hline U-234 & $4.40 \mathrm{E}+00$ & $4.52 \mathrm{E}-01$ & & $2.49 \mathrm{E}-01$ & \\
\hline U-235 & $4.20 \mathrm{E}+00$ & $4.32 \mathrm{E}-01$ & & $2.36 \mathrm{E}-01$ & \\
\hline U-238 & $3.90 \mathrm{E}+00$ & 4.02E-01 & & $2.22 \mathrm{E}-01$ & \\
\hline $\mathrm{Pu}-239$ & $1.20 \mathrm{E}+01$ & $1.17 \mathrm{E}+00$ & & $6.45 \mathrm{E}-01$ & \\
\hline $\mathrm{Pu}-241$ & $1.90 \mathrm{E}-01$ & $1.79 \mathrm{E}-02$ & & $9.88 \mathrm{E}-03$ & \\
\hline Am-241 & $1.80 \mathrm{E}+01$ & $1.86 \mathrm{E}+00$ & & $1.02 \mathrm{E}+00$ & \\
\hline \multicolumn{6}{|c|}{$\begin{array}{l}\text { (a) Offsite receptor at } 8900 \mathrm{~m} \text { WNW (HNF-3602, Hill and Rittmann 1999) } \\
\text { (b) Onsite receptor at LIGO, } 27.9 \mathrm{~km} \text { SSE } \\
\text { (c) Assumes } 8766 \mathrm{~h} / \mathrm{y} \text { occupancy onsite for compliance with EPA regulations. Includes dose from } \\
\text { ingestion of food produced regionally. } \\
\text { (d) Assumes } 2000 \mathrm{~h} / \mathrm{y} \text { occupancy onsite for compliance with DOE standards. Includes dose from ingestion } \\
\text { of food produced regionally and exposure via inhalation and external pathways typical of an "average" } \\
\text { member of the population for remainder of year }(6766 \mathrm{~h} / \mathrm{y}) \text {. }\end{array}$} \\
\hline
\end{tabular}


Table F.3. Unit Dose Factors for 100-F Area for 10-m Releases - Total Dose to Individual, $\mathrm{mrem} / \mathrm{y}$ per $\mathrm{Ci} / \mathrm{y}$ Release

\begin{tabular}{|c|c|c|c|c|c|}
\hline Nuclide & ${\text { Offsite } M E I^{(a)}}$ & $\begin{array}{c}\text { Onsite } \\
\text { Receptor }^{(b)} \text { Full- } \\
\text { time Exposure }^{(\mathrm{c})}\end{array}$ & $\begin{array}{c}\text { Onsite > } \\
\text { Offsite }\end{array}$ & $\begin{array}{c}\text { Onsite Receptor }^{(\mathbf{b})} \\
\text { Working-Year }^{\text {Exposure }}{ }^{(\mathbf{d})}\end{array}$ & $\begin{array}{c}\text { Onsite > } \\
\text { Offsite }\end{array}$ \\
\hline H-3 & $6.50 \mathrm{E}-05$ & $5.22 \mathrm{E}-06$ & & $4.32 \mathrm{E}-06$ & \\
\hline C-14 & $5.10 \mathrm{E}-03$ & $2.40 \mathrm{E}-04$ & & $2.40 \mathrm{E}-04$ & \\
\hline $\mathrm{O}-15$ & $7.20 \mathrm{E}-10$ & $3.30 \mathrm{E}-16$ & & $1.82 \mathrm{E}-14$ & \\
\hline Ar-41 & $2.90 \mathrm{E}-05$ & $1.96 \mathrm{E}-06$ & & $6.55 \mathrm{E}-07$ & \\
\hline $\mathrm{Mn}-54$ & $3.80 \mathrm{E}-02$ & $5.22 \mathrm{E}-03$ & & $1.63 \mathrm{E}-03$ & \\
\hline Co-60 & $5.70 \mathrm{E}-01$ & 7.82E-02 & & $2.46 \mathrm{E}-02$ & \\
\hline $\mathrm{Zn}-65$ & $5.70 \mathrm{E}-02$ & $3.53 \mathrm{E}-03$ & & $1.61 \mathrm{E}-03$ & \\
\hline $\mathrm{Zn}-69 \mathrm{~m}$ & $9.40 \mathrm{E}-05$ & $1.09 \mathrm{E}-05$ & & $3.63 \mathrm{E}-06$ & \\
\hline $\mathrm{Kr}-85$ & $1.50 \mathrm{E}-07$ & $2.64 \mathrm{E}-08$ & & $1.30 \mathrm{E}-08$ & \\
\hline Sr-90 & $2.60 \mathrm{E}-01$ & $4.41 \mathrm{E}-03$ & & $3.45 \mathrm{E}-03$ & \\
\hline Zr-95 & $9.00 \mathrm{E}-03$ & $1.07 \mathrm{E}-03$ & & $3.47 \mathrm{E}-04$ & \\
\hline $\mathrm{Nb}-95$ & $1.10 \mathrm{E}-02$ & 7.06E-04 & & 3.03E-04 & \\
\hline Ru-103 & $4.30 \mathrm{E}-03$ & 4.63E-04 & & $1.51 \mathrm{E}-04$ & \\
\hline Ru-106 & $3.60 \mathrm{E}-02$ & $3.41 \mathrm{E}-03$ & & $1.35 \mathrm{E}-03$ & \\
\hline Ag-110 & $3.70 \mathrm{E}-27$ & $0.00 \mathrm{E}+00$ & & $1.52 \mathrm{E}-36$ & \\
\hline Sn-113 & $5.10 \mathrm{E}-03$ & $1.86 \mathrm{E}-04$ & & 1.14E-04 & \\
\hline Sb-125 & $6.10 \mathrm{E}-02$ & $8.39 \mathrm{E}-03$ & & $2.62 \mathrm{E}-03$ & \\
\hline $\mathrm{I}-129$ & $4.00 \mathrm{E}-01$ & $2.96 \mathrm{E}-03$ & & $2.28 \mathrm{E}-03$ & \\
\hline $\mathrm{I}-131$ & $1.30 \mathrm{E}-02$ & $7.46 \mathrm{E}-05$ & & $6.00 \mathrm{E}-05$ & \\
\hline Cs-134 & $2.30 \mathrm{E}-01$ & $2.40 \mathrm{E}-02$ & & $8.26 \mathrm{E}-03$ & \\
\hline Cs-137+D & $5.50 \mathrm{E}-01$ & $7.08 \mathrm{E}-02$ & & $2.27 \mathrm{E}-02$ & \\
\hline Cs-137 & $6.10 \mathrm{E}-02$ & $1.22 \mathrm{E}-03$ & & $1.08 \mathrm{E}-03$ & \\
\hline $\mathrm{Ce}-144$ & $2.90 \mathrm{E}-02$ & $2.79 \mathrm{E}-03$ & & $1.09 \mathrm{E}-03$ & \\
\hline Pm-147 & $2.50 \mathrm{E}-03$ & $2.77 \mathrm{E}-04$ & & $1.09 \mathrm{E}-04$ & \\
\hline Eu-152 & $5.60 \mathrm{E}-01$ & 7.91E-02 & & $2.47 \mathrm{E}-02$ & \\
\hline Eu-154 & $4.50 \mathrm{E}-01$ & $6.38 \mathrm{E}-02$ & & $2.00 \mathrm{E}-02$ & \\
\hline Eu-155 & $1.80 \mathrm{E}-02$ & $2.51 \mathrm{E}-03$ & & $8.04 \mathrm{E}-04$ & \\
\hline T1-208 & $5.90 \mathrm{E}-08$ & $1.45 \mathrm{E}-12$ & & $3.38 \mathrm{E}-12$ & \\
\hline $\mathrm{Pb}-212$ & $7.70 \mathrm{E}-03$ & 8.64E-04 & & $2.97 \mathrm{E}-04$ & \\
\hline Bi-212 & $6.00 \mathrm{E}-04$ & $2.15 \mathrm{E}-05$ & & $6.83 \mathrm{E}-06$ & \\
\hline U-234 & $7.20 \mathrm{E}+00$ & $9.27 \mathrm{E}-01$ & & $3.58 \mathrm{E}-01$ & \\
\hline U-235 & $6.90 \mathrm{E}+00$ & $8.85 \mathrm{E}-01$ & & $3.39 \mathrm{E}-01$ & \\
\hline $\mathrm{U}-238$ & $6.40 \mathrm{E}+00$ & $8.24 \mathrm{E}-01$ & & $3.18 \mathrm{E}-01$ & \\
\hline $\mathrm{Pu}-239$ & $1.90 \mathrm{E}+01$ & $2.40 \mathrm{E}+00$ & & $9.26 \mathrm{E}-01$ & \\
\hline $\mathrm{Pu}-241$ & $3.00 \mathrm{E}-01$ & $3.65 \mathrm{E}-02$ & & $1.41 \mathrm{E}-02$ & \\
\hline Am-241 & $2.90 \mathrm{E}+01$ & $3.81 \mathrm{E}+00$ & & $1.47 \mathrm{E}+00$ & \\
\hline \multicolumn{6}{|c|}{$\begin{array}{l}\text { (a) Offsite receptor at } 9700 \mathrm{~m} \text { ESE (HNF-3602, Hill and Rittmann 1999) } \\
\text { (b) Onsite receptor at Energy NW, } 22.4 \mathrm{~km} \text { SSE. } \\
\text { (c) Assumes } 8766 \mathrm{~h} / \mathrm{y} \text { occupancy onsite for compliance with EPA regulations. Includes dose from } \\
\text { ingestion of food produced regionally. } \\
\text { (d) Assumes } 2000 \mathrm{~h} / \mathrm{y} \text { occupancy onsite for compliance with DOE standards. Includes dose from ingestion } \\
\text { of food produced regionally and exposure via inhalation and external pathways typical of an "average" } \\
\text { member of the population for remainder of year }(6766 \mathrm{~h} / \mathrm{y}) \text {. }\end{array}$} \\
\hline
\end{tabular}


Table F.6. Unit Dose Factors for 100-N Area for 100-m Releases - Total Dose to Individual, mrem/y per Ci/y Release

\begin{tabular}{|c|c|c|c|c|c|}
\hline Nuclide & Offsite MEI $^{(a)}$ & $\begin{array}{c}\text { Onsite } \\
\text { Receptor }^{(b)} \text { Full- } \\
\text { time Exposure }^{(\mathrm{c})}\end{array}$ & $\begin{array}{c}\text { Onsite }> \\
\text { Offsite }\end{array}$ & $\begin{array}{c}\text { Onsite Receptor }^{(\mathrm{b})} \\
\text { Working-Year } \\
\text { Exposure }^{(\mathrm{d})}\end{array}$ & $\begin{array}{c}\text { Onsite }> \\
\text { Offsite }\end{array}$ \\
\hline $\mathrm{H}-3$ & $4.00 \mathrm{E}-05$ & $4.93 \mathrm{E}-06$ & & $4.25 \mathrm{E}-06$ & \\
\hline $\mathrm{C}-14$ & $3.10 \mathrm{E}-03$ & $2.40 \mathrm{E}-04$ & & $2.40 \mathrm{E}-04$ & \\
\hline $\mathrm{O}-15$ & $7.40 \mathrm{E}-10$ & $1.15 \mathrm{E}-18$ & & $1.81 \mathrm{E}-14$ & \\
\hline Ar-41 & $1.80 \mathrm{E}-05$ & $1.37 \mathrm{E}-06$ & & $5.20 \mathrm{E}-07$ & \\
\hline Mn-54 & $2.50 \mathrm{E}-02$ & $3.55 \mathrm{E}-03$ & & $1.25 \mathrm{E}-03$ & \\
\hline Co-60 & $3.80 \mathrm{E}-01$ & $5.32 \mathrm{E}-02$ & & $1.89 \mathrm{E}-02$ & \\
\hline Zn-65 & $3.80 \mathrm{E}-02$ & $2.63 \mathrm{E}-03$ & & $1.41 \mathrm{E}-03$ & \\
\hline $\mathrm{Zn}-69 \mathrm{~m}$ & $6.30 \mathrm{E}-05$ & $6.99 \mathrm{E}-06$ & & $2.73 \mathrm{E}-06$ & \\
\hline $\mathrm{Kr}-85$ & $8.90 \mathrm{E}-08$ & $2.21 \mathrm{E}-08$ & & $1.20 \mathrm{E}-08$ & \\
\hline Sr-90 & $1.70 \mathrm{E}-01$ & $3.88 \mathrm{E}-03$ & & $3.33 \mathrm{E}-03$ & \\
\hline Zr-95 & $6.00 \mathrm{E}-03$ & $7.27 \mathrm{E}-04$ & & $2.68 \mathrm{E}-04$ & \\
\hline $\mathrm{Nb}-95$ & $7.40 \mathrm{E}-03$ & $5.17 \mathrm{E}-04$ & & $2.60 \mathrm{E}-04$ & \\
\hline $\mathrm{Ru}-103$ & $2.90 \mathrm{E}-03$ & $3.15 \mathrm{E}-04$ & & $1.18 \mathrm{E}-04$ & \\
\hline Ru-106 & $2.40 \mathrm{E}-02$ & $2.25 \mathrm{E}-03$ & & $1.09 \mathrm{E}-03$ & \\
\hline Ag-110 & $3.30 \mathrm{E}-25$ & $0.00 \mathrm{E}+00$ & & $1.52 \mathrm{E}-36$ & \\
\hline Sn-113 & $3.40 \mathrm{E}-03$ & $1.48 \mathrm{E}-04$ & & $1.05 \mathrm{E}-04$ & \\
\hline Sb-125 & $4.10 \mathrm{E}-02$ & $5.71 \mathrm{E}-03$ & & $2.01 \mathrm{E}-03$ & \\
\hline $\mathrm{I}-129$ & $1.70 \mathrm{E}-01$ & $3.12 \mathrm{E}-03$ & & $2.32 \mathrm{E}-03$ & \\
\hline $\mathrm{I}-131$ & $5.60 \mathrm{E}-03$ & 7.79E-05 & & $6.08 \mathrm{E}-05$ & \\
\hline Cs-134 & $1.60 \mathrm{E}-01$ & $1.67 \mathrm{E}-02$ & & $6.60 \mathrm{E}-03$ & \\
\hline Cs-137+D & $3.69 \mathrm{E}-01$ & 4.84E-02 & & $1.76 \mathrm{E}-02$ & \\
\hline Cs-137 & $4.10 \mathrm{E}-02$ & $1.14 \mathrm{E}-03$ & & $1.06 \mathrm{E}-03$ & \\
\hline Ce-144 & $1.90 \mathrm{E}-02$ & $1.85 \mathrm{E}-03$ & & $8.74 \mathrm{E}-04$ & \\
\hline Pm-147 & $1.70 \mathrm{E}-03$ & $1.83 \mathrm{E}-04$ & & $8.74 \mathrm{E}-05$ & \\
\hline Eu-152 & $3.70 \mathrm{E}-01$ & $5.37 \mathrm{E}-02$ & & $1.89 \mathrm{E}-02$ & \\
\hline Eu-154 & $3.00 \mathrm{E}-01$ & $4.33 \mathrm{E}-02$ & & $1.53 \mathrm{E}-02$ & \\
\hline Eu-155 & $1.20 \mathrm{E}-02$ & $1.70 \mathrm{E}-03$ & & $6.19 \mathrm{E}-04$ & \\
\hline Tl-208 & $4.10 \mathrm{E}-08$ & $3.80 \mathrm{E}-14$ & & $3.05 \mathrm{E}-12$ & \\
\hline $\mathrm{Pb}-212$ & $5.10 \mathrm{E}-03$ & $5.40 \mathrm{E}-04$ & & $2.23 \mathrm{E}-04$ & \\
\hline Bi-212 & $3.70 \mathrm{E}-04$ & $1.86 \mathrm{E}-05$ & & $6.17 \mathrm{E}-06$ & \\
\hline U-234 & $4.80 \mathrm{E}+00$ & $6.07 \mathrm{E}-01$ & & $2.85 \mathrm{E}-01$ & \\
\hline U-235 & $4.60 \mathrm{E}+00$ & $5.81 \mathrm{E}-01$ & & $2.70 \mathrm{E}-01$ & \\
\hline U-238 & $4.20 \mathrm{E}+00$ & $5.41 \mathrm{E}-01$ & & $2.54 \mathrm{E}-01$ & \\
\hline $\mathrm{Pu}-239$ & $1.30 \mathrm{E}+01$ & $1.57 \mathrm{E}+00$ & & $7.36 \mathrm{E}-01$ & \\
\hline $\mathrm{Pu}-241$ & $2.00 \mathrm{E}-01$ & $2.40 \mathrm{E}-02$ & & $1.13 \mathrm{E}-02$ & \\
\hline Am-241 & $2.00 \mathrm{E}+01$ & $2.50 \mathrm{E}+00$ & & $1.17 \mathrm{E}+00$ & \\
\hline \multicolumn{6}{|c|}{$\begin{array}{l}\text { (a) Offsite receptor at } 8500 \mathrm{~m} \mathrm{WNW.} \\
\text { (b) Onsite receptor at Energy NW, } 28.8 \mathrm{~km} \text { SE. } \\
\text { (c) Assumes } 8766 \mathrm{~h} / \mathrm{y} \text { occupancy onsite for compliance with EPA regulations. Includes dose from } \\
\text { ingestion of food produced regionally. } \\
\text { (d) Assumes } 2000 \mathrm{~h} / \mathrm{y} \text { occupancy onsite for compliance with DOE standards. Includes dose from ingestion } \\
\text { of food produced regionally and exposure via inhalation and external pathways typical of an "average" } \\
\text { member of the population for remainder of year }(6766 \mathrm{~h} / \mathrm{y}) \text {. }\end{array}$} \\
\hline
\end{tabular}


Table F.7. Unit Dose Factors for 200-E Area for 10-m Releases - Total Dose to Individual, $\mathrm{mrem} / \mathrm{y}$ per $\mathrm{Ci} / \mathrm{y}$ Release

\begin{tabular}{|c|c|c|c|c|c|}
\hline Nuclide & Offsite $M E I^{(a)}$ & $\begin{array}{c}\text { Onsite } \\
\text { Receptor }^{(\mathbf{b})} \text { Full- }^{\text {(c) }} \\
\text { time Exposure }^{(\mathrm{c})}\end{array}$ & $\begin{array}{c}\text { Onsite }> \\
\text { Offsite }\end{array}$ & $\begin{array}{c}\text { Onsite Receptor }^{(b)} \\
\text { Working-Year }^{\left({ }^{(b)}\right.} \\
\text { Exposure }^{(\mathbf{y})}\end{array}$ & $\begin{array}{c}\text { Onsite }> \\
\text { Offsite }\end{array}$ \\
\hline $\mathrm{H}-3$ & $2.50 \mathrm{E}-05$ & $7.78 \mathrm{E}-06$ & & 4.67E-06 & \\
\hline C-14 & $1.90 \mathrm{E}-03$ & $2.24 \mathrm{E}-04$ & & $2.23 \mathrm{E}-04$ & \\
\hline $\mathrm{O}-15$ & $1.90 \mathrm{E}-14$ & $7.33 \mathrm{E}-13$ & $\checkmark$ & $1.67 \mathrm{E}-13$ & $\checkmark$ \\
\hline Ar-41 & $1.20 \mathrm{E}-05$ & $1.58 \mathrm{E}-05$ & $\checkmark$ & $4.06 \mathrm{E}-06$ & \\
\hline $\mathrm{Mn}-54$ & $1.60 \mathrm{E}-02$ & $2.04 \mathrm{E}-02$ & $\checkmark$ & $5.33 \mathrm{E}-03$ & \\
\hline Co-60 & $2.50 \mathrm{E}-01$ & $3.05 \mathrm{E}-01$ & $\checkmark$ & $8.01 \mathrm{E}-02$ & \\
\hline Zn-65 & $2.50 \mathrm{E}-02$ & $1.20 \mathrm{E}-02$ & & $3.99 \mathrm{E}-03$ & \\
\hline $\mathrm{Zn}-69 \mathrm{~m}$ & $4.10 \mathrm{E}-05$ & $5.13 \mathrm{E}-05$ & $\checkmark$ & $1.38 \mathrm{E}-05$ & \\
\hline $\mathrm{Kr}-85$ & $5.50 \mathrm{E}-08$ & $6.81 \mathrm{E}-08$ & $\checkmark$ & $2.20 \mathrm{E}-08$ & \\
\hline Sr-90 & $1.10 \mathrm{E}-01$ & $1.06 \mathrm{E}-02$ & & $6.20 \mathrm{E}-03$ & \\
\hline Zr-95 & $3.90 \mathrm{E}-03$ & $4.17 \mathrm{E}-03$ & $\checkmark$ & $1.11 \mathrm{E}-03$ & \\
\hline $\mathrm{Nb}-95$ & $4.80 \mathrm{E}-03$ & $2.49 \mathrm{E}-03$ & & $7.88 \mathrm{E}-04$ & \\
\hline Ru-103 & $1.80 \mathrm{E}-03$ & $1.81 \mathrm{E}-03$ & $\checkmark$ & $4.83 \mathrm{E}-04$ & \\
\hline Ru-106 & $1.60 \mathrm{E}-02$ & $1.34 \mathrm{E}-02$ & & $3.95 \mathrm{E}-03$ & \\
\hline Ag-110 & $0.00 \mathrm{E}+00$ & $0.00 \mathrm{E}+00$ & & $0.00 \mathrm{E}+00$ & \\
\hline Sn-113 & $2.20 \mathrm{E}-03$ & 5.62E-04 & & 2.39E-04 & \\
\hline Sb-125 & $2.60 \mathrm{E}-02$ & $3.29 \mathrm{E}-02$ & $\checkmark$ & $8.59 \mathrm{E}-03$ & \\
\hline $\mathrm{I}-129$ & $2.00 \mathrm{E}-01$ & $2.28 \mathrm{E}-02$ & & $8.77 \mathrm{E}-03$ & \\
\hline $\mathrm{I}-131$ & $6.40 \mathrm{E}-03$ & 5.23E-04 & & $2.15 \mathrm{E}-04$ & \\
\hline Cs-134 & $1.00 \mathrm{E}-01$ & $9.13 \mathrm{E}-02$ & & $2.51 \mathrm{E}-02$ & \\
\hline Cs-137+D & $2.46 \mathrm{E}-01$ & $2.75 \mathrm{E}-01$ & $\checkmark$ & $7.29 \mathrm{E}-02$ & \\
\hline Cs-137 & $2.70 \mathrm{E}-02$ & $2.40 \mathrm{E}-03$ & & $1.79 \mathrm{E}-03$ & \\
\hline $\mathrm{Ce}-144$ & $1.20 \mathrm{E}-02$ & $1.10 \mathrm{E}-02$ & & $3.21 \mathrm{E}-03$ & \\
\hline Pm-147 & $1.10 \mathrm{E}-03$ & $1.10 \mathrm{E}-03$ & & $3.21 \mathrm{E}-04$ & \\
\hline Eu-152 & $2.40 \mathrm{E}-01$ & $3.10 \mathrm{E}-01$ & $\checkmark$ & $8.10 \mathrm{E}-02$ & \\
\hline Eu-154 & $2.00 \mathrm{E}-01$ & $2.50 \mathrm{E}-01$ & $\checkmark$ & $6.54 \mathrm{E}-02$ & \\
\hline Eu-155 & $8.00 \mathrm{E}-03$ & $9.83 \mathrm{E}-03$ & $\checkmark$ & $2.60 \mathrm{E}-03$ & \\
\hline Tl-208 & $4.20 \mathrm{E}-11$ & $5.37 \mathrm{E}-10$ & $\checkmark$ & $1.23 \mathrm{E}-10$ & $\checkmark$ \\
\hline $\mathrm{Pb}-212$ & $3.30 \mathrm{E}-03$ & $4.31 \mathrm{E}-03$ & $\checkmark$ & $1.18 \mathrm{E}-03$ & \\
\hline Bi-212 & $2.70 \mathrm{E}-04$ & 4.04E-04 & $\checkmark$ & $9.73 \mathrm{E}-05$ & \\
\hline U-234 & $3.10 \mathrm{E}+00$ & $3.70 \mathrm{E}+00$ & $\checkmark$ & $1.07 \mathrm{E}+00$ & \\
\hline U-235 & $3.00 \mathrm{E}+00$ & $3.52 \mathrm{E}+00$ & $\checkmark$ & $1.02 \mathrm{E}+00$ & \\
\hline $\mathrm{U}-238$ & $2.80 \mathrm{E}+00$ & $3.28 \mathrm{E}+00$ & $\checkmark$ & $9.52 \mathrm{E}-01$ & \\
\hline $\mathrm{Pu}-239$ & $8.20 \mathrm{E}+00$ & $9.57 \mathrm{E}+00$ & $\checkmark$ & $2.77 \mathrm{E}+00$ & \\
\hline $\mathrm{Pu}-241$ & $1.30 \mathrm{E}-01$ & $1.45 \mathrm{E}-01$ & $\checkmark$ & $4.22 \mathrm{E}-02$ & \\
\hline $\mathrm{Am}-241$ & $1.30 \mathrm{E}+01$ & $1.52 \mathrm{E}+01$ & $\checkmark$ & $4.40 \mathrm{E}+00$ & \\
\hline \multicolumn{6}{|c|}{$\begin{array}{l}\text { (a) Offsite receptor at 20,200 m ESE (HNF-3602, Hill and Rittmann 1999) } \\
\text { (b) Onsite receptor at Energy NW, } 16.6 \mathrm{~km} \text { ESE. } \\
\text { (c) Assumes } 8766 \mathrm{~h} / \mathrm{y} \text { occupancy onsite for compliance with EPA regulations. Includes dose from } \\
\text { ingestion of food produced regionally. } \\
\text { (d) Assumes } 2000 \mathrm{~h} / \mathrm{y} \text { occupancy onsite for compliance with DOE standards. Includes dose from ingestion } \\
\text { of food produced regionally and exposure via inhalation and external pathways typical of an "average" } \\
\text { member of the population for remainder of year }(6766 \mathrm{~h} / \mathrm{y}) \text {. }\end{array}$} \\
\hline
\end{tabular}


Table F.9. Unit Dose Factors for 306 Bldg for 10-m Releases - Total Dose to Individual, $\mathrm{mrem} / \mathrm{y}$ per $\mathrm{Ci} / \mathrm{y}$ Release

\begin{tabular}{|c|c|c|c|c|c|}
\hline Nuclide & Offsite MEI ${ }^{(a)}$ & $\begin{array}{c}\text { Onsite } \\
\text { Receptor }^{(b)} \text { Full- } \\
\text { time Exposure }^{(c)}\end{array}$ & $\begin{array}{c}\text { Onsite > } \\
\text { Offsite }\end{array}$ & $\begin{array}{c}\text { Onsite Receptor }^{(\mathbf{b})} \\
\text { Working-Year }^{\text {Exposure }}{ }^{(\mathbf{d})}\end{array}$ & $\begin{array}{c}\text { Onsite }> \\
\text { Offsite }\end{array}$ \\
\hline $\mathrm{H}-3$ & $3.40 \mathrm{E}-04$ & $5.97 \mathrm{E}-04$ & $\checkmark$ & 1.42E-04 & \\
\hline $\mathrm{C}-14$ & $2.70 \mathrm{E}-02$ & 4.94E-04 & & $4.53 \mathrm{E}-04$ & \\
\hline O-15 & 4.30E-05 & 1.89E-03 & $\checkmark$ & 4.31E-04 & $\checkmark$ \\
\hline Ar-41 & $2.80 \mathrm{E}-04$ & $3.42 \mathrm{E}-03$ & $\checkmark$ & 7.82E-04 & $\checkmark$ \\
\hline $\mathrm{Mn}-54$ & $2.70 \mathrm{E}-01$ & $3.16 \mathrm{E}+00$ & $\checkmark$ & $7.23 \mathrm{E}-01$ & $\checkmark$ \\
\hline Co-60 & $4.10 \mathrm{E}+00$ & $4.72 \mathrm{E}+01$ & $\checkmark$ & $1.08 \mathrm{E}+01$ & $\checkmark$ \\
\hline Zn-65 & $4.10 \mathrm{E}-01$ & $1.69 \mathrm{E}+00$ & $\checkmark$ & $3.89 \mathrm{E}-01$ & \\
\hline $\mathrm{Zn}-69 \mathrm{~m}$ & $7.60 \mathrm{E}-04$ & $8.69 \mathrm{E}-03$ & $\checkmark$ & $1.99 \mathrm{E}-03$ & $\checkmark$ \\
\hline $\mathrm{Kr}-85$ & $7.70 \mathrm{E}-07$ & $8.76 \mathrm{E}-06$ & $\checkmark$ & $2.01 \mathrm{E}-06$ & $\checkmark$ \\
\hline Sr-90 & $1.90 \mathrm{E}+00$ & $9.93 \mathrm{E}-01$ & & $2.36 \mathrm{E}-01$ & \\
\hline Zr-95 & $6.50 \mathrm{E}-02$ & $6.44 \mathrm{E}-01$ & $\checkmark$ & $1.47 \mathrm{E}-01$ & $\checkmark$ \\
\hline $\mathrm{Nb}-95$ & $8.00 \mathrm{E}-02$ & $3.58 \mathrm{E}-01$ & $\checkmark$ & $8.23 \mathrm{E}-02$ & $\checkmark$ \\
\hline Ru-103 & $3.10 \mathrm{E}-02$ & $2.79 \mathrm{E}-01$ & $\checkmark$ & $6.38 \mathrm{E}-02$ & $\checkmark$ \\
\hline Ru-106 & $2.70 \mathrm{E}-01$ & $2.11 \mathrm{E}+00$ & $\checkmark$ & 4.84E-01 & $\checkmark$ \\
\hline Ag-110 & $8.50 \mathrm{E}-09$ & $2.05 \mathrm{E}-05$ & $\checkmark$ & $4.68 \mathrm{E}-06$ & $\checkmark$ \\
\hline Sn-113 & $3.70 \mathrm{E}-02$ & $7.08 \mathrm{E}-02$ & $\checkmark$ & $1.64 \mathrm{E}-02$ & \\
\hline Sb-125 & $4.40 \mathrm{E}-01$ & $5.08 \mathrm{E}+00$ & $\checkmark$ & $1.16 \mathrm{E}+00$ & $\checkmark$ \\
\hline I-129 & $3.60 \mathrm{E}+01$ & $3.83 \mathrm{E}+01$ & $\checkmark$ & $8.76 \mathrm{E}+00$ & \\
\hline $\mathrm{I}-131$ & $1.20 \mathrm{E}+00$ & $8.47 \mathrm{E}-01$ & & $1.94 \mathrm{E}-01$ & \\
\hline Cs-134 & $1.70 \mathrm{E}+00$ & $1.38 \mathrm{E}+01$ & $\checkmark$ & $3.16 \mathrm{E}+00$ & $\checkmark$ \\
\hline Cs-137+D & $3.97 \mathrm{E}+00$ & $4.23 \mathrm{E}+01$ & $\checkmark$ & $9.68 \mathrm{E}+00$ & $\checkmark$ \\
\hline Cs-137 & 4.40E-01 & $1.41 \mathrm{E}-01$ & & $3.50 \mathrm{E}-02$ & \\
\hline Ce-144 & $2.10 \mathrm{E}-01$ & $1.73 \mathrm{E}+00$ & $\checkmark$ & $3.96 \mathrm{E}-01$ & $\checkmark$ \\
\hline Pm-147 & $1.80 \mathrm{E}-02$ & $1.73 \mathrm{E}-01$ & $\checkmark$ & $3.96 \mathrm{E}-02$ & $\checkmark$ \\
\hline Eu-152 & $4.00 \mathrm{E}+00$ & $4.79 \mathrm{E}+01$ & $\checkmark$ & $1.10 \mathrm{E}+01$ & $\checkmark$ \\
\hline Eu-154 & $3.30 \mathrm{E}+00$ & $3.87 \mathrm{E}+01$ & $\checkmark$ & $8.85 \mathrm{E}+00$ & $\checkmark$ \\
\hline Eu-155 & $1.30 \mathrm{E}-01$ & $1.52 \mathrm{E}+00$ & $\checkmark$ & $3.48 \mathrm{E}-01$ & $\checkmark$ \\
\hline T1-208 & $2.60 \mathrm{E}-04$ & $8.16 \mathrm{E}-03$ & $\checkmark$ & $1.86 \mathrm{E}-03$ & $\checkmark$ \\
\hline $\mathrm{Pb}-212$ & $6.40 \mathrm{E}-02$ & $7.55 \mathrm{E}-01$ & $\checkmark$ & $1.73 \mathrm{E}-01$ & $\checkmark$ \\
\hline Bi-212 & $1.20 \mathrm{E}-02$ & $1.51 \mathrm{E}-01$ & $\checkmark$ & $3.45 \mathrm{E}-02$ & $\checkmark$ \\
\hline U-234 & $5.30 \mathrm{E}+01$ & $5.85 \mathrm{E}+02$ & $\checkmark$ & $1.34 \mathrm{E}+02$ & $\checkmark$ \\
\hline U-235 & $5.10 \mathrm{E}+01$ & $5.57 \mathrm{E}+02$ & $\checkmark$ & $1.28 \mathrm{E}+02$ & $\checkmark$ \\
\hline $\mathrm{U}-238$ & $4.70 \mathrm{E}+01$ & $5.20 \mathrm{E}+02$ & $\checkmark$ & $1.19 \mathrm{E}+02$ & $\checkmark$ \\
\hline $\mathrm{Pu}-239$ & $1.40 \mathrm{E}+02$ & $1.51 \mathrm{E}+03$ & $\checkmark$ & $3.46 \mathrm{E}+02$ & $\checkmark$ \\
\hline $\mathrm{Pu}-241$ & $2.20 \mathrm{E}+00$ & $2.30 \mathrm{E}+01$ & $\checkmark$ & $5.27 \mathrm{E}+00$ & $\checkmark$ \\
\hline Am-241 & $2.20 \mathrm{E}+02$ & $2.40 \mathrm{E}+03$ & $\checkmark$ & $5.50 \mathrm{E}+02$ & $\checkmark$ \\
\hline \multicolumn{6}{|c|}{$\begin{array}{l}\text { (a) Offsite receptor at } 1400 \mathrm{~m} \text { NE (HNF-3602, Hill and Rittmann 1999) } \\
\text { (b) Onsite receptor at } 313 \text { Building (Kaiser), } 140 \mathrm{~m} \mathrm{NW} \text {. } \\
\text { (c) Assumes } 8766 \mathrm{~h} / \mathrm{y} \text { occupancy onsite for compliance with EPA regulations. Includes dose from } \\
\text { ingestion of food produced regionally. } \\
\text { (d) Assumes } 2000 \mathrm{~h} / \mathrm{y} \text { occupancy onsite for compliance with DOE standards. Includes dose from ingestion } \\
\text { of food produced regionally and exposure via inhalation and external pathways typical of an "average" } \\
\text { member of the population for remainder of year }(6766 \mathrm{~h} / \mathrm{y}) .\end{array}$} \\
\hline
\end{tabular}


Table F.10. Unit Dose Factors for 324 Bldg for 10-m Releases - Total Dose to Individual, $\mathrm{mrem} / \mathrm{y}$ per $\mathrm{Ci} / \mathrm{y}$ Release

\begin{tabular}{|c|c|c|c|c|c|}
\hline Nuclide & ${\text { Offsite } M E I^{(a)}}^{(a)}$ & $\begin{array}{c}\text { Onsite } \\
\text { Receptor }^{(b)} \text { Full- } \\
\text { time Exposure }^{(c)}\end{array}$ & $\begin{array}{c}\text { Onsite > } \\
\text { Offsite }\end{array}$ & $\begin{array}{c}\text { Onsite Receptor }^{(b)} \\
\text { Working-Year }^{\left({ }^{(d)}\right.} \\
\text { Exposure }^{(2)}\end{array}$ & $\begin{array}{c}\text { Onsite }> \\
\text { Offsite }\end{array}$ \\
\hline $\mathrm{H}-3$ & $3.40 \mathrm{E}-04$ & $2.68 \mathrm{E}-04$ & & $6.69 \mathrm{E}-05$ & \\
\hline C-14 & $2.70 \mathrm{E}-02$ & $4.64 \mathrm{E}-04$ & & $4.46 \mathrm{E}-04$ & \\
\hline O-15 & 4.30E-05 & $3.38 \mathrm{E}-04$ & $\checkmark$ & $7.71 \mathrm{E}-05$ & $\checkmark$ \\
\hline Ar-41 & $2.80 \mathrm{E}-04$ & $1.49 \mathrm{E}-03$ & $\checkmark$ & $3.42 \mathrm{E}-04$ & $\checkmark$ \\
\hline Mn-54 & $2.70 \mathrm{E}-01$ & $1.36 \mathrm{E}+00$ & $\checkmark$ & $3.12 \mathrm{E}-01$ & $\checkmark$ \\
\hline Co-60 & $4.10 \mathrm{E}+00$ & $2.04 \mathrm{E}+01$ & $\checkmark$ & $4.68 \mathrm{E}+00$ & $\checkmark$ \\
\hline $\mathrm{Zn}-65$ & 4.10E-01 & 7.33E-01 & $\checkmark$ & $1.70 \mathrm{E}-01$ & \\
\hline $\mathrm{Zn}-69 \mathrm{~m}$ & $7.60 \mathrm{E}-04$ & $3.79 \mathrm{E}-03$ & $\checkmark$ & $8.71 \mathrm{E}-04$ & $\checkmark$ \\
\hline $\mathrm{Kr}-85$ & $7.70 \mathrm{E}-07$ & $3.89 \mathrm{E}-06$ & $\checkmark$ & $9.00 \mathrm{E}-07$ & $\checkmark$ \\
\hline Sr-90 & $1.90 \mathrm{E}+00$ & 4.42E-01 & & $1.10 \mathrm{E}-01$ & \\
\hline Zr-95 & $6.50 \mathrm{E}-02$ & $2.78 \mathrm{E}-01$ & $\checkmark$ & $6.38 \mathrm{E}-02$ & \\
\hline $\mathrm{Nb}-95$ & $8.00 \mathrm{E}-02$ & $1.55 \mathrm{E}-01$ & $\checkmark$ & $3.60 \mathrm{E}-02$ & \\
\hline Ru-103 & $3.10 \mathrm{E}-02$ & $1.21 \mathrm{E}-01$ & $\checkmark$ & $2.78 \mathrm{E}-02$ & \\
\hline Ru-106 & $2.70 \mathrm{E}-01$ & 9.29E-01 & $\checkmark$ & $2.14 \mathrm{E}-01$ & \\
\hline Ag-110 & $8.50 \mathrm{E}-09$ & 4.19E-07 & $\checkmark$ & $9.56 \mathrm{E}-08$ & $\checkmark$ \\
\hline Sn-113 & $3.70 \mathrm{E}-02$ & $3.11 \mathrm{E}-02$ & & $7.35 \mathrm{E}-03$ & \\
\hline Sb-125 & 4.40E-01 & $2.19 \mathrm{E}+00$ & $\checkmark$ & $5.02 \mathrm{E}-01$ & $\checkmark$ \\
\hline $\mathrm{I}-129$ & $3.60 \mathrm{E}+01$ & $1.39 \mathrm{E}+01$ & & $3.20 \mathrm{E}+00$ & \\
\hline $\mathrm{I}-131$ & $1.20 \mathrm{E}+00$ & $3.08 \mathrm{E}-01$ & & $7.07 \mathrm{E}-02$ & \\
\hline Cs-134 & $1.70 \mathrm{E}+00$ & $5.97 \mathrm{E}+00$ & $\checkmark$ & $1.37 \mathrm{E}+00$ & \\
\hline Cs-137+D & $3.97 \mathrm{E}+00$ & $1.83 \mathrm{E}+01$ & $\checkmark$ & $4.19 \mathrm{E}+00$ & $\checkmark$ \\
\hline Cs-137 & 4.40E-01 & $6.38 \mathrm{E}-02$ & & $1.75 \mathrm{E}-02$ & \\
\hline $\mathrm{Ce}-144$ & $2.10 \mathrm{E}-01$ & $7.60 \mathrm{E}-01$ & $\checkmark$ & $1.75 \mathrm{E}-01$ & \\
\hline Pm-147 & $1.80 \mathrm{E}-02$ & 7.59E-02 & $\checkmark$ & $1.75 \mathrm{E}-02$ & \\
\hline Eu-152 & $4.00 \mathrm{E}+00$ & $2.07 \mathrm{E}+01$ & $\checkmark$ & $4.75 \mathrm{E}+00$ & $\checkmark$ \\
\hline Eu-154 & $3.30 \mathrm{E}+00$ & $1.67 \mathrm{E}+01$ & $\checkmark$ & $3.83 \mathrm{E}+00$ & $\checkmark$ \\
\hline Eu-155 & $1.30 \mathrm{E}-01$ & $6.57 \mathrm{E}-01$ & $\checkmark$ & $1.51 \mathrm{E}-01$ & $\checkmark$ \\
\hline T1-208 & $2.60 \mathrm{E}-04$ & $1.89 \mathrm{E}-03$ & $\checkmark$ & $4.31 \mathrm{E}-04$ & $\checkmark$ \\
\hline $\mathrm{Pb}-212$ & $6.40 \mathrm{E}-02$ & $3.32 \mathrm{E}-01$ & $\checkmark$ & $7.63 \mathrm{E}-02$ & $\checkmark$ \\
\hline $\mathrm{Bi}-212$ & $1.20 \mathrm{E}-02$ & $6.40 \mathrm{E}-02$ & $\checkmark$ & $1.46 \mathrm{E}-02$ & $\checkmark$ \\
\hline U-234 & $5.30 \mathrm{E}+01$ & $2.57 \mathrm{E}+02$ & $\checkmark$ & $5.92 \mathrm{E}+01$ & $\checkmark$ \\
\hline U-235 & $5.10 \mathrm{E}+01$ & $2.45 \mathrm{E}+02$ & $\checkmark$ & $5.64 \mathrm{E}+01$ & $\checkmark$ \\
\hline $\mathrm{U}-238$ & $4.70 \mathrm{E}+01$ & $2.29 \mathrm{E}+02$ & $\checkmark$ & $5.28 \mathrm{E}+01$ & $\checkmark$ \\
\hline $\mathrm{Pu}-239$ & $1.40 \mathrm{E}+02$ & $6.64 \mathrm{E}+02$ & $\checkmark$ & $1.53 \mathrm{E}+02$ & $\checkmark$ \\
\hline $\mathrm{Pu}-241$ & $2.20 \mathrm{E}+00$ & $1.01 \mathrm{E}+01$ & $\checkmark$ & $2.33 \mathrm{E}+00$ & $\checkmark$ \\
\hline Am-241 & $2.20 \mathrm{E}+02$ & $1.06 \mathrm{E}+03$ & $\checkmark$ & $2.44 \mathrm{E}+02$ & $\checkmark$ \\
\hline \multicolumn{6}{|c|}{$\begin{array}{l}\text { (a) Offsite receptor at } 1400 \mathrm{~m} \text { NE (HNF-3602, Hill and Rittmann 1999) } \\
\text { (b) Onsite receptor at } 313 \text { Building (Kaiser), } 540 \mathrm{~m} \mathrm{NW} \text {. } \\
\text { (c) Assumes } 8766 \mathrm{~h} / \mathrm{y} \text { occupancy onsite for compliance with EPA regulations. Includes dose from } \\
\text { ingestion of food produced regionally. } \\
\text { (d) Assumes } 2000 \mathrm{~h} / \mathrm{y} \text { occupancy onsite for compliance with DOE standards. Includes dose from ingestion } \\
\text { of food produced regionally and exposure via inhalation and external pathways typical of an "average" } \\
\text { member of the population for remainder of year }(6766 \mathrm{~h} / \mathrm{y}) .\end{array}$} \\
\hline
\end{tabular}


Table F.11. Unit Dose Factors for 324 Bldg for 40-m Releases - Total Dose to Individual, mrem/y per $\mathrm{Ci} / \mathrm{y}$ Release

\begin{tabular}{|c|c|c|c|c|c|}
\hline Nuclide & ${\text { Offsite } M E I^{(a)}}^{(a)}$ & $\begin{array}{c}\text { Onsite } \\
\text { Receptor }^{(b)} \text { Full- } \\
\text { time Exposure }^{(c)}\end{array}$ & $\begin{array}{c}\text { Onsite > } \\
\text { Offsite }\end{array}$ & $\begin{array}{c}\text { Onsite Receptor }^{(b)} \\
\text { Working-Year }^{\text {Exposure }}{ }^{(\mathbf{d})}\end{array}$ & $\begin{array}{c}\text { Onsite }> \\
\text { Offsite }\end{array}$ \\
\hline $\mathrm{H}-3$ & $1.20 \mathrm{E}-04$ & $2.91 \mathrm{E}-05$ & & $9.82 \mathrm{E}-06$ & \\
\hline C-14 & $9.20 \mathrm{E}-03$ & $2.48 \mathrm{E}-04$ & & $2.46 \mathrm{E}-04$ & \\
\hline $\mathrm{O}-15$ & $1.80 \mathrm{E}-05$ & $3.64 \mathrm{E}-05$ & $\checkmark$ & $8.31 \mathrm{E}-06$ & \\
\hline Ar-41 & $9.80 \mathrm{E}-05$ & $1.45 \mathrm{E}-04$ & $\checkmark$ & $3.42 \mathrm{E}-05$ & \\
\hline Mn-54 & $1.00 \mathrm{E}-01$ & $1.68 \mathrm{E}-01$ & $\checkmark$ & $3.95 \mathrm{E}-02$ & \\
\hline Co-60 & $1.60 \mathrm{E}+00$ & $2.50 \mathrm{E}+00$ & $\checkmark$ & 5.89E-01 & \\
\hline $\mathrm{Zn}-65$ & $1.60 \mathrm{E}-01$ & $9.11 \mathrm{E}-02$ & & $2.30 \mathrm{E}-02$ & \\
\hline $\mathrm{Zn}-69 \mathrm{~m}$ & $2.70 \mathrm{E}-04$ & $4.07 \mathrm{E}-04$ & $\checkmark$ & $9.72 \mathrm{E}-05$ & \\
\hline $\mathrm{Kr}-85$ & $2.60 \mathrm{E}-07$ & $3.80 \mathrm{E}-07$ & $\checkmark$ & $9.38 \mathrm{E}-08$ & \\
\hline Sr-90 & $7.10 \mathrm{E}-01$ & 5.03E-02 & & $1.80 \mathrm{E}-02$ & \\
\hline Zr-95 & $2.50 \mathrm{E}-02$ & $3.36 \mathrm{E}-02$ & $\checkmark$ & $7.95 \mathrm{E}-03$ & \\
\hline $\mathrm{Nb}-95$ & $3.00 \mathrm{E}-02$ & $1.91 \mathrm{E}-02$ & & $4.75 \mathrm{E}-03$ & \\
\hline Ru-103 & $1.20 \mathrm{E}-02$ & $1.45 \mathrm{E}-02$ & $\checkmark$ & $3.44 \mathrm{E}-03$ & \\
\hline Ru-106 & $9.60 \mathrm{E}-02$ & $9.18 \mathrm{E}-02$ & & $2.25 \mathrm{E}-02$ & \\
\hline Ag-110 & $3.90 \mathrm{E}-09$ & $5.12 \mathrm{E}-08$ & $\checkmark$ & $1.17 \mathrm{E}-08$ & $\checkmark$ \\
\hline Sn-113 & $1.40 \mathrm{E}-02$ & $3.46 \mathrm{E}-03$ & & $9.81 \mathrm{E}-04$ & \\
\hline Sb-125 & $1.60 \mathrm{E}-01$ & $2.70 \mathrm{E}-01$ & $\checkmark$ & $6.35 \mathrm{E}-02$ & \\
\hline $\mathrm{I}-129$ & $2.00 \mathrm{E}+01$ & $1.74 \mathrm{E}+00$ & & $4.41 \mathrm{E}-01$ & \\
\hline $\mathrm{I}-131$ & $6.50 \mathrm{E}-01$ & $3.87 \mathrm{E}-02$ & & $1.00 \mathrm{E}-02$ & \\
\hline Cs-134 & $6.30 \mathrm{E}-01$ & $7.38 \mathrm{E}-01$ & $\checkmark$ & $1.76 \mathrm{E}-01$ & \\
\hline Cs-137+D & $1.53 \mathrm{E}+00$ & $2.26 \mathrm{E}+00$ & $\checkmark$ & 5.33E-01 & \\
\hline Cs-137 & $1.70 \mathrm{E}-01$ & $8.62 \mathrm{E}-03$ & & $4.13 \mathrm{E}-03$ & \\
\hline Ce-144 & $7.60 \mathrm{E}-02$ & $7.58 \mathrm{E}-02$ & & $1.85 \mathrm{E}-02$ & \\
\hline Pm-147 & $6.60 \mathrm{E}-03$ & $7.50 \mathrm{E}-03$ & $\checkmark$ & $1.83 \mathrm{E}-03$ & \\
\hline Eu-152 & $1.50 \mathrm{E}+00$ & $2.54 \mathrm{E}+00$ & $\checkmark$ & $5.97 \mathrm{E}-01$ & \\
\hline Eu-154 & $1.20 \mathrm{E}+00$ & $2.05 \mathrm{E}+00$ & $\checkmark$ & $4.82 \mathrm{E}-01$ & \\
\hline Eu-155 & $5.00 \mathrm{E}-02$ & $7.91 \mathrm{E}-02$ & $\checkmark$ & $1.87 \mathrm{E}-02$ & \\
\hline Tl-208 & $1.10 \mathrm{E}-04$ & $1.98 \mathrm{E}-04$ & $\checkmark$ & 4.52E-05 & \\
\hline $\mathrm{Pb}-212$ & $2.20 \mathrm{E}-02$ & $3.27 \mathrm{E}-02$ & $\checkmark$ & $7.87 \mathrm{E}-03$ & \\
\hline Bi-212 & $4.20 \mathrm{E}-03$ & $6.33 \mathrm{E}-03$ & $\checkmark$ & $1.47 \mathrm{E}-03$ & \\
\hline U-234 & $1.90 \mathrm{E}+01$ & $2.53 \mathrm{E}+01$ & $\checkmark$ & $6.17 \mathrm{E}+00$ & \\
\hline $\mathrm{U}-235$ & $1.80 \mathrm{E}+01$ & $2.43 \mathrm{E}+01$ & $\checkmark$ & $5.92 \mathrm{E}+00$ & \\
\hline U-238 & $1.70 \mathrm{E}+01$ & $2.25 \mathrm{E}+01$ & $\checkmark$ & $5.49 \mathrm{E}+00$ & \\
\hline $\mathrm{Pu}-239$ & $5.00 \mathrm{E}+01$ & $6.55 \mathrm{E}+01$ & $\checkmark$ & $1.60 \mathrm{E}+01$ & \\
\hline $\mathrm{Pu}-241$ & $7.90 \mathrm{E}-01$ & $9.96 \mathrm{E}-01$ & $\checkmark$ & $2.43 \mathrm{E}-01$ & \\
\hline Am-241 & $7.70 \mathrm{E}+01$ & $1.04 \mathrm{E}+02$ & $\checkmark$ & $2.54 \mathrm{E}+01$ & \\
\hline \multicolumn{6}{|c|}{$\begin{array}{l}\text { (a) Offsite receptor at } 1400 \mathrm{~m} \text { NE (HNF-3602, Hill and Rittmann 1999) } \\
\text { (b) Onsite receptor at } 313 \text { Building (Kaiser), } 540 \mathrm{~m} \mathrm{NW} \text {. } \\
\text { (c) Assumes } 8766 \mathrm{~h} / \mathrm{y} \text { occupancy onsite for compliance with EPA regulations. Includes dose from } \\
\text { ingestion of food produced regionally. } \\
\text { (d) Assumes } 2000 \mathrm{~h} / \mathrm{y} \text { occupancy onsite for compliance with DOE standards. Includes dose from ingestion } \\
\text { of food produced regionally and exposure via inhalation and external pathways typical of an "average" } \\
\text { member of the population for remainder of year }(6766 \mathrm{~h} / \mathrm{y}) \text {. }\end{array}$} \\
\hline
\end{tabular}


Table F.13. Unit Dose Factors for 327 Bldg for 10-m Releases - Total Dose to Individual, mrem/y per $\mathrm{Ci} / \mathrm{y}$ Release

\begin{tabular}{|c|c|c|c|c|c|}
\hline Nuclide & ${\text { Offsite } M E I^{(a)}}^{(a)}$ & $\begin{array}{c}\text { Onsite } \\
\text { Receptor }^{(b)} \text { Full- } \\
\text { time Exposure }^{(\text {c) }}\end{array}$ & $\begin{array}{c}\text { Onsite }> \\
\text { Offsite }\end{array}$ & $\begin{array}{c}\text { Onsite Receptor }^{(b)} \\
\text { Working-Year }^{\left(\text {Exposure }^{(d)}\right.}\end{array}$ & $\begin{array}{c}\text { Onsite }> \\
\text { Offsite }\end{array}$ \\
\hline $\mathrm{H}-3$ & $3.40 \mathrm{E}-04$ & $3.82 \mathrm{E}-04$ & $\checkmark$ & $9.30 \mathrm{E}-05$ & \\
\hline $\mathrm{C}-14$ & $2.70 \mathrm{E}-02$ & $4.75 \mathrm{E}-04$ & & $4.48 \mathrm{E}-04$ & \\
\hline $\mathrm{O}-15$ & $4.30 \mathrm{E}-05$ & $8.35 \mathrm{E}-04$ & $\checkmark$ & $1.91 \mathrm{E}-04$ & $\checkmark$ \\
\hline Ar-41 & $2.80 \mathrm{E}-04$ & $2.16 \mathrm{E}-03$ & $\checkmark$ & $4.95 \mathrm{E}-04$ & $\checkmark$ \\
\hline Mn-54 & $2.70 \mathrm{E}-01$ & $1.97 \mathrm{E}+00$ & $\checkmark$ & $4.51 \mathrm{E}-01$ & $\checkmark$ \\
\hline Co-60 & $4.10 \mathrm{E}+00$ & $2.95 \mathrm{E}+01$ & $\checkmark$ & $6.76 \mathrm{E}+00$ & $\checkmark$ \\
\hline $\mathrm{Zn}-65$ & $4.10 \mathrm{E}-01$ & $1.06 \mathrm{E}+00$ & $\checkmark$ & $2.45 \mathrm{E}-01$ & \\
\hline $\mathrm{Zn}-69 \mathrm{~m}$ & $7.60 \mathrm{E}-04$ & $5.49 \mathrm{E}-03$ & $\checkmark$ & $1.26 \mathrm{E}-03$ & $\checkmark$ \\
\hline Kr-85 & $7.70 \mathrm{E}-07$ & $5.58 \mathrm{E}-06$ & $\checkmark$ & $1.29 \mathrm{E}-06$ & $\checkmark$ \\
\hline Sr-90 & $1.90 \mathrm{E}+00$ & $6.34 \mathrm{E}-01$ & & $1.54 \mathrm{E}-01$ & \\
\hline Zr-95 & $6.50 \mathrm{E}-02$ & $4.03 \mathrm{E}-01$ & $\checkmark$ & $9.23 \mathrm{E}-02$ & $\checkmark$ \\
\hline $\mathrm{Nb}-95$ & $8.00 \mathrm{E}-02$ & $2.24 \mathrm{E}-01$ & $\checkmark$ & $5.17 \mathrm{E}-02$ & \\
\hline Ru-103 & $3.10 \mathrm{E}-02$ & $1.75 \mathrm{E}-01$ & $\checkmark$ & $4.01 \mathrm{E}-02$ & $\checkmark$ \\
\hline $\mathrm{Ru}-106$ & $2.70 \mathrm{E}-01$ & $1.34 \mathrm{E}+00$ & $\checkmark$ & $3.08 \mathrm{E}-01$ & $\checkmark$ \\
\hline Ag-110 & $8.50 \mathrm{E}-09$ & $4.33 \mathrm{E}-06$ & $\checkmark$ & $9.88 \mathrm{E}-07$ & $\checkmark$ \\
\hline Sn-113 & $3.70 \mathrm{E}-02$ & 4.49E-02 & $\checkmark$ & $1.05 \mathrm{E}-02$ & \\
\hline $\mathrm{Sb}-125$ & $4.40 \mathrm{E}-01$ & $3.18 \mathrm{E}+00$ & $\checkmark$ & $7.28 \mathrm{E}-01$ & $\checkmark$ \\
\hline $\mathrm{I}-129$ & $3.60 \mathrm{E}+01$ & $2.28 \mathrm{E}+01$ & & $5.23 \mathrm{E}+00$ & \\
\hline $\mathrm{I}-131$ & $1.20 \mathrm{E}+00$ & $5.04 \mathrm{E}-01$ & & $1.15 \mathrm{E}-01$ & \\
\hline Cs-134 & $1.70 \mathrm{E}+00$ & $8.65 \mathrm{E}+00$ & $\checkmark$ & $1.98 \mathrm{E}+00$ & $\checkmark$ \\
\hline Cs-137+D & $3.97 \mathrm{E}+00$ & $2.65 \mathrm{E}+01$ & $\checkmark$ & $6.07 \mathrm{E}+00$ & $\checkmark$ \\
\hline Cs-137 & $4.40 \mathrm{E}-01$ & $9.06 \mathrm{E}-02$ & & $2.36 \mathrm{E}-02$ & \\
\hline Ce-144 & $2.10 \mathrm{E}-01$ & $1.10 \mathrm{E}+00$ & $\checkmark$ & $2.53 \mathrm{E}-01$ & $\checkmark$ \\
\hline Pm-147 & $1.80 \mathrm{E}-02$ & $1.10 \mathrm{E}-01$ & $\checkmark$ & $2.53 \mathrm{E}-02$ & $\checkmark$ \\
\hline Eu-152 & $4.00 \mathrm{E}+00$ & $3.00 \mathrm{E}+01$ & $\checkmark$ & $6.87 \mathrm{E}+00$ & $\checkmark$ \\
\hline Eu-154 & $3.30 \mathrm{E}+00$ & $2.42 \mathrm{E}+01$ & $\checkmark$ & $5.54 \mathrm{E}+00$ & $\checkmark$ \\
\hline Eu-155 & $1.30 \mathrm{E}-01$ & $9.53 \mathrm{E}-01$ & $\checkmark$ & $2.18 \mathrm{E}-01$ & $\checkmark$ \\
\hline T1-208 & $2.60 \mathrm{E}-04$ & $4.01 \mathrm{E}-03$ & $\checkmark$ & $9.15 \mathrm{E}-04$ & $\checkmark$ \\
\hline $\mathrm{Pb}-212$ & $6.40 \mathrm{E}-02$ & 4.79E-01 & $\checkmark$ & $1.10 \mathrm{E}-01$ & $\checkmark$ \\
\hline Bi-212 & $1.20 \mathrm{E}-02$ & $9.46 \mathrm{E}-02$ & $\checkmark$ & $2.16 \mathrm{E}-02$ & $\checkmark$ \\
\hline U-234 & $5.30 \mathrm{E}+01$ & $3.71 \mathrm{E}+02$ & $\checkmark$ & $8.52 \mathrm{E}+01$ & $\checkmark$ \\
\hline U-235 & $5.10 \mathrm{E}+01$ & $3.54 \mathrm{E}+02$ & $\checkmark$ & $8.13 \mathrm{E}+01$ & $\checkmark$ \\
\hline U-238 & $4.70 \mathrm{E}+01$ & $3.30 \mathrm{E}+02$ & $\checkmark$ & $7.58 \mathrm{E}+01$ & $\checkmark$ \\
\hline $\mathrm{Pu}-239$ & $1.40 \mathrm{E}+02$ & $9.59 \mathrm{E}+02$ & $\checkmark$ & $2.20 \mathrm{E}+02$ & $\checkmark$ \\
\hline $\mathrm{Pu}-241$ & $2.20 \mathrm{E}+00$ & $1.46 \mathrm{E}+01$ & $\checkmark$ & $3.35 \mathrm{E}+00$ & $\checkmark$ \\
\hline Am-241 & $2.20 \mathrm{E}+02$ & $1.53 \mathrm{E}+03$ & $\checkmark$ & $3.51 \mathrm{E}+02$ & $\checkmark$ \\
\hline \multicolumn{6}{|c|}{$\begin{array}{l}\text { (a) Offsite receptor at } 1400 \mathrm{~m} \text { NE (HNF-3602, Hill and Rittmann 1999). } \\
\text { (b) Onsite receptor at N } 313 \text { Building (Kaiser), } 250 \mathrm{~m} \text { NNW. } \\
\text { (c) Assumes } 8766 \mathrm{~h} / \mathrm{y} \text { occupancy onsite for compliance with EPA regulations. Includes dose from ingestion } \\
\text { of food produced regionally. } \\
\text { (d) Assumes } 2000 \mathrm{~h} / \mathrm{y} \text { occupancy onsite for compliance with DOE standards. Includes dose from ingestion } \\
\text { of food produced regionally and exposure via inhalation and external pathways typical of an "average" } \\
\text { member of the population for remainder of year }(6766 \mathrm{~h} / \mathrm{y}) .\end{array}$} \\
\hline
\end{tabular}


Table F.14. Unit Dose Factors for 331 Bldg for 10-m Releases - Total Dose to Individual, $\mathrm{mrem} / \mathrm{y}$ per $\mathrm{Ci} / \mathrm{y}$ Release

\begin{tabular}{|c|c|c|c|c|c|}
\hline Nuclide & Offsite MEI $^{(\mathbf{a})}$ & $\begin{array}{l}\text { Onsite } \\
\text { Receptor }^{(b)} \text { Full- } \\
\text { time Exposure }^{(c)}\end{array}$ & $\begin{array}{c}\text { Onsite > } \\
\text { Offsite }\end{array}$ & $\begin{array}{c}\text { Onsite Receptor }^{(\mathbf{b})} \\
\text { Working-Year }^{\text {(d) }} \\
\text { Exposure }^{(\mathbf{d})}\end{array}$ & $\begin{array}{c}\text { Onsite }> \\
\text { Offsite }\end{array}$ \\
\hline $\mathrm{H}-3$ & 4.80E-04 & $1.33 \mathrm{E}-04$ & & $3.61 \mathrm{E}-05$ & \\
\hline C-14 & $3.70 \mathrm{E}-02$ & $4.52 \mathrm{E}-04$ & & $4.43 \mathrm{E}-04$ & \\
\hline $\mathrm{O}-15$ & $8.10 \mathrm{E}-05$ & $6.97 \mathrm{E}-05$ & & $1.59 \mathrm{E}-05$ & \\
\hline Ar-41 & 4.00E-04 & 7.04E-04 & $\checkmark$ & $1.63 \mathrm{E}-04$ & \\
\hline Mn-54 & $3.80 \mathrm{E}-01$ & $6.53 \mathrm{E}-01$ & $\checkmark$ & $1.51 \mathrm{E}-01$ & \\
\hline Co-60 & $5.70 \mathrm{E}+00$ & $9.77 \mathrm{E}+00$ & $\checkmark$ & $2.25 \mathrm{E}+00$ & \\
\hline $\mathrm{Zn}-65$ & $5.70 \mathrm{E}-01$ & $3.53 \mathrm{E}-01$ & & $8.34 \mathrm{E}-02$ & \\
\hline $\mathrm{Zn}-69 \mathrm{~m}$ & $1.10 \mathrm{E}-03$ & $1.80 \mathrm{E}-03$ & $\checkmark$ & $4.17 \mathrm{E}-04$ & \\
\hline $\mathrm{Kr}-85$ & $1.10 \mathrm{E}-06$ & $1.88 \mathrm{E}-06$ & $\checkmark$ & $4.42 \mathrm{E}-07$ & \\
\hline Sr-90 & $2.60 \mathrm{E}+00$ & $2.16 \mathrm{E}-01$ & & $5.84 \mathrm{E}-02$ & \\
\hline Zr-95 & $9.10 \mathrm{E}-02$ & $1.33 \mathrm{E}-01$ & $\checkmark$ & $3.07 \mathrm{E}-02$ & \\
\hline $\mathrm{Nb}-95$ & $1.10 \mathrm{E}-01$ & $7.46 \mathrm{E}-02$ & & $1.75 \mathrm{E}-02$ & \\
\hline Ru-103 & 4.30E-02 & 5.79E-02 & $\checkmark$ & $1.34 \mathrm{E}-02$ & \\
\hline $\mathrm{Ru}-106$ & $3.70 \mathrm{E}-01$ & $4.44 \mathrm{E}-01$ & $\checkmark$ & $1.04 \mathrm{E}-01$ & \\
\hline Ag-110 & 4.80E-08 & $1.98 \mathrm{E}-08$ & & $4.52 \mathrm{E}-09$ & \\
\hline Sn-113 & $5.10 \mathrm{E}-02$ & $1.50 \mathrm{E}-02$ & & $3.68 \mathrm{E}-03$ & \\
\hline Sb-125 & $6.10 \mathrm{E}-01$ & $1.05 \mathrm{E}+00$ & $\checkmark$ & $2.42 \mathrm{E}-01$ & \\
\hline $\mathrm{I}-129$ & $5.60 \mathrm{E}+01$ & $5.33 \mathrm{E}+00$ & & $1.24 \mathrm{E}+00$ & \\
\hline $\mathrm{I}-131$ & $1.80 \mathrm{E}+00$ & $1.18 \mathrm{E}-01$ & & $2.74 \mathrm{E}-02$ & \\
\hline Cs-134 & $2.30 \mathrm{E}+00$ & $2.86 \mathrm{E}+00$ & $\checkmark$ & $6.64 \mathrm{E}-01$ & \\
\hline Cs-137+D & $5.59 \mathrm{E}+00$ & $8.75 \mathrm{E}+00$ & $\checkmark$ & $2.02 \mathrm{E}+00$ & \\
\hline Cs-137 & $6.20 \mathrm{E}-01$ & $3.24 \mathrm{E}-02$ & & $1.03 \mathrm{E}-02$ & \\
\hline $\mathrm{Ce}-144$ & $2.90 \mathrm{E}-01$ & $3.63 \mathrm{E}-01$ & $\checkmark$ & $8.46 \mathrm{E}-02$ & \\
\hline Pm-147 & $2.60 \mathrm{E}-02$ & $3.63 \mathrm{E}-02$ & $\checkmark$ & $8.46 \mathrm{E}-03$ & \\
\hline Eu-152 & $5.60 \mathrm{E}+00$ & $9.92 \mathrm{E}+00$ & $\checkmark$ & $2.29 \mathrm{E}+00$ & \\
\hline Eu-154 & $4.60 \mathrm{E}+00$ & $8.01 \mathrm{E}+00$ & $\checkmark$ & $1.85 \mathrm{E}+00$ & \\
\hline Eu-155 & $1.90 \mathrm{E}-01$ & $3.15 \mathrm{E}-01$ & $\checkmark$ & $7.27 \mathrm{E}-02$ & \\
\hline T1-208 & $4.40 \mathrm{E}-04$ & $4.93 \mathrm{E}-04$ & $\checkmark$ & $1.13 \mathrm{E}-04$ & \\
\hline $\mathrm{Pb}-212$ & $8.90 \mathrm{E}-02$ & $1.57 \mathrm{E}-01$ & $\checkmark$ & $3.64 \mathrm{E}-02$ & \\
\hline $\mathrm{Bi}-212$ & $1.70 \mathrm{E}-02$ & 2.94E-02 & $\checkmark$ & $6.75 \mathrm{E}-03$ & \\
\hline $\mathrm{U}-234$ & $7.40 \mathrm{E}+01$ & $1.23 \mathrm{E}+02$ & $\checkmark$ & $2.86 \mathrm{E}+01$ & \\
\hline U-235 & $7.10 \mathrm{E}+01$ & $1.17 \mathrm{E}+02$ & $\checkmark$ & $2.72 \mathrm{E}+01$ & \\
\hline $\mathrm{U}-238$ & $6.60 \mathrm{E}+01$ & $1.09 \mathrm{E}+02$ & $\checkmark$ & $2.54 \mathrm{E}+01$ & \\
\hline $\mathrm{Pu}-239$ & $2.00 \mathrm{E}+02$ & $3.18 \mathrm{E}+02$ & $\checkmark$ & $7.40 \mathrm{E}+01$ & \\
\hline $\mathrm{Pu}-241$ & $3.10 \mathrm{E}+00$ & $4.83 \mathrm{E}+00$ & $\checkmark$ & $1.12 \mathrm{E}+00$ & \\
\hline Am-241 & $3.00 \mathrm{E}+02$ & $5.05 \mathrm{E}+02$ & $\checkmark$ & $1.18 \mathrm{E}+02$ & \\
\hline \multicolumn{6}{|c|}{$\begin{array}{l}\text { (a) Offsite receptor at } 1100 \mathrm{~m} \text { NE (HNF-3602, Hill and Rittmann 1999) } \\
\text { (b) Onsite receptor at } 3746 \text { Building (WSU), } 980 \mathrm{~m} \mathrm{NW} \text {. } \\
\text { (c) Assumes } 8766 \mathrm{~h} / \mathrm{y} \text { occupancy onsite for compliance with EPA regulations. Includes dose from ingestion } \\
\text { of food produced regionally. } \\
\text { (d) Assumes } 2000 \mathrm{~h} / \mathrm{y} \text { occupancy onsite for compliance with DOE standards. Includes dose from ingestion } \\
\text { of food produced regionally and exposure via inhalation and external pathways typical of an "average" } \\
\text { member of the population for remainder of year }(6766 \mathrm{~h} / \mathrm{y}) \text {. }\end{array}$} \\
\hline
\end{tabular}


Table F.15. Unit Dose Factors for 340 Bldg for 10-m Releases - Total Dose to Individual, $\mathrm{mrem} / \mathrm{y}$ per $\mathrm{Ci} / \mathrm{y}$ Release

\begin{tabular}{|c|c|c|c|c|c|}
\hline Nuclide & Offsite $M E I^{(a)}$ & $\begin{array}{c}\text { Onsite } \\
\text { Receptor }^{(b)} \text { Full- } \\
\text { time Exposure }^{(\mathrm{c})}\end{array}$ & $\begin{array}{c}\text { Onsite > } \\
\text { Offsite }\end{array}$ & $\begin{array}{c}\text { Onsite Receptor }^{(\mathbf{b})} \\
\text { Working-Year }^{(\mathrm{d})} \\
\text { Exposure }^{(\mathrm{s})}\end{array}$ & $\begin{array}{c}\text { Onsite }> \\
\text { Offsite }\end{array}$ \\
\hline $\mathrm{H}-3$ & $3.40 \mathrm{E}-04$ & $3.66 \mathrm{E}-04$ & $\checkmark$ & $8.93 \mathrm{E}-05$ & \\
\hline C-14 & $2.70 \mathrm{E}-02$ & $4.73 \mathrm{E}-04$ & & $4.48 \mathrm{E}-04$ & \\
\hline $\mathrm{O}-15$ & $4.30 \mathrm{E}-05$ & $6.46 \mathrm{E}-04$ & $\checkmark$ & $1.47 \mathrm{E}-04$ & $\checkmark$ \\
\hline Ar-41 & $2.80 \mathrm{E}-04$ & $2.06 \mathrm{E}-03$ & $\checkmark$ & 4.72E-04 & $\checkmark$ \\
\hline $\mathrm{Mn}-54$ & $2.70 \mathrm{E}-01$ & $1.88 \mathrm{E}+00$ & $\checkmark$ & $4.31 \mathrm{E}-01$ & $\checkmark$ \\
\hline Co-60 & $4.10 \mathrm{E}+00$ & $2.81 \mathrm{E}+01$ & $\checkmark$ & $6.44 \mathrm{E}+00$ & $\checkmark$ \\
\hline $\mathrm{Zn}-65$ & $4.10 \mathrm{E}-01$ & $1.01 \mathrm{E}+00$ & $\checkmark$ & $2.34 \mathrm{E}-01$ & \\
\hline $\mathrm{Zn}-69 \mathrm{~m}$ & $7.60 \mathrm{E}-04$ & $5.23 \mathrm{E}-03$ & $\checkmark$ & $1.20 \mathrm{E}-03$ & $\checkmark$ \\
\hline $\mathrm{Kr}-85$ & $7.70 \mathrm{E}-07$ & $5.34 \mathrm{E}-06$ & $\checkmark$ & $1.23 \mathrm{E}-06$ & $\checkmark$ \\
\hline Sr-90 & $1.90 \mathrm{E}+00$ & $6.05 \mathrm{E}-01$ & & $1.47 \mathrm{E}-01$ & \\
\hline $\mathrm{Zr}-95$ & $6.50 \mathrm{E}-02$ & $3.84 \mathrm{E}-01$ & $\checkmark$ & $8.80 \mathrm{E}-02$ & $\checkmark$ \\
\hline $\mathrm{Nb}-95$ & $8.00 \mathrm{E}-02$ & $2.13 \mathrm{E}-01$ & $\checkmark$ & 4.92E-02 & \\
\hline Ru-103 & $3.10 \mathrm{E}-02$ & $1.67 \mathrm{E}-01$ & $\checkmark$ & $3.83 \mathrm{E}-02$ & $\checkmark$ \\
\hline Ru-106 & $2.70 \mathrm{E}-01$ & $1.28 \mathrm{E}+00$ & $\checkmark$ & $2.94 \mathrm{E}-01$ & $\checkmark$ \\
\hline Ag-110 & 8.50E-09 & $1.61 \mathrm{E}-06$ & $\checkmark$ & $3.67 \mathrm{E}-07$ & $\checkmark$ \\
\hline Sn-113 & $3.70 \mathrm{E}-02$ & $4.28 \mathrm{E}-02$ & $\checkmark$ & $1.00 \mathrm{E}-02$ & \\
\hline Sb-125 & $4.40 \mathrm{E}-01$ & $3.02 \mathrm{E}+00$ & $\checkmark$ & $6.92 \mathrm{E}-01$ & $\checkmark$ \\
\hline $\mathrm{I}-129$ & $3.60 \mathrm{E}+01$ & $2.08 \mathrm{E}+01$ & & $4.77 \mathrm{E}+00$ & \\
\hline $\mathrm{I}-131$ & $1.20 \mathrm{E}+00$ & 4.59E-01 & & $1.05 \mathrm{E}-01$ & \\
\hline Cs-134 & $1.70 \mathrm{E}+00$ & $8.23 \mathrm{E}+00$ & $\checkmark$ & $1.89 \mathrm{E}+00$ & $\checkmark$ \\
\hline Cs-137+D & $3.97 \mathrm{E}+00$ & $2.52 \mathrm{E}+01$ & $\checkmark$ & $5.77 \mathrm{E}+00$ & $\checkmark$ \\
\hline Cs-137 & 4.40E-01 & $8.66 \mathrm{E}-02$ & & $2.27 \mathrm{E}-02$ & \\
\hline Ce-144 & $2.10 \mathrm{E}-01$ & $1.05 \mathrm{E}+00$ & $\checkmark$ & $2.41 \mathrm{E}-01$ & $\checkmark$ \\
\hline Pm-147 & $1.80 \mathrm{E}-02$ & $1.05 \mathrm{E}-01$ & $\checkmark$ & $2.41 \mathrm{E}-02$ & $\checkmark$ \\
\hline Eu-152 & $4.00 \mathrm{E}+00$ & $2.85 \mathrm{E}+01$ & $\checkmark$ & $6.53 \mathrm{E}+00$ & $\checkmark$ \\
\hline Eu-154 & $3.30 \mathrm{E}+00$ & $2.30 \mathrm{E}+01$ & $\checkmark$ & $5.27 \mathrm{E}+00$ & $\checkmark$ \\
\hline Eu-155 & $1.30 \mathrm{E}-01$ & $9.06 \mathrm{E}-01$ & $\checkmark$ & $2.08 \mathrm{E}-01$ & $\checkmark$ \\
\hline Tl-208 & $2.60 \mathrm{E}-04$ & $3.30 \mathrm{E}-03$ & $\checkmark$ & $7.53 \mathrm{E}-04$ & $\checkmark$ \\
\hline $\mathrm{Pb}-212$ & $6.40 \mathrm{E}-02$ & $4.57 \mathrm{E}-01$ & $\checkmark$ & $1.05 \mathrm{E}-01$ & $\checkmark$ \\
\hline Bi-212 & $1.20 \mathrm{E}-02$ & $8.95 \mathrm{E}-02$ & $\checkmark$ & $2.05 \mathrm{E}-02$ & $\checkmark$ \\
\hline U-234 & $5.30 \mathrm{E}+01$ & $3.54 \mathrm{E}+02$ & $\checkmark$ & $8.13 \mathrm{E}+01$ & $\checkmark$ \\
\hline U-235 & $5.10 \mathrm{E}+01$ & $3.37 \mathrm{E}+02$ & $\checkmark$ & $7.74 \mathrm{E}+01$ & $\checkmark$ \\
\hline U-238 & $4.70 \mathrm{E}+01$ & $3.15 \mathrm{E}+02$ & $\checkmark$ & $7.24 \mathrm{E}+01$ & $\checkmark$ \\
\hline $\mathrm{Pu}-239$ & $1.40 \mathrm{E}+02$ & $9.16 \mathrm{E}+02$ & $\checkmark$ & $2.10 \mathrm{E}+02$ & $\checkmark$ \\
\hline $\mathrm{Pu}-241$ & $2.20 \mathrm{E}+00$ & $1.39 \mathrm{E}+01$ & $\checkmark$ & $3.19 \mathrm{E}+00$ & $\checkmark$ \\
\hline Am-241 & $2.20 \mathrm{E}+02$ & $1.46 \mathrm{E}+03$ & $\checkmark$ & $3.35 \mathrm{E}+02$ & $\checkmark$ \\
\hline \multicolumn{6}{|c|}{$\begin{array}{l}\text { (a) Offsite receptor at } 1400 \mathrm{~m} \mathrm{NE} \text { (HNF-3602, Hill and Rittmann 1999). } \\
\text { (b) Onsite receptor at } 313 \text { Building (Kaiser), } 385 \mathrm{~m} \mathrm{NW} \text {. } \\
\text { (c) Assumes } 8766 \mathrm{~h} / \mathrm{y} \text { occupancy onsite for compliance with EPA regulations. Includes dose from ingestion } \\
\text { of food produced regionally. } \\
\text { (d) Assumes } 2000 \mathrm{~h} / \mathrm{y} \text { occupancy onsite for compliance with DOE standards. Includes dose from ingestion } \\
\text { of food produced regionally and exposure via inhalation and external pathways typical of an "average" } \\
\text { member of the population for remainder of year }(6766 \mathrm{~h} / \mathrm{y}) .\end{array}$} \\
\hline
\end{tabular}


Table F.16. Unit Dose Factors for 3720 Bldg for 10-m Releases - Total Dose to Individual, $\mathrm{mrem} / \mathrm{y}$ per $\mathrm{Ci} / \mathrm{y}$ Release

\begin{tabular}{|c|c|c|c|c|c|}
\hline Nuclide & Offsite $M E I^{(a)}$ & $\begin{array}{c}\text { Onsite } \\
\text { Receptor }^{(b)} \text { Full- } \\
\text { time Exposure }^{(\mathrm{c})}\end{array}$ & $\begin{array}{c}\text { Onsite }> \\
\text { Offsite }\end{array}$ & $\begin{array}{c}\text { Onsite Receptor }^{(b)} \\
\text { Working-Year }^{(\mathbf{b})} \\
\text { Exposure }^{(\mathrm{d})}\end{array}$ & $\begin{array}{c}\text { Onsite }> \\
\text { Offsite }\end{array}$ \\
\hline $\mathrm{H}-3$ & $3.40 \mathrm{E}-04$ & $3.94 \mathrm{E}-04$ & $\checkmark$ & $9.57 \mathrm{E}-05$ & \\
\hline $\mathrm{C}-14$ & $2.70 \mathrm{E}-02$ & $4.76 \mathrm{E}-04$ & & $4.48 \mathrm{E}-04$ & \\
\hline $\mathrm{O}-15$ & $4.30 \mathrm{E}-05$ & $1.55 \mathrm{E}-03$ & $\checkmark$ & $3.54 \mathrm{E}-04$ & $\checkmark$ \\
\hline Ar-41 & $2.80 \mathrm{E}-04$ & $2.25 \mathrm{E}-03$ & $\checkmark$ & $5.15 \mathrm{E}-04$ & $\checkmark$ \\
\hline Mn-54 & $2.70 \mathrm{E}-01$ & $2.09 \mathrm{E}+00$ & $\checkmark$ & $4.78 \mathrm{E}-01$ & $\checkmark$ \\
\hline Co-60 & $4.10 \mathrm{E}+00$ & $3.13 \mathrm{E}+01$ & $\checkmark$ & $7.17 \mathrm{E}+00$ & $\checkmark$ \\
\hline Zn-65 & $4.10 \mathrm{E}-01$ & $1.12 \mathrm{E}+00$ & $\checkmark$ & $2.59 \mathrm{E}-01$ & \\
\hline $\mathrm{Zn}-69 \mathrm{~m}$ & $7.60 \mathrm{E}-04$ & $5.73 \mathrm{E}-03$ & $\checkmark$ & $1.31 \mathrm{E}-03$ & $\checkmark$ \\
\hline $\mathrm{Kr}-85$ & $7.70 \mathrm{E}-07$ & $5.75 \mathrm{E}-06$ & $\checkmark$ & $1.32 \mathrm{E}-06$ & $\checkmark$ \\
\hline Sr-90 & $1.90 \mathrm{E}+00$ & $6.56 \mathrm{E}-01$ & & $1.59 \mathrm{E}-01$ & \\
\hline Zr-95 & $6.50 \mathrm{E}-02$ & $4.27 \mathrm{E}-01$ & $\checkmark$ & $9.78 \mathrm{E}-02$ & $\checkmark$ \\
\hline $\mathrm{Nb}-95$ & $8.00 \mathrm{E}-02$ & $2.37 \mathrm{E}-01$ & $\checkmark$ & $5.47 \mathrm{E}-02$ & \\
\hline Ru-103 & $3.10 \mathrm{E}-02$ & $1.85 \mathrm{E}-01$ & $\checkmark$ & 4.24E-02 & $\checkmark$ \\
\hline Ru-106 & $2.70 \mathrm{E}-01$ & $1.39 \mathrm{E}+00$ & $\checkmark$ & $3.19 \mathrm{E}-01$ & $\checkmark$ \\
\hline Ag-110 & $8.50 \mathrm{E}-09$ & $3.33 \mathrm{E}-05$ & $\checkmark$ & 7.60E-06 & $\checkmark$ \\
\hline Sn-113 & $3.70 \mathrm{E}-02$ & $4.68 \mathrm{E}-02$ & $\checkmark$ & 1.09E-02 & \\
\hline Sb-125 & $4.40 \mathrm{E}-01$ & $3.37 \mathrm{E}+00$ & $\checkmark$ & $7.71 \mathrm{E}-01$ & $\checkmark$ \\
\hline $\mathrm{I}-129$ & $3.60 \mathrm{E}+01$ & $2.58 \mathrm{E}+01$ & & $5.91 \mathrm{E}+00$ & \\
\hline $\mathrm{I}-131$ & $1.20 \mathrm{E}+00$ & 5.70E-01 & & $1.30 \mathrm{E}-01$ & \\
\hline Cs-134 & $1.70 \mathrm{E}+00$ & $9.18 \mathrm{E}+00$ & $\checkmark$ & $2.11 \mathrm{E}+00$ & $\checkmark$ \\
\hline Cs-137+D & $3.97 \mathrm{E}+00$ & $2.81 \mathrm{E}+01$ & $\checkmark$ & $6.43 \mathrm{E}+00$ & $\checkmark$ \\
\hline Cs-137 & 4.40E-01 & $9.37 \mathrm{E}-02$ & & $2.43 \mathrm{E}-02$ & \\
\hline Ce-144 & $2.10 \mathrm{E}-01$ & $1.14 \mathrm{E}+00$ & $\checkmark$ & $2.62 \mathrm{E}-01$ & $\checkmark$ \\
\hline Pm-147 & $1.80 \mathrm{E}-02$ & $1.13 \mathrm{E}-01$ & $\checkmark$ & $2.60 \mathrm{E}-02$ & $\checkmark$ \\
\hline Eu-152 & $4.00 \mathrm{E}+00$ & $3.18 \mathrm{E}+01$ & $\checkmark$ & $7.28 \mathrm{E}+00$ & $\checkmark$ \\
\hline Eu-154 & $3.30 \mathrm{E}+00$ & $2.57 \mathrm{E}+01$ & $\checkmark$ & $5.88 \mathrm{E}+00$ & $\checkmark$ \\
\hline Eu-155 & $1.30 \mathrm{E}-01$ & $1.01 \mathrm{E}+00$ & $\checkmark$ & $2.31 \mathrm{E}-01$ & $\checkmark$ \\
\hline Tl-208 & $2.60 \mathrm{E}-04$ & $6.24 \mathrm{E}-03$ & $\checkmark$ & $1.42 \mathrm{E}-03$ & $\checkmark$ \\
\hline $\mathrm{Pb}-212$ & $6.40 \mathrm{E}-02$ & $4.96 \mathrm{E}-01$ & $\checkmark$ & $1.14 \mathrm{E}-01$ & $\checkmark$ \\
\hline Bi-212 & $1.20 \mathrm{E}-02$ & $1.00 \mathrm{E}-01$ & $\checkmark$ & $2.29 \mathrm{E}-02$ & $\checkmark$ \\
\hline U-234 & $5.30 \mathrm{E}+01$ & $3.84 \mathrm{E}+02$ & $\checkmark$ & $8.82 \mathrm{E}+01$ & $\checkmark$ \\
\hline $\mathrm{U}-235$ & $5.10 \mathrm{E}+01$ & $3.66 \mathrm{E}+02$ & $\checkmark$ & $8.40 \mathrm{E}+01$ & $\checkmark$ \\
\hline U-238 & $4.70 \mathrm{E}+01$ & $3.42 \mathrm{E}+02$ & $\checkmark$ & $7.85 \mathrm{E}+01$ & $\checkmark$ \\
\hline $\mathrm{Pu}-239$ & $1.40 \mathrm{E}+02$ & $9.93 \mathrm{E}+02$ & $\checkmark$ & $2.28 \mathrm{E}+02$ & $\checkmark$ \\
\hline $\mathrm{Pu}-241$ & $2.20 \mathrm{E}+00$ & $1.51 \mathrm{E}+01$ & $\checkmark$ & $3.47 \mathrm{E}+00$ & $\checkmark$ \\
\hline Am-241 & $2.20 \mathrm{E}+02$ & $1.58 \mathrm{E}+03$ & $\checkmark$ & $3.63 \mathrm{E}+02$ & $\checkmark$ \\
\hline \multicolumn{6}{|c|}{$\begin{array}{l}\text { (a) Offsite receptor at } 1400 \mathrm{~m} \mathrm{NE} \text { (HNF-3602, Hill and Rittmann 1999). } \\
\text { (b) Onsite receptor at } 313 \text { Building (Kaiser), } 70 \mathrm{~m} \text { SSW. } \\
\text { (c) Assumes } 8766 \mathrm{~h} / \mathrm{y} \text { occupancy onsite for compliance with EPA regulations. Includes dose from ingestion } \\
\text { of food produced regionally. } \\
\text { (d) Assumes } 2000 \mathrm{~h} / \mathrm{y} \text { occupancy onsite for compliance with DOE standards. Includes dose from ingestion } \\
\text { of food produced regionally and exposure via inhalation and external pathways typical of an "average" } \\
\text { member of the population for remainder of year }(6766 \mathrm{~h} / \mathrm{y}) .\end{array}$} \\
\hline
\end{tabular}


Table F.17. Unit Dose Factors for 400 Area for 10-m Releases - Total Dose to Individual, $\mathrm{mrem} / \mathrm{y}$ per $\mathrm{Ci} / \mathrm{y}$ Release

\begin{tabular}{|c|c|c|c|c|c|}
\hline Nuclide & Offsite MEI ${ }^{(\mathbf{a})}$ & $\begin{array}{c}\text { Onsite } \\
\text { Receptor }^{(b)} \text { Full- } \\
\text { time Exposure }^{(\text {c) }}\end{array}$ & $\begin{array}{c}\text { Onsite }> \\
\text { Offsite }\end{array}$ & $\begin{array}{c}\text { Onsite Receptor }^{(b)} \\
\text { Working-Year }^{\left({ }^{(d)}\right.} \\
\text { Exposure }^{(2)}\end{array}$ & $\begin{array}{c}\text { Onsite > } \\
\text { Offsite }\end{array}$ \\
\hline $\mathrm{H}-3$ & $3.40 \mathrm{E}-05$ & $1.74 \mathrm{E}-05$ & & $8.25 \mathrm{E}-06$ & \\
\hline C-14 & $2.60 \mathrm{E}-03$ & 3.32E-04 & & $3.31 \mathrm{E}-04$ & \\
\hline $\mathrm{O}-15$ & $1.00 \mathrm{E}-09$ & $3.72 \mathrm{E}-07$ & $\checkmark$ & $8.49 \mathrm{E}-08$ & $\checkmark$ \\
\hline Ar-41 & $2.10 \mathrm{E}-05$ & $6.29 \mathrm{E}-05$ & $\checkmark$ & $1.57 \mathrm{E}-05$ & \\
\hline $\mathrm{Mn}-54$ & $2.40 \mathrm{E}-02$ & $6.34 \mathrm{E}-02$ & $\checkmark$ & $1.57 \mathrm{E}-02$ & \\
\hline Co-60 & $3.60 \mathrm{E}-01$ & $9.48 \mathrm{E}-01$ & $\checkmark$ & $2.35 \mathrm{E}-01$ & \\
\hline $\mathrm{Zn}-65$ & $3.60 \mathrm{E}-02$ & $3.59 \mathrm{E}-02$ & & $1.04 \mathrm{E}-02$ & \\
\hline $\mathrm{Zn}-69 \mathrm{~m}$ & $6.30 \mathrm{E}-05$ & $1.69 \mathrm{E}-04$ & $\checkmark$ & $4.31 \mathrm{E}-05$ & \\
\hline Kr-85 & $7.50 \mathrm{E}-08$ & $1.88 \mathrm{E}-07$ & $\checkmark$ & $5.25 \mathrm{E}-08$ & \\
\hline Sr-90 & $1.70 \mathrm{E}-01$ & $2.73 \mathrm{E}-02$ & & $1.29 \mathrm{E}-02$ & \\
\hline Zr-95 & $5.70 \mathrm{E}-03$ & $1.29 \mathrm{E}-02$ & $\checkmark$ & $3.23 \mathrm{E}-03$ & \\
\hline $\mathrm{Nb}-95$ & $7.00 \mathrm{E}-03$ & $7.50 \mathrm{E}-03$ & $\checkmark$ & $2.10 \mathrm{E}-03$ & \\
\hline Ru-103 & $2.70 \mathrm{E}-03$ & $5.61 \mathrm{E}-03$ & $\checkmark$ & $1.41 \mathrm{E}-03$ & \\
\hline $\mathrm{Ru}-106$ & $2.30 \mathrm{E}-02$ & $4.21 \mathrm{E}-02$ & $\checkmark$ & $1.12 \mathrm{E}-02$ & \\
\hline Ag-110 & $4.80 \mathrm{E}-26$ & $7.03 \mathrm{E}-16$ & $\checkmark$ & $1.60 \mathrm{E}-16$ & $\checkmark$ \\
\hline Sn-113 & $3.30 \mathrm{E}-03$ & $1.60 \mathrm{E}-03$ & & $5.62 \mathrm{E}-04$ & \\
\hline Sb-125 & $3.80 \mathrm{E}-02$ & $1.02 \mathrm{E}-01$ & $\checkmark$ & $2.52 \mathrm{E}-02$ & \\
\hline $\mathrm{I}-129$ & $6.20 \mathrm{E}-01$ & $2.21 \mathrm{E}-01$ & & 6.03E-02 & \\
\hline $\mathrm{I}-131$ & $2.00 \mathrm{E}-02$ & $4.94 \mathrm{E}-03$ & & $1.40 \mathrm{E}-03$ & \\
\hline Cs-134 & $1.50 \mathrm{E}-01$ & $2.81 \mathrm{E}-01$ & $\checkmark$ & $7.18 \mathrm{E}-02$ & \\
\hline Cs-137+D & $3.51 \mathrm{E}-01$ & $8.52 \mathrm{E}-01$ & $\checkmark$ & $2.12 \mathrm{E}-01$ & \\
\hline Cs-137 & $3.90 \mathrm{E}-02$ & $5.44 \mathrm{E}-03$ & & $3.44 \mathrm{E}-03$ & \\
\hline Ce-144 & $1.80 \mathrm{E}-02$ & $3.44 \mathrm{E}-02$ & $\checkmark$ & $9.15 \mathrm{E}-03$ & \\
\hline Pm-147 & $1.60 \mathrm{E}-03$ & $3.43 \mathrm{E}-03$ & $\checkmark$ & $9.13 \mathrm{E}-04$ & \\
\hline Eu-152 & $3.60 \mathrm{E}-01$ & $9.62 \mathrm{E}-01$ & $\checkmark$ & $2.38 \mathrm{E}-01$ & \\
\hline Eu-154 & $2.90 \mathrm{E}-01$ & $7.77 \mathrm{E}-01$ & $\checkmark$ & $1.92 \mathrm{E}-01$ & \\
\hline Eu-155 & $1.20 \mathrm{E}-02$ & $3.05 \mathrm{E}-02$ & $\checkmark$ & $7.60 \mathrm{E}-03$ & \\
\hline Tl-208 & $7.40 \mathrm{E}-08$ & $6.36 \mathrm{E}-06$ & $\checkmark$ & $1.45 \mathrm{E}-06$ & $\checkmark$ \\
\hline $\mathrm{Pb}-212$ & $5.10 \mathrm{E}-03$ & $1.45 \mathrm{E}-02$ & $\checkmark$ & $3.74 \mathrm{E}-03$ & \\
\hline Bi-212 & $6.30 \mathrm{E}-04$ & $2.29 \mathrm{E}-03$ & $\checkmark$ & $5.48 \mathrm{E}-04$ & \\
\hline U-234 & $4.50 \mathrm{E}+00$ & $1.16 \mathrm{E}+01$ & $\checkmark$ & $3.07 \mathrm{E}+00$ & \\
\hline U-235 & $4.30 \mathrm{E}+00$ & $1.11 \mathrm{E}+01$ & $\checkmark$ & $2.93 \mathrm{E}+00$ & \\
\hline U-238 & $4.10 \mathrm{E}+00$ & $1.03 \mathrm{E}+01$ & $\checkmark$ & $2.72 \mathrm{E}+00$ & \\
\hline $\mathrm{Pu}-239$ & $1.20 \mathrm{E}+01$ & $3.00 \mathrm{E}+01$ & $\checkmark$ & $7.93 \mathrm{E}+00$ & \\
\hline $\mathrm{Pu}-241$ & $1.90 \mathrm{E}-01$ & $4.56 \mathrm{E}-01$ & $\checkmark$ & $1.21 \mathrm{E}-01$ & \\
\hline $\mathrm{Am}-241$ & $1.90 \mathrm{E}+01$ & $4.76 \mathrm{E}+01$ & $\checkmark$ & $1.26 \mathrm{E}+01$ & \\
\hline \multicolumn{6}{|c|}{$\begin{array}{l}\text { (a) Offsite receptor at } 9100 \mathrm{~m} \text { SE (HNF-3602, Hill and Rittmann 1999). } \\
\text { (b) Onsite receptor at Energy NW, } 4.4 \mathrm{~km} \text { NNE. } \\
\text { (c) Assumes } 8766 \mathrm{~h} / \mathrm{y} \text { occupancy onsite for compliance with EPA regulations. Includes dose from ingestion } \\
\text { of food produced regionally. } \\
\text { (d) Assumes } 2000 \mathrm{~h} / \mathrm{y} \text { occupancy onsite for compliance with DOE standards. Includes dose from ingestion } \\
\text { of food produced regionally and exposure via inhalation and external pathways typical of an "average" } \\
\text { member of the population for remainder of year }(6766 \mathrm{~h} / \mathrm{y}) \text {. }\end{array}$} \\
\hline
\end{tabular}

\section{F.1 Reference}

Hill, J. S., and P. D. Rittmann. 1999. Volume 1: Calculating Potential to Emit Releases and Doses for FEMPs and NOCs. HNF-3602, Vol. 1, Fluor Daniel Hanford, Inc., Richland, Washington. 


\section{Appendix G}

Sportsman Dose 


\section{Appendix G}

\section{Sportsman Dose}

Wildlife has access to areas of the site that contain radioactive materials and some do become contaminated. Sometimes, contaminated wildlife travel off the site. Sampling is conducted on the site to estimate the maximum contamination levels that might possibly exist in animals hunted off the site. Because this scenario has a relatively low probability of occurring, these doses are not included in the maximally exposed individual calculation.

The highest dose, calculated using methodology set by Soldat et al. (1990), based on maximum radionuclide concentrations in game animals collected from 1993 through 1999, was determined to be 0.05 mrem from the consumption of a duck sample collected in 1993 (see Table G.1 and Figure G.1).

Table G.1. Radiological Dose from 1-kg Consumption, mrem

\begin{tabular}{|l|c|c|c|c|c|c|c|}
\hline Year & Deer & Waterfowl & Pheasant & Rabbit & Fish & Other & Maximum \\
\hline 1993 & 0.02 & $\begin{array}{c}0.05 \\
\text { (duck) }\end{array}$ & 0.001 & 0.02 & 0.001 & & Waterfowl \\
\hline 1994 & 0.0004 & & 0.008 & 0.002 & 0.007 & $\begin{array}{c}0.02 \\
\text { (clams) }\end{array}$ & Clams \\
\hline 1995 & 0.002 & $\begin{array}{c}0.0004 \\
\text { (goose) }\end{array}$ & & & 0.002 & & Deer/Fish \\
\hline 1996 & 0.001 & & 0.0002 & & 0.001 & & Deer/Fish \\
\hline 1997 & & $\begin{array}{c}0.004 \\
\text { (goose) }\end{array}$ & & & 0.002 & & Fish \\
\hline 1998 & 0.0003 & & 0.0009 & & 0.02 & & Fish \\
\hline 1999 & & $\begin{array}{c}0.002 \\
(\text { goose })\end{array}$ & & 0.003 & & & Rabbit \\
\hline
\end{tabular}

G.1 


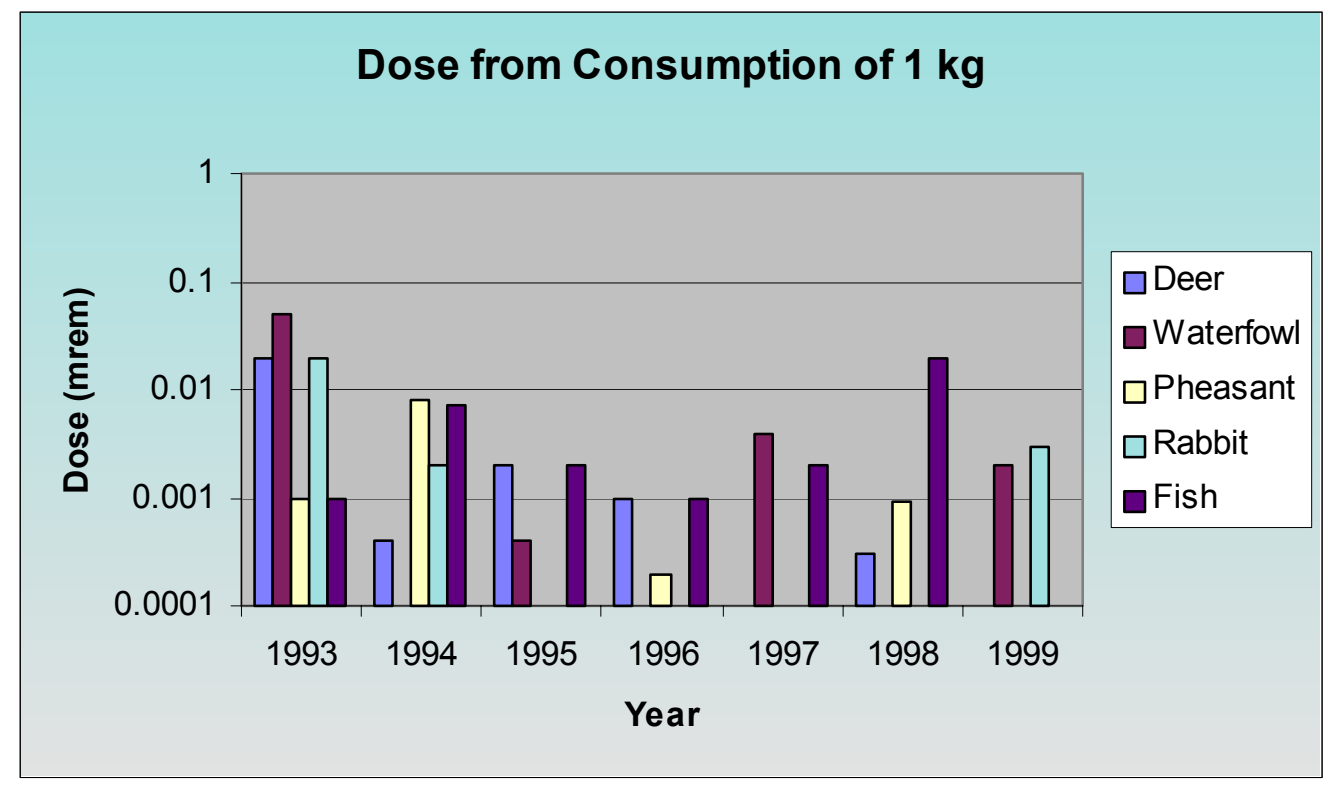

Figure G.1. Calculated Radiological Doses from 1-kg Consumption of Game Animals Taken from the Hanford Site 


\section{Distribution}

No. of

\section{Copies}

OFFSITE

R. S. Acelrod

Washington State Department of Health

Box 385

2839 W. Kennewick Ave.

Kennewick, WA 99336

C. Cannon

ATG, Inc.

P.O. Box 969

Richland, WA 99352

A. W. Conklin

Washington State Department of Health

Air Emissions and Defense Waste Section

Division of Radiation Protection

P.O. Box 47827

Olympia, WA 98504-7827

\section{A. T. Cooper}

Division of Radiation Protection

Washington State Dept. of Health

3107 W. 47th

Kennewick, WA 99337

A. Danielson

Washington State Dept. of Health

5508 Englewood Avenue

Yakima, WA 98908

\section{J. E. Erickson}

Washington State Department of Health

Air Emissions and Defense Waste Section

Division of Radiation Protection

P.O. Box 47827

Olympia, WA 98504-7827
No. of

\section{Copies}

R. Evans

WA Department of Health

Division of Radiation Protection

2839 W. Kennewick Ave,

Box 385

Kennewick, WA 99336

R. Gay, Grant Contracts Officer

Environmental Science and Technology

Program

Confederated Tribes of the Umatilla Indian

Reservation

P.O. Box 638

Pendleton, OR 97801

R. Jim, Manager

Environmental Restoration/Waste

Management Program

Yakama Nation

2808 Main Street

Union Gap, WA 98903

G. L. Laws

Washington State Department of Health

Box 385

2839 W. Kennewick Ave.

Kennewick, WA 99336

J. M. Leitch

U.S. Environmental Protection Agency

Region 10

1200 Sixth Avenue

Seattle WA 98101

Distr.1 
No. of

Copies

P. J. Martell

Washington State Department of Health

Box 385

2839 W. Kennewick Ave.

Kennewick, WA 99336

D. McBaugh, Head, MS-7827

Environmental Radiation Section

Division of Radiation Protection

Washington State Dept. of Health

P.O. Box 47827

Olympia, WA 98504-7827

L. Seelatsee

Wanapum

Grant County P.U.D.

30 "C" Street, S.W.

P.O. Box 878

Ephrata, WA 98823

J. W. Schmidt

Washington State Department of Health

Box 385

2839 W. Kennewick Ave.

Kennewick, WA 99336

P. Sobotta, Director

Environmental Restoration/Waste

Management Program

Nez Perce Tribe

P.O. Box 365

Lapwai, ID 83540

G. A. Vazquez

U.S. Department of Energy Headquarters

Forrestal Building, EH-412

1000 Independence Ave. SW

Washington, D.C. 20585

\section{ONSITE}

S. Van Verst

Division of Radiation Protection

Washington State Dept. of Health

P.O. Box 47827

Olympia, WA 98504-7827

Bechtel Hanford, Inc.

K. A. Gano

$\mathrm{H} 0-23$

C.J. Kemp

H0-19

J.G. Woolard

$\mathrm{H} 0-02$

CH2MHill Hanford Group, Inc.

G.M. Crummel

R1-51

J.J. Luke

R1-51

K.S. Tollefson

R1-51

DynCorp Tri-Cities Services, Inc.

C.J. Grando

G3-26

Duratek

J. J. Dorian

H1-11

Fluor Hanford

J.M. Barnett

L1-05

J.A. Bates

G1-30

W.E. Davis

G1-37

L.P. Diediker

G1-29

R.H. Engelmann

G1-30

M.T. Jansky

G1-30

A.R. Johnson

H5-26

D.J. Rokkan

G1-29

D.J. Watson

X3-79

K.R. Welsch

G1-30 
No. of

Copies

U.S. Department of Energy, Office of River

Protection

D.W. Bowser

G.M. Neath

H6-60

H6-60

U.S. Department of Energy, Richland Operations Office

L. M. Bowers

P.F.X. Dunigan

J. B. Hall

W. M. Glines

A.P. Larsen

D. C. Ward

S. H. Wisness

DOE Public Reading Room
No. of

Copies

$\underline{\text { Pacific Northwest National Laboratory }}$

E. J. Antonio

K3-54

P7-68

BSRC

P7-68

K6-75

P7-68

K6-75

P7-79

K3-54

K6-75

K6-75

K3-54

K3-54

K3-54

Sequim

P7-79 